\title{
High Order Harmonic Generation in Rare Gases
}

\author{
K. S. Budil
}

May 1, 1994

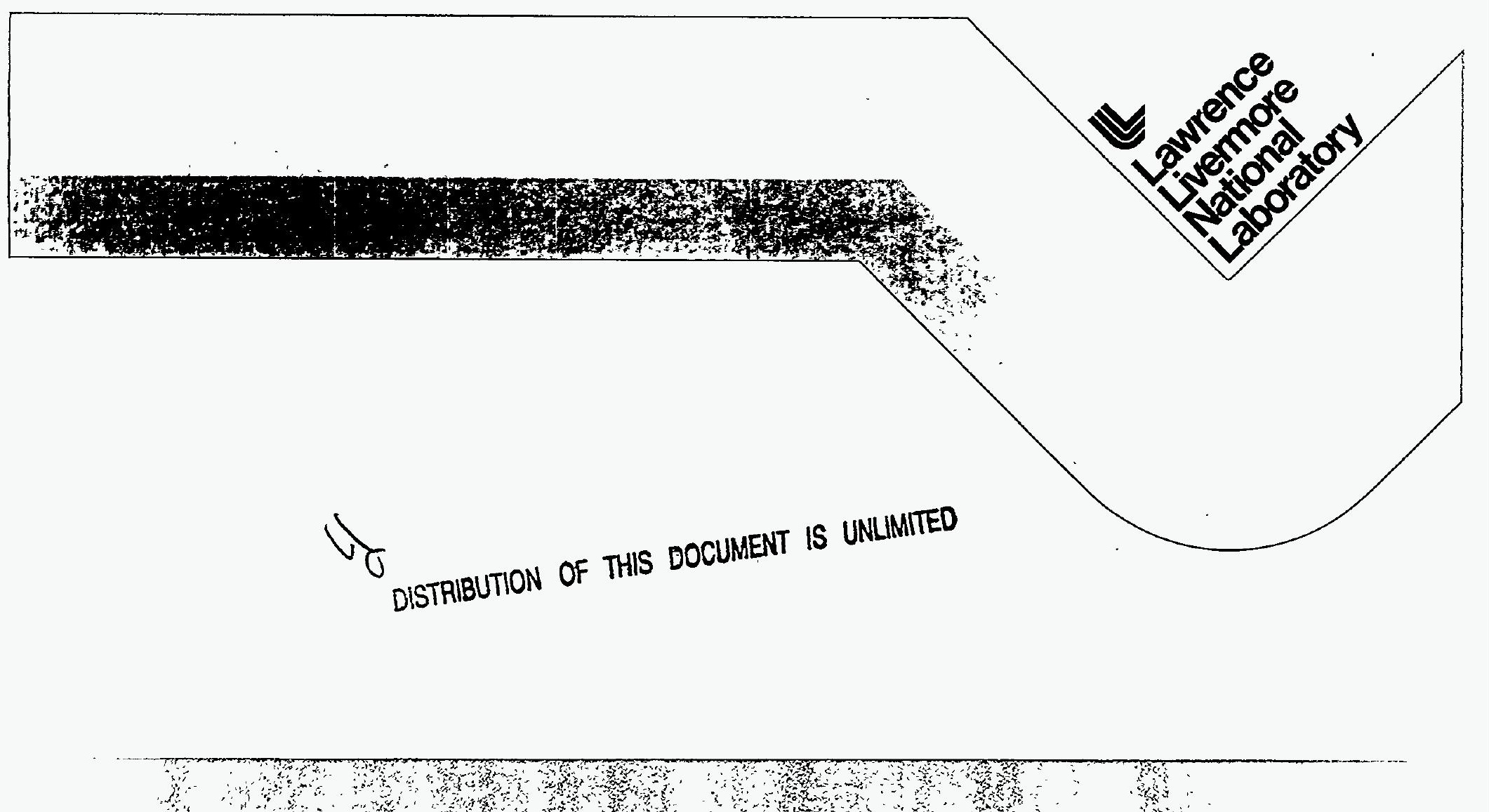




\section{DISCLAIMER}

This document was prepared as an account of work sponsored by an agency of the United States Government. Neither the United States Government nor the University of California nor any of their employees, makes any warranty, express or implied, or assumes any legal liability or responsibility for the accuracy, completeness, or usefulness of any information, apparatus, product, or process disclosed, or represents that its use would not infringe privately owned rights. Reference herein to any specific commercial product, process, or service by trade name, trademark, manufacturer, or otherwise, does not necessarily constitute or imply its endorsement, recommendation, or favoring by the United States Government or the University of California. The views and opinions of authors expressed herein do not necessarily state or reflect those of the United States Government or the University of California, and shall not be used for advertising or product endorsement purposes.

This report has been reproduced

directly from the best available copy.

Available to DOE and DOE contractors from the Office of Scientific and Technical Information P.O. Box 62, Oak Ridgie, TN 37831

Prices available from (615) 576-8401, FTS 626-8401

Available to the public from the

National Technical Infonmation Service

U.S. Department of Commerce

5285 Port Royal Rd.,

Springfield, VA 22161

Work performed under the auspices of the U.S. Department of En ergy by Lawrence Livermore National Laboratory under Contract W-7405-Eng-48. 


\section{DISCLAIMER}

Portions of this document may be illegible in electronic image products. Images are produced from the best available original document. 
UCRL-LR-118510

Distribution Category UC-700

\title{
High Order Harmonic Generation in Rare Gases
}

\author{
K. S. Budil
}

Manuscript date: May 1, 1994

LAWRENCE LIVERMORE NATIONAL LABORATORY University of California • Livermore, California • 94551 


\section{High Order Harmonic Generation in Rare Gases}

. By

Kimberly Susan Budil

BS. University of Illinois. 1.987

MS. Eniversity of California. Davis 1988

\section{DISSERTATION}

Submitted in partial satisfaction of the requirements for the degree of DOCTOR OF PHILOSOPHY

in

Applied Science

in the

- GRADUATE DIVISION

of the

ENIVERSITY OF CALIFORNIA

DAVIS

Approved:

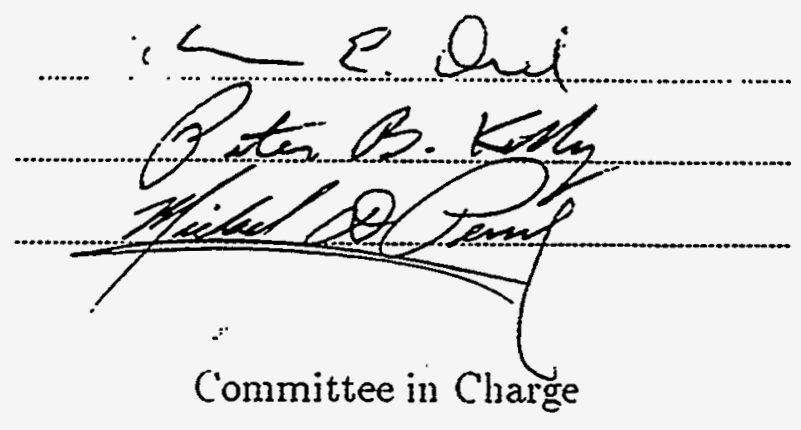

1994 


\section{•}

. 


\section{Acknowledgements}

It's hard to believe that I am actually finished and after six years there are many people to whom I owe a debt of gratitude for helping me get to this point. First, of course, must come the members of my thesis committee, Ann Orel-Woodin, Peter Kelly, and Mike Perry. Ann, in the dual capacity of thesis advisor and graduate advisor, was immensely helpful and supportive and taught me a great deal about atomic physics. Mike as my laboratory supervisor was the driving force behind the pursuit of harmonic generation and provided a world-class environment in which to do research for which I am very grateful. Things did not always go smoothly, but in the end we managed to pull it all together.

There were many people I worked with who were good friends as well. John Crane and Sarah Allendorf taught me a lot about doing experimental research and made it a lot of fun as well. Todd Ditmire always provided enthusiasm, ideas, heavy metal music, and an excuse to hoot. Hoang Nguyen and Steve Herman shared their laser expertise and were ever helpful. A long list of others helped out is ways to numerous to mention here but they are not forgotten.

I was given the distinct honor of working with (The) Donna Strickland who forever changed my view of science and myself. I have tried to emulate her life as best I can.

I owe a tremendous debt to Ken Schafer, without whom I would never have successfully navigated the treacherous waters of theory. He patiently endured my endless questions and problems and was a staunch ally when I needed one most. Ken Kulander was a great help as well, both for his immense expertise and his support. Ken and Ken, of course, bear no responsibility for my shortcomings as a theorist.

iii 
Special thanks are due to Anne L'Huillier, with whom the bulk of these experiments were conducted during her stay at Livermore. Without Anne's incredible knowledge of the field, her guidance, and particularly her confidence, most of this work would not have been possible. Her student, Pascal Salières was the other member of our team and I am grateful to him for helping to make this an enjoyable, as well as very successful, experience.

My friends and cohorts through these years cleserve special recognition. The Dr.'s Miller (Doug and Debbie) were like surrogate parents at times and provided constant support, companionship, home-cooked meals, scientific and computational expertise. many fine bottles of wine and I can never begin to express how much I appreciate them. Nick Gentile was a very good friend with whom I shared many fine meals and very bad movies. The man that gave us Cloven Hoof and Captain Fred will never be forgotten. Christine Coverdale and I swam countless laps together and spent countless hours griping about everything. Most of the homemade things that make my house feel like home are due to her. Dave Larson provided many kind words when I most needed to hear them as well as a really good attitude about life. Bob Tench was always a role model and taught me how to water ski too. I picked up many new hobbies from rock climbing (thanks to Dave Grote) to volleyball (thanks to Jeff Latkowski, Jay Hartley, and Brian Jones). There are many, many, many more people who have made this a great experience and they are all appreciated.

Dašenka and Alexandra, my "children", provided hours of entertainment and were always there for me when things were bad.

My family deserves some kind of award for enduring this experience. My brother Greg and my sister Jody were always there to listen and encourage, even when the end was very, very far away. My dad never gave up hope that I would reach the end some day and this is as much for him as for me. I only regret that my mother, who was always my biggest supporter, is not here to see this day though I am sure that somewhere she is smiling. This dissertation is dedicated to her memory. To all of my family I can only say that I love you and I could not have done this without you. 
Finally, I have to thank my husband, Dale Slone. While Dale came into the picture relatively late, I could never have finished this without his love and support. You're the best punk. 


\section{Contents}

Acknowledgements

iii

List of Tables

viii

List of Figures

ix

1 Introduction $\quad 3$

1.1 Description of the Problem . . . . . . . . . . . 7

1.1 .1 Perturbation Theory . . . . . . . . . . . . . 9

1.1 .2 Multipole Expansion . . . . . . . . . . . . . 13

1.1.3 Symmetry Properties . . . . . . . . . . . . . . . 16

1.1.4 Third Harmonic Generation . . . . . . . . . . 17

1.1 .5 Phase Matching . . . . . . . . . . . . . 18

1.1.6 "Weak" versus "Strong" Fields . . . . . . . . . . . 19

1.1.7 "Strong" Field Regime . . . . . . . . . . . . . 20

1.2 Research Program . . . . . . . . . . . . . . . . . 22

1.3 Plan of the Manuscript . . . . . . . . . . . . . 22

2 Experimental Facility 24

2.1 Short Pulse Dye Laser . . . . . . . . . . . . . . . . . 24

2.2 Cr:LiSAF Laser System $\ldots \ldots \ldots \ldots \ldots \ldots \ldots$

2.3 Experimental Apparatus . . . . . . . . . . . . . 43

vi 
3 Optical Harmonic Generation $\quad 49$

3.1 "Weak" Field Harmonic Generation . . . . . . . . . . . . . 49

3.2 Harmonic Generation in the "Strong" Field . . . . . . . . . 51

4 The Two-Step Semiclassical Model 60

4.1 Theoretical Approach . . . . . . . . . . . . . . 60

4.2 The Influence of Ellipticity $\ldots \ldots \ldots \ldots \ldots \ldots \ldots$

4.3 Simple Quantum Theory of Harmonic Generation . . . . . . . 78

4.3.1 Theoretical Approach . . . . . . . . . . . . . . . so

4.3.2 Model Calculations . . . . . . . . . . . . 82

5 The Harmonic "Source" $\quad 89$

5.1 Background and Motivation . . . . . . . . . . 89

5.2 Characterization of the Source . . . . . . . . . . . . . . 90

5.3 Application of High Order Harmonic Generation to Spectroscopy . . 98

6 Summary 105

6.1 Conclusions . . . . . . . . . . . . . . . 10.5

6.2 Future Work ..................... 106 


\section{List of Tables}

1.1 The current "bests" in the world of harmonic generation. . . . . . 6

2.1 Operating characteristics of the short pulse dye laser system. . . . . 39

4.1 Values of the Keldysh tunneling parameter for various experimental conditions. . . . . . . . . . . . . . . 70

4.2 Maximum number of photons produced for each harmonic order and generating medium presented herein. Intensity is presented in units of $\mathrm{W} / \mathrm{cm}^{2}$. For the $600 \mathrm{~nm}$ results, $f^{\#}=30$, and for the $825 \mathrm{~nm}$ results,

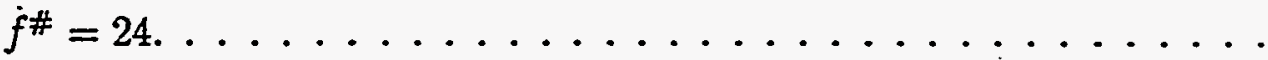




\section{List of Figures}

1.1 Schematic of the harmonic spectrum produced in neon by the LiSAF - laser at an intensity of $3.3 \times 10^{14} \mathrm{~W} / \mathrm{cm}^{2}$. The solid diamonds represent actual data points while the open circles are estimates of the number of photons produced for those harmonics which are outside the wavelength range of the spectrometer utilized. . . . . . . 5

1.2 Schematic of the process of harmonic generation. . . . . . . 7

2.1 Schematic of the short pulse high power dye laser system. (HWP $=$ half-wave plate, $\mathrm{QWP}=$ quarter-wave plate, $\mathrm{SHG}=$ second harmonic -generation, $\mathrm{PC}=$ Pockels cell, $\mathrm{TFP}, \mathrm{P}=$ thin film polarizer, $\mathrm{L}=$ lens, $\mathrm{SF}=$ spatial filter, $\mathrm{DL}=$ delay line, $\mathrm{DCS}=$ dichroic beam splitter, $\mathrm{PH}$ $=$ pinhole $\mathrm{A}=$ dye amplifier cuvette) $\ldots \ldots \ldots \ldots \ldots$

2.2 The temporal pulse profile of the mode-locked Nd:YAG oscillator. The secondary pulse is due to ringing in the fast photodiode. . . . . 26

2.3 Output power of the dye oscillator as a function of wavelength with rhodamine 6G in ethylene glycol as the gain medium. The oscillator is continuously tunable from $575-640 \mathrm{~nm}$. Extending this range requires changing the cavity optics as well as the dye utilized. . . . . . 27

2.4 Autocorrelation of dye oscillator pulses as a function of cavity length. 28

2.5 The measured single pass gain $G=E_{\text {out }} / E_{\text {in }}$ as a function of the pump energy in joules. The solid line is an exponential fit to the data. . . .

2.6 The energy level structure of $\mathrm{Nd}^{3+}$ with the $1.06 \mu \mathrm{m}$ lasing transition indicated. . . . . . . . . . . . . . 
2.7 The pulse amplitude in the regenerative amplifier as a function of the number of cavity round trips as calculated for the case of a constant initial population inversion and a gaussian input pulse. The pulse has reached its maximum amplitude after 9 round trips. . . . . . .

2.8 The experimentally observed pulse build up in the regenerative amplifier. The triggers to the Pockels cells are set $95 \mathrm{nsec}$ apart for maximum output, equivalent to 9 round trips. The pulse amplitude on the first several round trips is too small to be observed with the photodiode. .

2.9 The measured single pass gain $\mathrm{G}=\mathrm{E}_{\text {out }} / \mathrm{E}_{\text {in }}$ as a function of the pump energy in joules for the $9 \mathrm{~mm}$ amplifier head. The solid line is an exponential fit to the data. . . . . . . . . . . . 36

2.10 Schematic of the timing sequence for the regenerative amplifier. . . . 37

2.11 Single-shot autocorrelation of the amplified dye pulse. The solid curve is a calculation of the second order autocorrelation function for a hyperbolic secant squared pulse of $\tau=1.24 \mathrm{psec}$ width. The actual pulse

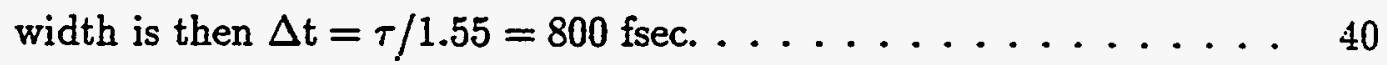

2.12 Schematic of the Cr:LiSAF laser system. ( $\mathrm{HR}=$ high reflector, $\mathrm{L}=$ lens, $\mathrm{TFP}=$ thin film polarizer, $\mathrm{A}=$ aperture, $\mathrm{HWP}=$ half-wave plate, $\mathrm{FR}=$ Faraday rotator $\ldots \ldots \ldots \ldots \ldots \ldots \ldots \ldots \ldots \ldots$

2.13 A block diagram of the experimental set-up required for optical harmonic generation. . . . . . . . . . . . . . . . 44

2.14 Experimental apparatus for the study of harmonic generation utilizing a .25 m Seya-Namioka monochromotor. . . . . . . . . . . 46

2.15 Experimental apparatus with $1 \mathrm{~m}$ flat-field monochromator. . . . . 47

3.1 Illustration of the problem of determing the field at point $P$ due to a collection of radiating oscillators. . . . . . . . . . 50

3.2 Third harmonic signal as a function of gas density for xenon at $~$ $10^{13} \mathrm{~W} / \mathrm{cm}^{2}$. The solid line shows an $N^{2}$ fit to the data. . . . . . 
3.3 Third harmonic intensity as a function of incident laser intensity. The solid line is a least squares fit to the data. . . . . . . . . 53

3.4 Harmonic spectrum produced in xenon with the dye laser operating at $600 \mathrm{~nm}$ as the driver. The intensity is $6 \times 10^{13} \mathrm{~W} / \mathrm{cm}^{2} \ldots \ldots \ldots 54$

3.5 Harmonic spectrum produced in xenon at $8 \times 10^{13} \mathrm{~W} / \mathrm{cm}^{2}$ with the LiSAF laser. . . . . . . . . . . . . . . . 5.5

3.6 Harmonic spectrum obtained in argon with the LiSAF laser. The intensity is $\sim 1.1 \times 10^{14} \mathrm{~W} / \mathrm{cm}^{2} \ldots \ldots \ldots \ldots \ldots$

3.7 Section of a harmonic spectrum obtained in neon at an intensity of $3.3 \times 10^{14} \mathrm{~W} / \mathrm{cm}^{2} . \ldots \ldots \ldots \ldots \ldots \ldots \ldots$

3.8 The relative number of photons at the tvrenty-fifth harmonic produced as a function of incident laser intensity for argon driven by the LiSAF laser. The solid lines are used to indicate the positions of changing slope of the curve. . . . . . . . . . . . . . . .

3.9 Relative number of harmonic photons produced as a function of the incident laser intensity for neon utilizing the LiSAF laser driver. . . 59

4.1 Schematic of the unperturbed potential for a hydrogen atom as well as the effective potential created by the influence of the laser field. The solid line within the potential well denotes the ground state wave function which can ionize by tunneling through the suppressed barrier.

4.2 Ionization rate of helium (thick curve) as a function of the phase of the incident laser field (thin curve). The peak intensity of the laser field is $5 \times 10^{14} \mathrm{~W} / \mathrm{cm}^{2} . \ldots \ldots \ldots \ldots \ldots \ldots$

4.3 Phase space plot of an electron trajectory for phase $\omega t_{o}=1.571$ radians. 64

4.4 The energy of the electron upon return to the nucleus as a function of the phase of the laser electric field at which the electron was born (a) without the influence of the atomic potential included and (b) with the influence of the atomic potential. ............ 
4.5 Energy histogram for uncorrected returns (solid line) and weighted returns with the $\omega^{-3}$. dependence of the oscillator strength included (open symbols) for two intensities. $I_{1}=2.5 \times 10^{14} \mathrm{~W} / \mathrm{cm}^{2}, U_{P_{1}}=$ $15.88 \mathrm{eV} ; \mathrm{I}_{2}=5.0 \times 10^{14} \mathrm{~W} / \mathrm{cm}^{2}, \mathrm{U}_{\mathrm{P} 2}=31.75 \mathrm{eV} . \ldots \ldots \ldots$ 66

4.6 An example of a trajectory generated for the case where the polarization of the incident laser has an ellipticity of .1. (a) Plot of $y$ vs $x$ and (b) plot of $r$ vs $t$ where the influence of the atomic potential has been included. . . . . . . . . . . . . . . .

4.7 Harmonics in xenon as a function of ellipticity. The curves represent the LOPT predictions for the decrease in harmonic intensity as the ellipticity of the incident beam is increased with decreasing profile width corresponding to increasing harmonic order. . . . . . . . .

4.8 Harmonics in neon as a function of ellipticity. The incident laser wavelength is $825 \mathrm{~nm}$ and the intensity is $\sim 1 \times 10^{15} \mathrm{~W} / \mathrm{cm}^{2} . \ldots \ldots$

4.9 Harmonics in argon as a function of ellipticity. The intensity is $3.5 \times$ $10^{14} \mathrm{~W} / \mathrm{cm}^{2}$ and $\lambda=825 \mathrm{~nm} . \ldots \ldots \ldots \ldots$

4.10 Harmonics in xenon as a function of ellipticity. The intensity is $1.2 \times$ $10^{14} \mathrm{~W} / \mathrm{cm}^{2} \ldots \ldots \ldots \ldots \ldots \ldots \ldots \ldots \ldots \ldots \ldots \ldots \ldots$

4.11 Harmonic spectrum obtained in neon at an intensity of $3.3 \times 10^{14} \mathrm{~W} / \mathrm{cm}^{2}$ with the Cr:LiSAF laser operating at $825 \mathrm{~nm} . \ldots \ldots \ldots 78$

4.12 The 17th, 19th, and 21st harmonics as a function of ellipticity for neon, argon, and xenon. The 21st harmonic was not observed in xenon. . . 79

4.13 Harmonic signal as a function of ellipticity for the 21st and 33rd harmonics produced in neon. The open symbols represent the experimental data from the previous section and the solid line is the model

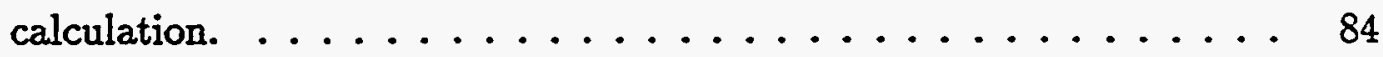

4.14 Harmonic signal as a function of ellipticity for the 43rd and 53rd harmonics produced in neon. The open symbols are experimental data and the solid line is the model calculation. . . . . . . . . . 
4.15 Harmonic signal as a function of ellipticity for the 19th and 21st harmonics in argon. The open symbols are experimental data and the solid line is the model calculation. . . . . . . . . . . . . .

4.16 Harmonic signal as a function of ellipticity for the 17th harmonic in xenon. The open symbols are experimental data and the solid line is the model calculation. . . . . . . . . . . . . . . .

5.1 The experimental apparatus for investigating the spatial profiles of high order harmonic radiation. . . . . . . . . . . . .

5.2 A contour plot of the 25th, 23rd and 21st harmonics produced in argon with the LiSAF laser at $1.2 \times 10^{14} \mathrm{~W} / \mathrm{cm}^{2} \ldots \ldots \ldots$

5.3 Integrated vertical lineouts for the 21st, 23rd, and 25th harmonics produced in argon with the LiSAF laser. The solid line is the experimentally measured spatial profile and the dotted line is a gaussian fit

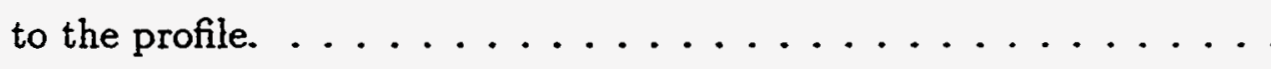

5.4 Spatial profiles of the 29th through 39 th harmonics produced in neon with the LiSAF laser at an intensity of $2.5 \times 10^{14} \mathrm{~W} / \mathrm{cm}^{2} \ldots \ldots$

5.5 Spatial profiles of the 29 th and 39 th harmonics produced in neon. The solid line is the experimental profiles and the dotted line is a gaussian fit to the data. . . . . . . . . . . . . . . . . .

5.6 Contour plot of the spatial profile of the 21st harmonic produced in argon with the LiSAF laser at an intensity of $8 \times 10^{14} \mathrm{~W} / \mathrm{cm}^{2}$. The field-free and blue-shifted positions of the harmonic are indicated. The contours labeled $\mathrm{a}, \mathrm{b}$, and $\mathrm{c}$ are of equal intensity. . . . . . . .

5.7 The experimental apparatus for utilizing high order harmonic radiation as a source to examine the photoionization cross section of the rare gases. 99

5.8 Number of ions produced in krypton as a function of number of harmonic photons produced by the seventh harmonic of the dye laser operating at $604.5 \mathrm{~nm}$. The solid line is a least squares fit to the data. . 101

5.9 The energy level structure of krypton. . . . . . . . . . . . . . 102 
5.10 Relative photoionization cross section of krypton as a function of photon energy. . . . . . . . . . . . . . . 103

5.11 Absolute photoionization cross section of neon as a function of photon energy. The solid line is the cross section as measured by a dipole $(e, e)$ spectrometer. . . . . . . . . . . . . . . . . 104 
Kimberly Susan Budil

April 1994

Applied Science

High Order Harmonic Generation

in Rare Gases

\begin{abstract}
$\underline{\text { Abstract }}$
The process of high order harmonic generation in atomic gases has shown great promise as a method of generating extremely short wavelength radiation, extending far into the extreme ultraviolet (XUV). The process is conceptually simple. A very intense laser pulse $\left(\mathrm{I} \sim 10^{13}-10^{14} \mathrm{~W} / \mathrm{cm}^{2}\right)$ is focused into a dense $(\sim$ $10^{17}$ particles $/ \mathrm{cm}^{3}$ ) atomic medium, causing the atoms to become polarized. These atomic dipoles are then coherently driven by the laser field and begin to radiate at odd harmonics of the laser field. This dissertation is a study of both the physical mechanism of harmonic generation as well as its development as a source of coherent XUV radiation.

Most previous theoretical efforts have been concerned with directly calculating the atomic dipole moment via numerical solution of the time-dependent Schrödinger equation or Floquet methods. Recently, a semiclassical theory has been proposed which provides a simple, intuitive description of harmonic generation. In this picture the process is treated in two steps. The atom ionizes via tunneling after which its classical motion in the laser field is studied. Electron trajectories which return to the vicinity of the nucleus may recombine and emit a harmonic photon, while those which do not return will ionize. An experiment was performed to test the validity of this model wherein the trajectory of the electron as it orbits the nucleus or ion core is perturbed by driving the process with elliptically, rather than linearly, polarized laser radiation. The semiclassical theory predicts a rapid turn-off of harmonic production
\end{abstract}


as the ellipticity of the driving field is increased. This decrease in harmonic production is observed experimentally and a simple quantum mechanical theory is used to model the data.

The second major focus of this work was on development of the harmonic "source". A series of experiments were performed examining the spatial profiles of the harmonics. The quality of the spatial profile is crucial if the harmonics are to be used as the source for experiments, particularly if they must be refocused. For intensities below the saturation intensity of the target species, smooth, structureless spatial profiles are observed with divergences consistent with those expected from a calculation of the far-field divergence angle of a gaussian beam. In the presence of intensities greater than the saturation intensity distortions are observed which are consistent with the interation of the driving laser with the plasma it is producing.

Finally, the harmonics were utilized as the source for a photoionization experiment. The photoionization cross section of neon was measured out to an incident photon energy of $103 \mathrm{eV}$ using a high power $\mathrm{Cr}$ : $\mathrm{LiSrAlF}_{6}$ (LiSAF) laser system as the driver and neon as the harmonic production medium. By utilizing a short pulse dye laser system as the driver and xenon as the harmonic production medium, autoionizing resonances in krypton were observed by tuning the seventh harmonic of the dye laser from 14.2 to $14.65 \mathrm{~nm}$. 


\section{Chapter 1}

\section{Introduction}

Since the early days of quantum mechanics the interaction of atoms with electromagnetic fields has been of great interest. As early as 1931, M. Goeppert-Mayer provided a theoretical description of the process of two-photon absorption [goppe31] although it would take many years before these processes could be studied experimentally. Since higher-order processes, nonlinear in nature, are generally much weaker than their linear counterparts, a source capable of providing very intense photon fluxes would be required to observe them. The advent of the laser brought the study of nonlinear optical processes from the theoretician's domain into the laboratory.

The experimental observation of second harmonic generation[frank61] as well as two-photon absorption [kaise61] revived interest in the study of higher order nonlinearities. Studies were confined to crystals, glasses, and liquids until Ward and New observed optical third harmonic generation in a gaseous medium by a focused laser beam in 1967.[ward69] Many theoretical efforts were directed at understanding this process [klein62, ward69, orr71] primarily focusing on perturbation theory. Since the incident electromagnetic field was much weaker than the Coulomb field binding the atom, the induced polarization could be expanded as a power series in the field. This allows an analytic solution for the intensity of the third harmonic radiation produced provided the susceptibility is known or can be calculated.

Since the dependence on the pump power is stronger for higher orders, there 
was speculation that conversion efficiencies to higher orders might exceed those to lower orders[reint78] with pump depletion by lower order processes or competing nonlinear processes thought to be the limiting factor. This was indeed seen to be the case for harmonic conversion in solids where high pump intensities led to dielectric breakdown and conversion efficiency decreased monotonically with increasing order. A low pressure gas was suggested as the optimal medium since the field strength required for breakdown is much greater. However, once again, many experiments observed saturation effects due to competing nonlinear processes.[reint84] Interest in extending this technique to very high orders waned although conversion to low orders became a common technique for generation of extreme ultraviolet radiation (XUV).

For the next decade, experimentalists continued to confirm that conversion efficiency fell off rapidly with increasing order although wavelengths as short as $35.5 \mathrm{~nm}$ were achieved.[bokor83] However, laser technology was also evolving and field strengths approaching the strength of the binding field of the atom were becoming achievable. In 1979, Agostini et al. [agost79] observed a new effect. While studying the photoelectron energy spectra produced by ionization of xenon with $1.064 \mu \mathrm{m}$ radiation at an intensity $\sim 10^{12} \mathrm{~W} / \mathrm{cm}^{2}$, a higher energy satellite peak was observed corresponding to the electron having absorbed more energy than required for ionization. This effect was subsequently named above-threshold ionization (ATI) and rapidly became an area of intense interest. Other groups undertook similar experiments and produced spectra showing many satellite peaks, each corresponding to the absorption of an additional photon by the atom before ionization.[kruit81]

These findings showed that in the presence of intense laser fields, higher order processes could occur with essentially the same probability as lower-order processes, a direct contradiction of the fundamental assumption of perturbation theory. The regime of nonperturbative processes had been entered. This suggested an interesting question with tremendous implications for harmonic generation. After absorbing many photons in excess of its binding energy could the electron radiatively recombine to the ground state instead of ionizing? A preliminary answer to this question was 
given in 1987 by an experiment performed by McPherson et al.[mcphe87] The group utilized a $\mathrm{KrF}$ excimer laser system $(248 \mathrm{~nm})$ focused to intensities in the range $10^{15}$ to $10^{16} \mathrm{~W} / \mathrm{cm}^{2}$ with neon as the generating medium. While conversion efficiency dropped off rapidly for orders five through eleven, the eleventh through seventeenth order were all produced with essentially the same efficiency, with seventeenth order corresponding to $14.6 \mathrm{~nm}$ radiation.[mcphe87] This work was rapidly followed by the observation of the thirty-third harmonic in argon and the twenty-first harmonic in xenon using a $1.064 \mu \mathrm{m}$ Nd:YAG laser.[ferra88, li89] To date harmonic production has been studied using numerous laser sources and target gases. The current "stateof-the-art" are summarized in Table 1.1.

In the presence of a very intense field, harmonic generation exhibits a characteristic spectrum shown in Figure 1.1. Conversion efficiency drops rapidly for the first few orders followed by a long plateau, or region of essentially constant conversion efficiency, ending in a cutoff determined by the laser intensity and wavelength.

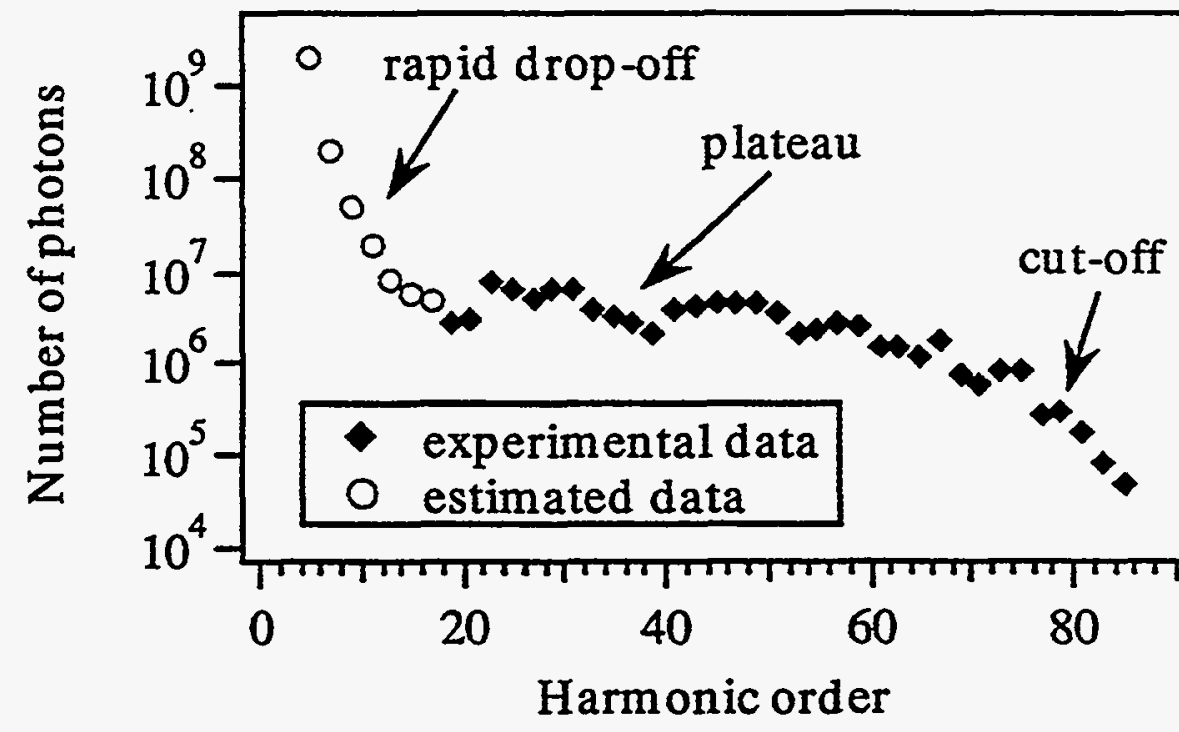

Figure 1.1: Schematic of the harmonic spectrum produced in neon by the LiSAF laser at an intensity of $3.3 \times 10^{14} \mathrm{~W} / \mathrm{cm}^{2}$. The solid diamonds represent actual data points while the open circles are estimates of the number of photons produced for those harmonics which are outside the wavelength range of the spectrometer utilized. 


\begin{tabular}{|c|c|c|c|c|c|}
\hline Group & laser & nonlinear medium & order & wavelength & reference \\
\hline Tokyo & $\mathrm{KrF}$ & neon & 25 & $9.8 \mathrm{~nm}$ & [saruk91] \\
\hline Tokyo & dye & helium & 41 & $15.0 \mathrm{~nm}$ & [miyaz92] \\
\hline Saclay & Nd:Glass & neon & 135 & $7.8 \mathrm{~nm}$ & [lhuil93] \\
\hline Lund & Ti:Sapphire & helium & 93 & $8.6 \mathrm{~nm}$ & [lhuil93b] \\
\hline $\begin{array}{l}\text { Imperial } \\
\text { College }\end{array}$ & Nd:Glass & helium & 119 & $8.9 \mathrm{~nm}$ & [tisch94] \\
\hline Stanford & Ti:Sapphire & neon & 109 & $7.4 \mathrm{~nm}$ & [mack193] \\
\hline \multirow[t]{3}{*}{ Livermore } & Nd:Glass $(2 \omega)$ & helium & 45 & $11.7 \mathrm{~nm}$ & [crane92] \\
\hline & Nd:Glass $(1 \omega)$ & helium & 143 & $7.0 \mathrm{~nm}$ & \multirow[t]{2}{*}{ [perry93] } \\
\hline & Li:SAF & neon & 83 & $9.9 \mathrm{~nm}$ & \\
\hline
\end{tabular}

Table 1.1: The current "bests" in the world of harmonic generation. 


\subsection{Description of the Problem}

Conceptually, the problem of harmonic generation in a gaseous medium is very simple. A laser beam is focused into the medium and polarizes the atoms contained within

\section{pulsed gas jet}

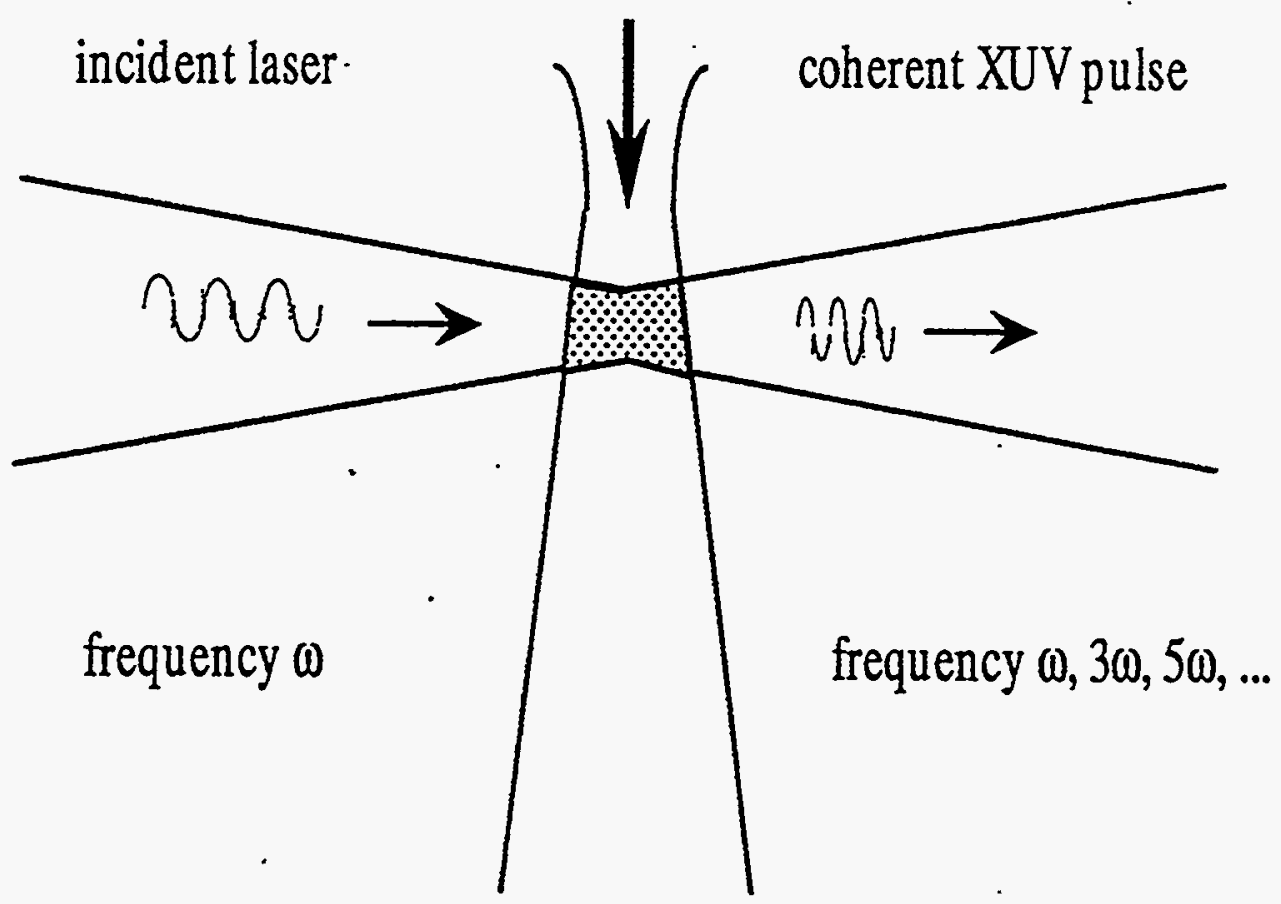

Figure 1.2: Schematic of the process of harmonic generation.

the focus. (Figure 1.2) These atomic dipoles continue to be driven in the field and. like a collection of driven harmonic oscillators, begin to radiate at odd harmonics of the incident field. Because of the symmetry of the atomic medium, the production of even harmonics is dipole forbidden. While this problem has been approached in many ways, the simplest approach is to begin with the case of a very weak incident laser field.

The amplitude of a harmonic wave generated by the interaction of an incident 
electric field with the polarized medium can be calculated using the Maxwell equations

$$
\begin{aligned}
\nabla \cdot \mathbf{D} & =4 \pi \rho \\
\nabla \times \mathbf{H} & =\frac{4 \pi}{c} \mathrm{~J}+\frac{1}{c} \frac{\partial \mathbf{D}}{\partial \mathrm{t}} \\
\nabla \times \mathbf{E} & =-\frac{1}{c} \frac{\partial \mathbf{B}}{\partial t} \\
\nabla \cdot \mathbf{B} & =0
\end{aligned}
$$

in Gaussian units. Provided the medium is not magnetized, the constitutive relations are given by

$$
\begin{aligned}
& \mathbf{H}=\mathbf{B} \\
& \mathbf{D}=\mathbf{E}+4 \pi \mathbf{P} .
\end{aligned}
$$

It is very straightforward to derive a wave equation for the electric field. First, the curl of equation 1.3 is taken yielding

$$
\nabla \times(\nabla \times \mathbf{E})=-\frac{1}{c} \nabla \times \frac{\partial \mathbf{B}}{\partial t} .
$$

Utilizing the vector identity $\nabla \times(\nabla \times \mathbf{V})=\nabla(\nabla \cdot \mathbf{V})-\nabla^{2} \mathbf{V}$, the left-hand side may be expanded to yield

$$
-\nabla^{2} \mathbf{E}+\nabla(\nabla \cdot \mathbf{E})=-\frac{1}{c} \nabla \times \frac{\partial \mathbf{B}}{\partial \mathrm{t}}
$$

Since the curl can be moved inside the time derivative on the right-hand side of equation 1.9 and there are no current densities present $(J=0)$, equations 1.5 and 1.2 may be substituted to give

$$
-\nabla^{2} \mathbf{E}+\nabla(\nabla \cdot \mathbf{E})=-\frac{1}{c} \frac{\partial}{\partial \mathrm{t}}\left[\frac{1}{c} \frac{\partial \mathrm{D}}{\partial \mathrm{t}}\right]
$$

Provided there are no free charges present, as is the case in a neutral atomic medium, $\rho=0$ and

$$
\nabla \cdot \mathbf{D}=\nabla \cdot \mathbf{E}+4 \pi \nabla \cdot \mathbf{P}=0
$$


Since the polarization $\mathrm{P}$ can be expressed as some function of the incident electric field, $P=\Psi(E)$, equation 1.10 can be written as

$$
(\nabla \cdot \mathbf{E})\left(1+4 \pi \Psi\left[\mathcal{O}\left(\mathrm{E}^{2}\right)\right]\right)=0
$$

Since the second term is small, this can be approximated as

$$
\nabla \cdot \mathbf{E}=\mathbf{0}
$$

Equation 1.9 becomes

$$
-\nabla^{2} \mathbf{E}=-\frac{1}{c} \frac{\partial}{\partial \mathrm{t}}\left[\frac{1}{c} \frac{\partial \mathrm{D}}{\partial \mathrm{t}}\right]
$$

Now equation 1.6 may be substituted to yield

$$
-\nabla^{2} \mathbf{E}=-\frac{1}{c^{2}} \frac{\partial^{2}(\mathbf{E}+4 \pi \mathbf{P})}{\partial \mathrm{t}^{2}}
$$

or

$$
\nabla^{2} \mathbf{E}-\frac{1}{c^{2}} \frac{\partial^{2} \mathbf{E}}{\partial t^{2}}=4 \pi \frac{\partial^{2} \mathbf{P}}{\partial t^{2}}
$$

\subsubsection{Perturbation Theory}

When the laser field is very weak compared to the field which binds the atom, it may be treated as a perturbation on the atomic potential and lowest-order perturbation theory (LOPT) may be applied to the problem. In the presence of weak pump fields, the polarization can be represented as a sum of linear and nonlinear contributions, $\mathbf{P}=\mathbf{P}^{\mathrm{L}}+\mathbf{P}^{\mathrm{NL}}$. The linear component may be expressed as $\mathbf{P}^{\mathrm{L}}(\mathbf{r}, \mathbf{t})=$ $\mathrm{N} \chi^{(1)}(\mathbf{k}, \omega) \mathbf{E}(\mathbf{r}, \mathrm{t})$ where $\chi^{(1)}$ is the linear susceptibility and $\mathrm{N}$ is the density of the atomic medium. This relationship between the electric field and the linear polarization will be valid as long as the pulse duration is long compared to the response time of the medium. Applying these to equation 1.15 yields

$$
\nabla^{2} \mathbf{E}-\frac{1}{c^{2}}\left(1+4 \pi N \chi^{(1)}\right) \frac{\partial^{2} \mathbf{E}}{\partial t^{2}}=\frac{4 \pi}{c^{2}} \frac{\partial^{2} \mathbf{P}^{N L}}{\partial t^{2}}
$$

For most laser radiation, it is reasonable to assume that the field can be represented as the product of a rapidly oscilliting term with an amplitude which oscillates much 
more slowly in time. Introducing this slowly-varying envelope approximation, the electric field may be expressed as

$$
E=\frac{1}{2}\left(A(r, z, t) e^{-i(k \cdot r-\omega t)}+\text { c.c. }\right)
$$

or

$$
\mathrm{E}=\frac{1}{2}\left(\mathrm{~A}(\mathrm{r}, \mathrm{z}, \mathrm{t}) e^{-i\left(\mathrm{k}_{z} z-\omega t\right)}+\text { c.c. }\right) .
$$

if the field is assumed to be propagating in the $z$-direction. When more than one field is present, the total field is given by the sum over all of the fields

$$
E=\frac{1}{2} \sum_{i}\left[A_{i}(r, z, t) e^{-i\left(\omega_{i} t-k_{i} z\right)}+c . c .\right]
$$

The polarization can be expressed in a similar manner as

$$
\mathrm{P}=\frac{1}{2} \sum_{j}\left[\mathbf{P}_{j}(\mathrm{r}, z, \mathrm{t}) e^{-\mathrm{z}\left(\omega, \mathrm{t}-\mathrm{k}_{j}^{\mathrm{P} z} \mathrm{z}\right)}+\text { c.c. }\right]
$$

where $k_{j}^{P} \neq k_{j}$ in general.

Applying the slowly-varying envelope approximation means that the variation of the field amplitudes will be small in one optical period

$$
\begin{aligned}
& \frac{\partial^{2} \mathrm{~A}_{i}}{\partial \mathrm{t}^{2}} \ll \omega_{i} \frac{\partial \mathrm{A}_{i}}{\partial \mathrm{t}} \\
& \frac{\partial^{2} \mathrm{P}_{j}}{\partial \mathrm{t}^{2}} \ll \omega_{j} \frac{\partial \mathrm{F}_{j}}{\partial \mathrm{t}}
\end{aligned}
$$

or one wavelength

$$
\frac{\partial^{2} A_{i}}{\partial z^{2}} \ll k_{i} \frac{\partial A_{-i}}{\partial z}
$$

and the second-order derivatives may be neglected. Extending this approximation one step further in the case of the nonlinear polarization implies that

$$
\omega_{j} \frac{\partial \mathrm{P}_{j}}{\partial t} \ll \omega_{j}^{2} \mathrm{P}_{j}
$$

and equation 1.16 becomes

$$
\nabla_{\perp}^{2} \mathrm{~A}_{i}+2 i \mathrm{k}_{i} \frac{\partial \mathrm{A}_{i}}{\partial \mathrm{z}}-\frac{2 i \mathrm{n}_{i}^{2} \omega_{i}}{\mathrm{c}^{2}} \frac{\partial \mathrm{A}_{i}}{\partial \mathrm{t}}=\frac{-4 \pi \omega_{i}^{2}}{\mathrm{c}^{2}} \mathrm{P}_{i}^{\mathrm{NL}} e^{-i \Delta \mathrm{k}_{i 2}}
$$


Equation 1.25 was derived assuming that the susceptibility was dispersionless, $\chi^{(i)} \neq \chi^{(i)}(\omega)$, however for a small but finite frequency spread in the field, this dispersion must be accounted for. The different frequency components will propagate through the medium at different phase velocities leading to a tendency for the original coherence of the pulse to be lost and its shape distorted. For the case where the wave number spectrum is not too broad and dissipative effects are ignored an approximate solution can be found. If the wave number spectrum is peaked about some value $k_{o}$, the frequency can be expanded about that value as

$$
\omega(k)=\omega_{0}+\frac{d \omega}{d k} l_{0}\left(k-k_{0}\right)+\cdots
$$

The amplitude of the pulse in momentum space can be Fourier transformed using this expression,

$$
\begin{aligned}
u(\mathrm{x}, \mathrm{t}) & =\frac{1}{(2 \pi)^{1 / 2}} \int_{-\infty}^{\infty} A(k) e^{i(k x-\omega(k) t)} d k \\
& \simeq \frac{e^{i\left[\left.k_{0}(d \omega / d k)\right|_{0}-\omega_{0}\right] t}}{(2 \pi)^{1 / 2}} \int_{-\infty}^{\infty} A(k) e^{i\left[x-\left.(d \omega / d k)\right|_{0} t\right] k} d k \\
& \simeq u\left(\mathrm{x}-\left.\frac{d \omega}{d k}\right|_{0} t, 0\right) e^{i\left[\left.k_{0}(d \omega / d k)\right|_{0}-\omega_{0}\right] t}
\end{aligned}
$$

Thus an additional phase term is present and the pulse is undistorted in shape and travels with a velocity called the group velocity

$$
v_{\mathrm{g}}=\left.\frac{d \omega}{d k}\right|_{0}
$$

The frequency $\omega$ is related to $k$ through the dispersion relation

$$
k=\frac{\mathrm{n}(\omega) \omega}{c}
$$

where $n(\omega)$ is the refractive index of the medium. Now,

$$
\frac{d k}{d \omega}=\frac{\mathrm{n}(\omega)}{c}+\frac{\omega}{c} \frac{d \mathrm{n}}{d \omega}
$$

which may be substituted in equation 1.28 to give

$$
\mathrm{v}_{\mathrm{g}}=\frac{d \omega}{d k}
$$




$$
\begin{aligned}
& =\frac{c}{\mathrm{n}(\omega)+\omega(d \mathrm{n} / d \omega)} \\
& =\frac{c / \mathrm{n}(\omega)}{1+[\omega / \mathrm{n}(\omega)][d \mathrm{n}(\omega) / d \omega]} \\
& =\frac{\mathrm{v}_{\mathrm{ph}}}{1+[\omega / \mathrm{n}(\omega)][d \mathrm{n}(\omega) / d \omega]}
\end{aligned}
$$

This expression for the group velocity, $v_{g}$, can now be substituted for the phase velocity $v_{p}=(n(\omega) / c)$ in equation 1.25 to account for dispersion of the various frequency components.

A transformation can now be made to a coordinate system that propagates at the group velocity $v_{g}$,

$$
\begin{aligned}
& \mathrm{z}^{\prime}=\mathrm{z} \\
& \mathrm{t}^{\prime}=\mathrm{t}-\frac{\mathrm{z}}{\mathrm{v}_{\mathrm{g}}} .
\end{aligned}
$$

Now the partial with respect to $z^{\prime}$ is given by

$$
\begin{aligned}
\frac{\partial}{\partial z^{\prime}} & =\frac{\partial z}{\partial z^{\prime}} \frac{\partial}{\partial z}+\frac{\partial t}{\partial z^{\prime}} \frac{\partial}{\partial t} \\
& =\frac{\partial}{\partial z}+\frac{\partial\left(t^{\prime}+z / v_{g}\right)}{\partial z^{\prime}} \frac{\partial}{\partial t} \\
& =\frac{\partial}{\partial z}+\left[\frac{\partial t}{\partial z^{\prime}}+\frac{1}{v_{g}} \frac{\partial z}{\partial z^{\prime}}\right] \frac{\partial}{\partial t} \\
& =\frac{\partial}{\partial z}+\frac{1}{v_{g}} \frac{\partial}{\partial t}
\end{aligned}
$$

and equation 1.25 becomes

$$
\nabla_{\perp}^{2} A_{i}\left(r, z^{\prime} t^{\prime}\right)+2 i k_{i} \frac{\partial A_{i}\left(r, z^{\prime} t^{\prime}\right)}{\partial z^{\prime}}=\frac{-4 \pi \omega_{i}^{2}}{c^{2}} P_{i}^{N L} e^{-i \Delta k_{i} z} .
$$

In general, any problem can be solved in this fashion provided the exact form of the polarization is known. Since this is typically not the case, further approximations will be required. 


\subsubsection{Multipole Expansion}

One convenient method is to make a multipole expansion of the polarization in terms of the moments of the electric field [jacks75]

$$
\mathbf{P}(\mathrm{r}, \mathrm{z}, \mathrm{t})=\mathbf{P}_{\mathrm{d}}(\mathrm{r}, \mathrm{z}, \mathrm{t})+\nabla \mathrm{Q}(\mathrm{r}, \mathrm{z}, \mathrm{t})+\nabla: \nabla O(\mathrm{r}, \mathrm{z}, \mathrm{t})+\cdots
$$

where the first term is the dipole contribution, the second the quadrupole, the third the octupole and so on. In the case of multipole radiation in atoms, the total power radiated by an electric multipole of order $(\ell, m)$ is given by

$$
P_{\mathrm{E}}(\ell, m)=\frac{2 \pi c}{\left[(2 \ell+1]^{2}\right.}\left(\frac{\ell+1}{\ell}\right) k^{2 \ell+2}\left|Q_{\ell m}+Q_{\ell m}^{\prime}\right|^{2}
$$

where $k=\omega^{2} / c^{2}, Q_{\ell m}$ is the moment due to the oscillating charge density, and $Q_{\ell m}^{\prime}$ is due to the magnetization. The relative strengths of the multipoles can be examined via the radiative transition probability or reciprocal mean lifetime given by

$$
\frac{1}{\tau}=\frac{P}{\hbar \omega}
$$

The charge density is assumed to be $\rho(x)=\left(3 e / a^{3}\right) Y_{\ell m}(\theta, \phi)$ for $r<a$ and 0 otherwise. With this the moments can be estimated to be

$$
\begin{aligned}
& Q_{\ell m} \simeq \frac{3}{\ell+3} e a^{\ell} \\
& Q_{\ell m}^{\prime} \simeq g\left(\frac{\hbar \omega}{m c^{2}}\right) Q_{\ell m}
\end{aligned}
$$

where $g$ is the effective $g$ factor for the magnetic moments of the particles in the atomic system. Since the rest energies of the particles involved are always much greater than the radiative transition energies in atoms, $m c^{2} \gg \hbar \omega, Q_{\ell m}^{\prime}$ will always be very small relative to $Q_{\ell m}$. Substituting this result into equation 1.39 gives

$$
\frac{1}{\tau(\ell)}=\left(\frac{e^{2}}{\hbar c}\right) \frac{2 \pi}{\left[(2 \ell+1]^{2}\right.}\left(\frac{\ell+1}{\ell}\right)\left(\frac{3}{\ell+3}\right)^{2}(k \mathrm{a})^{2 \ell} \omega .
$$

Thus, the ratio of transition probabilities for successive orders of electric multipoles of the same frequency is

$$
\frac{[\tau(\ell+1)]^{-1}}{[\tau(\ell)]^{-1}} \sim \frac{(k \mathrm{a})^{2}}{4 \ell^{2}}
$$


keeping only terms of relative order $\left(1 / \ell^{2}\right)$. For an atomic system, $a \sim a_{0} / Z_{\text {eff }}$, where $a_{o}$ is the Bohr radius and $Z_{\text {eff }}$ is the effective nuclear charge. An estimate for $k a$ can be made by noting that the transition energy is typically of the order of

$$
\hbar \omega<\mathrm{Z}_{\text {eff }}^{2} \mathrm{Ry}=\mathrm{Z}_{\text {eff }}{ }^{2} \frac{e^{2}}{2 \mathrm{a}_{\mathrm{o}}}
$$

which yields,

$$
\begin{aligned}
\hbar k c & <\mathrm{Z}_{\text {eff }} \frac{e^{2}}{\mathrm{a}_{o}} \\
\frac{k \mathrm{a}_{\circ}}{\mathrm{Z}_{\mathrm{eff}}} & <\mathrm{Z}_{\text {eff }} \frac{e^{2}}{\hbar c} \\
\dot{k \mathrm{a}} & <\frac{\mathrm{Z}_{\text {eff }}}{137}
\end{aligned}
$$

so the strength of successive multipole transitions will decrease by a factor of $\left(\mathrm{Z}_{\mathrm{eff}} / 137\right)^{2}$. For a transition by a valence electron, as will be the case in harmonic generation, $\mathrm{Z}_{\text {eff }} \sim 1$, and the dipole transition will dominate the process. In the case of an X-ray transition, $\mathrm{Z}_{\mathrm{eff}} \leq \mathrm{Z}$ and higher order multipoles can become important.

Applying the dipole approximation, the total polarization in frequency space can be written as

$$
\mathbf{P}_{\mathbf{T}}(\mathbf{k}, \omega)=\mathbf{P}^{(1)}+\mathbf{P}^{(2)}(\mathbf{k}, \omega)+\mathbf{P}^{(3)}(\mathbf{k}, \omega)+\cdots
$$

where

$$
\begin{aligned}
& \mathbf{P}^{(1)}(\mathbf{k}, \omega)=\chi^{(1)}(\mathbf{k}, \omega) \cdot \mathbf{E}(\mathbf{k}, \omega) \\
& \mathbf{P}^{(2)}(\mathbf{k}, \omega)=\chi^{(2)}\left(\mathbf{k}=\mathbf{k}_{i}+\mathbf{k}_{j}, \omega=\omega_{i}+\omega_{j}\right): \mathrm{E}\left(\mathbf{k}_{i}, \omega_{i}\right) \mathbf{E}\left(\mathbf{k}_{j}, \omega_{j}\right) \\
& \dot{P}^{(3)}(\mathbf{k}, \omega)=\chi^{(3)}\left(\mathbf{k}=\mathbf{k}_{i}+\mathrm{k}_{j}+\mathrm{k}_{l}, \omega=\omega_{i}+\omega_{j}+\omega_{l}\right): \mathbf{E}\left(\mathbf{k}_{i}, \omega_{i}\right) \mathrm{E}\left(\mathrm{k}_{j}, \omega_{j}\right) \mathbf{E}\left(\mathrm{k}_{l}, \omega_{l}\right)
\end{aligned}
$$

where $\chi^{(n)}$ is the nth order susceptibility. Since the form of the polarization in the time domain is required, these expressions must be Fourier transformed which results in a convolution of the susceptibilities and the fields as

$$
\mathbf{P}^{(1)}(\mathbf{r}, \mathrm{t})=\int_{-\infty}^{\infty} \chi^{(1)}(\mathbf{k}, \omega) \cdot \mathbf{E}(\mathbf{k}, \omega) e^{-i \omega t} d \omega
$$


$\mathbf{P}^{(2)}(\mathbf{r}, \mathrm{t})=\int_{-\infty}^{\infty} \int_{-\infty}^{\infty} \chi^{(2)}\left(\mathbf{k}=\mathrm{k}_{i}+\mathrm{k}_{j}, \omega=\omega_{i}+\omega_{j}\right): \mathbf{E}\left(\mathbf{k}_{i}, \omega_{i}\right) \mathbf{E}\left(\mathbf{k}_{j}, \omega_{j}\right) e^{-i\left(\omega_{\mathrm{t}}+\omega_{j}\right) t} d \omega_{i} d \omega_{j}$

In the absence of resonances, the susceptibilities may be treated as constants and the integrals evaluated giving

$$
\begin{aligned}
\mathbf{P}^{(1)}(\mathbf{r}, \mathrm{t}) & =\chi^{(1)}(\mathbf{k}, \omega) \int \mathbf{E}(\mathbf{k}, \omega) e^{-i \omega t} d \omega=\chi^{(1)}(\mathbf{k}, \omega) \mathbf{E}(\mathbf{r}, \mathrm{t}) \\
\mathbf{P}^{(2)}(\mathbf{r}, t) & =\chi^{(2)}(\mathbf{k}, \omega): \int_{-\infty}^{\infty} \int_{-\infty}^{\infty} \mathbf{E}\left(\mathbf{k}_{i}, \omega_{i}\right) \mathbf{E}\left(\mathbf{k}_{j}, \omega_{j}\right) e^{-i\left(\omega_{i}+\omega_{j}\right) t} e^{\left.-i\left(\omega_{i}+\omega_{j}\right) t\right)} d \omega_{i} d \omega_{j} \\
& =\chi^{(2)}(\mathbf{k}, \omega): \mathbf{E}_{1}(\mathbf{r}, \mathrm{t}) \mathbf{E}_{2}(\mathbf{r}, \mathrm{t}) \\
\vdots &
\end{aligned}
$$

Thus the total polarization may be expressed in terms of the total electric field as

$$
\begin{aligned}
\mathbf{P}_{\mathbf{T}}(\mathbf{r}, \mathrm{t}) & =\mathbf{P}_{\mathbf{L}}(\mathbf{r}, \mathrm{t})+\mathbf{P}_{\mathrm{NL}}(\mathbf{r}, \mathrm{t}) \\
& =\chi^{(1)} \mathbf{E}_{\mathbf{T}}(\mathbf{r}, \mathrm{t})+\chi^{(2)} \mathbf{E}_{\mathbf{T}}{ }^{2}(\mathbf{r}, \mathrm{t})+\chi^{(3)} \mathbf{E}_{\mathbf{T}}{ }^{3}(\mathbf{r}, \mathrm{t})+\cdots
\end{aligned}
$$

where $E_{\mathbf{T}}(\mathbf{r}, \mathrm{t})=\mathbf{E}_{1}(\mathbf{r}, \mathrm{t})+\mathbf{E}_{2}(\mathbf{r}, \mathrm{t})+\cdots+\mathbf{E}_{\mathbf{N}}(\mathbf{r}, \mathrm{t})$.

The first-order susceptibility $\chi^{(1)}$ describes linear polarizations at frequency $\omega$ arising from fields at the same frequency. The nonlinear susceptibilities connect polarizations at frequency $\omega$ to fields at the constituent frequencies. For example, in sum-frequency generation the polarization at $\omega_{3}=\omega_{1}+\omega_{2}$ can arise from the secondorder term via fields at frequencies $\omega_{1}$ and $\omega_{2}$. We can proceed to express the nonlinear polarization in terms of the various field components,

$$
\mathrm{P}^{\mathrm{NL}}\left(\omega_{\mathrm{q}}\right)=g \mathrm{~N} \chi^{(\mathrm{q})} \mathrm{E}_{1} \cdots \mathrm{E}_{\mathrm{n}}
$$

where $g$ is a degeneracy factor corresponding to the number of distinct permutations of the fields. For the example of sum-frequency generation, the total field is

$$
E_{T}(t)=E_{1}(t)+E_{2}(t)
$$

and the total second-order term is given by

$$
\mathbf{P}^{(2)}(t)=\chi^{(2)}\left(\mathbf{E}_{1}(\mathrm{t})+\mathbf{E}_{2}(\mathrm{t})\right)^{2}
$$




$$
\begin{aligned}
& =\chi^{(2)}\left(\mathrm{E}_{1}^{2}+\mathrm{E}_{2}^{2}+2 \mathrm{E}_{1} \mathrm{E}_{2}\right) \\
& =\chi^{(2)}\left(-2 \omega_{1}, \omega_{1}, \omega_{1}\right) \mathrm{E}_{1}{ }^{2}+\chi^{(2)}\left(-2 \omega_{2}, \omega_{2}, \omega_{2}\right) \mathrm{E}_{2}{ }^{2}+2 \chi^{(2)}\left(-\omega_{3}, \omega_{1}, \omega_{2}\right) \mathrm{E}_{1} \mathrm{E}_{2}
\end{aligned}
$$

where the first two terms correspond to second harmonic generation by each of the incident fields with no degeneracy since they each arise from a single field and the third term corresponds to generation of the sum frequency $\omega_{3}$ and the degeneracy factor is 2. Similarly for a four-wave mixing process $\left(\omega_{4}=\omega_{1}+\omega_{2}+\omega_{3}\right)$, the degeneracies would be 1 (third harmonic generation of each frequency), 3 (sum frequency generation of $\omega_{4}=2 \omega_{i}+\omega_{j}$ ) and 6 (sum frequency generation of $\omega_{4}=\omega_{1}+\omega_{2}+\omega+3$ ) respectively. Additionally, equation 1.51 may be expressed in terms of the amplitudes of the field components as

$$
\mathrm{P}^{\mathrm{NL}}\left(\omega_{\mathrm{q}}\right)=\frac{\mathrm{gN}}{2^{\mathrm{n}-1}} \chi^{(\mathrm{q})} \mathrm{A}_{1} \cdots \mathrm{A}_{\mathrm{n}}
$$

where the factor of $2^{\mathrm{n}-1}$ arises from the $\frac{1}{2}$ in the definition of the field amplitudes.

\subsubsection{Symmetry Properties}

As described earlier, harmonic generation in an isotropic atomic medium gives rise to only odd harmonics of the incident field. This is a direct result of the symmetry properties of the medium and can be readily shown by examining the properties of the nonlinear susceptibilities. Consider a medium with a center of inversion symmetry with the second-order polarization having the form

$$
\mathrm{P}^{(2)}=\chi^{(2)} \mathrm{E}^{2} \text {. }
$$

If the coordinate system is now inverted, both $\mathrm{P}$ and $\mathrm{E}$ as vectors are odd with respect to coordinate inversion and therefore must change sign yielding

$$
\begin{aligned}
\mathrm{P}^{(2)} & =\chi^{(2) \prime}\left(\mathrm{E}^{\prime}\right)^{2} \\
-\mathrm{P}^{(2)} & =\chi^{(2) \prime}(-E)^{2} \\
-\mathrm{P}^{(2)} & =\chi^{(2) \prime} \mathrm{E}^{2} .
\end{aligned}
$$


However $\chi^{(2)}$ is invariate under inversion for an isotropic medium so equation 1.56 becomes

$$
\mathrm{P}^{(2)}=-\chi^{(2)} \mathrm{E}^{2} .
$$

Equations 1.55 and 1.56 will only be compatible if $\chi^{(2)}=0$. A similar result can be arrived at for each of the even-ordered susceptibilities in a medium with inversion symmetry, thus precluding the production of even harmonics. While this is strictly true only for the dipole susceptibility, under typical experimental conditions this term is dominant.

A quantum mechanical picture can also be used to understand the absence of even-ordered processes. For a photon to be emitted via a dipole transition a change in angular momentum of $\ell=1$ must occur. If an even number of linearly polarized photons are absorbed, each carrying an angular momentum of $\ell= \pm 1$, the total angular momentum of the final virtual state will be either 0 or a multiple of 2 . Thus, the state cannot emit a single photon back to the ground state in a dipole transition and conserve angular momentum. In the case of circularly polarized light, all of the photons will carry angular momentum of either + or -1 , so the magnitude of the total angular momentum of the virtual state will always be greater than 1 and a single photon transition will be dipole forbidden.

\subsubsection{Third Harmonic Generation}

One readily solved case is that of third harmonic generation of a plane wave, $A_{i}(r, z)=$ $A_{i}(z)$. The coupled equations for the $1 \omega$ and $3 \omega$ fields are then

$$
2 i k_{1} \frac{\partial A_{1}}{\partial z}=\frac{3 \pi \omega_{1}^{2}}{c^{2}} \mathrm{~N} \chi^{(3)}\left(-\omega_{1}, 3 \omega_{1},-\omega_{1},-\omega_{1}\right) \mathrm{A}_{3} \mathrm{~A}_{1}^{* 2} e^{-i \Delta k z}
$$

and

$$
2 i k_{3} \frac{\partial \mathrm{A}_{3}}{\partial z}=\frac{-\pi \omega_{3}^{2}}{c^{2}} \mathrm{~N} \chi^{(3)}\left(-3 \omega_{1}, \omega_{1}, \omega_{1}, \omega_{1}\right) \mathrm{A}_{1}^{3} e^{-i \Delta k z} .
$$

If reconversion to $1 \omega$ is neglected as is the case for weak pump fields this reduces to

$$
\frac{\partial \mathrm{A}_{3}}{\partial z}=\frac{-\pi \omega_{3}^{2}}{2 i k_{3} c^{2}} \mathrm{~N} \chi^{(3)}\left(-3 \omega_{1}, \omega_{1}, \omega_{1}, \omega_{1}\right) \mathrm{A}_{1}^{3} e^{-i \Delta k z}
$$


which may be solved yielding,

$$
\mathrm{A}_{3}(z)=\frac{i \pi \omega_{3}^{2}}{2 k_{3} c^{2}} \mathrm{~N} \chi^{(3)} \mathrm{A}_{1}^{3}\left(\frac{1-e^{-i \Delta k z}}{i \Delta k}\right)
$$

where $\Delta k=k_{3}-3 k_{1}$. Thus the intensity of the third harmonic wave is given by

$$
\mathrm{I}_{3}=\frac{c}{8 \pi} \mathrm{A}^{2}=\left(\frac{\pi^{2} \omega_{3}^{2} z^{2}}{c^{3} k_{3}}\right) N^{2}\left|\chi^{(3)}\right|^{2} \mathrm{I}_{1}^{3}\left[\frac{\sin (\Delta k z / 2)}{(\Delta k z / 2)}\right]^{2},
$$

which may be generalized to

$$
\mathrm{I}_{3} \propto \mathrm{N}^{2}\left|\chi^{(3)}\right|^{2} \mathrm{I}_{1}^{3}[\operatorname{sinc}(\Delta k z / 2)]^{2} z^{2}
$$

The $\operatorname{sinc}^{2}$ term represents the influence of phase matching on the process and will have its maximum value when the phases of the incident and harmonic beams are exactly equal, $\Delta k=0$.

\subsubsection{Phase Matching}

In general, the phase matching term will depend on the characteristics of the incident electric field. For a focused beam incident upon a gaseous medium with a gaussian profile

$$
E_{1}=\left(\frac{E_{o 1}}{1+i \xi}\right) e^{i k_{1} z} e^{-k_{1} r^{2} / b(1+i \xi)}
$$

where $b$ is the laser confocal parameter and $\xi=2\left(z-z_{0}\right) / b$ is the position of the focus The amplitude of the $q$ th harmonic wave is

$$
\mathrm{E}_{o q}=\frac{i q \pi^{2} b}{2 n_{q} \lambda_{1}} \chi^{(q)} \frac{\mathrm{E}_{o 1}^{q}}{(1+i \xi)} e^{-q k_{1} r^{2} / b(1+i \xi) \Delta k_{q} z_{0}} \int_{-\xi^{\prime}}^{\xi} \frac{e^{i b \Delta k_{q} \xi^{\prime \prime} / 2}}{\left(1+i \xi^{\prime \prime}\right)^{q-1}} d \xi^{\prime \prime}
$$

where $\xi^{\prime}=2 z_{o} / b$ and

$$
\int_{-\xi^{\prime}}^{\xi} \frac{e^{i b \Delta k \xi^{\prime \prime} / 2}}{\left(1+i \xi^{\prime \prime}\right)^{q-1}} d \xi^{\prime \prime}=F(b \Delta k)
$$

is called the phase matching integral. Thus, the harmonic is also a Gaussian with the same confocal parameter and beam waist location as the incident radiation and with 
a far field divergence angle which is $1 / q^{1 / 2}$ that of the fundamental. In general, the intensity of the $q$ th harmonic will then be given by

$$
\mathrm{I}_{q} \propto \mathrm{N}^{2}\left|\chi^{(q)}\right|^{2} \mathrm{I}_{\mathfrak{1}}^{q}[F(b \Delta k)]^{2} z^{2} .
$$

$F(b \Delta k)$ may be examined for several limits of the value of $b$. When $b$ is much shorter than the length of the medium, the "tight focusing" limit, the limits of integration in 1.66 may be taken to $\pm \infty$ yielding

$$
F(b \Delta k)= \begin{cases}0 & \Delta k>0 \\ \frac{2 \pi(-b \Delta k / 2) q-2 e^{\Delta \Delta k / 2}}{(q-2) !} & \Delta k<0\end{cases}
$$

which has its maximum for $\Delta k=-2(q-2) / b<0$. If the medium is much shorter than $b$, then $\xi^{\prime \prime}<<1$ and 1.66 becomes

$$
|F(b \Delta k)|^{2}=\left(\frac{2 L}{b}\right)^{2} \operatorname{sinc}^{2}[(\Delta k-2(q-1) / b) L / 2]
$$

which has its maximum value at $\Delta k=-2(q-1) / b$. In both cases, best phase matching occurs for negative dispersion, $\Delta k<0$.

\subsection{6 "Weak" versus "Strong" Fields}

Clearly the approximation of the laser field as a small perturbation on the atomic field must become invalid as the intensity is increased. The field at which this occurs can be estimated by examining the multiphoton matrix element which determines the probability for the absorption of $N$ photons. When the probability of the $N+1$ photon process is the same magnitude as the probability of the $N$ photon process, lowest order perturbation theory is no longer valid.

$$
M_{f i}^{(N+1)}(\omega)=\sum_{j} \sum_{k} \cdots \sum_{n+1} \frac{<f|e \mathbf{E} \cdot \mathbf{r}| j><j|e \mathbf{E} \cdot \mathbf{r}| k>\cdots<n+1|e \mathbf{E} \cdot \mathbf{r}| i>}{\left(\omega_{j i}-(N) \omega\right)\left(\omega_{k i}-(N-1) \omega\right) \cdots\left(\omega_{n+1}-\omega\right)}
$$

is the $N+1$ photon matrix element and the $N$ photon matrix element is

$$
M_{f i}^{(N)}(\omega)=\sum_{j} \sum_{k} \cdots \sum_{n} \frac{<f|e \mathbf{E} \cdot \mathbf{r}| j><j|e \mathbf{E} \cdot \mathbf{r}| k>\cdots<n|e \mathbf{E} \cdot \mathbf{r}| i>}{\left(\omega_{j i}-(N-1) \omega\right)\left(\omega_{k i}-(N-2) \omega\right) \cdots\left(\omega_{n i}-\omega\right)} .
$$


The field at which these are of the same magnitude can be calculated by setting their ratio equal to 1 .

$$
\frac{\left|M_{f i}^{(N+1)}(\omega)\right|}{\left|M_{f i}^{(N)}(\omega)\right|} \sim \frac{|<n+1| e \mathrm{E} \cdot \mathbf{r}|i>|}{\left|\left(\omega_{j i}-N \omega\right)\right|}=\frac{|e E|\left|r_{n+1, i}\right|}{\left|\left(\omega_{j i}-N \omega\right)\right|}
$$

or

$$
|E| \sim \frac{\left(\omega_{j i}-N(\nu)\right.}{e\left|r_{n+1, i}\right|} .
$$

Now if $r_{n+1, i} \sim a_{o}$, where $a_{o}=5.29 \times 10^{-9} \mathrm{~cm}$ is the Bohr radius, a simple example can be solved. For the case of hydrogen the 1s-2p transition has an energy of 10.15 $\mathrm{eV}$. At $580 \mathrm{~nm}$ the photon energy is $2.14 \mathrm{eV}$ and four photons lie just below the $2 \mathrm{p}$ level yielding,

$$
|\mathrm{E}| \sim \frac{(10.15 \mathrm{eV}-8.56 \mathrm{eV})}{e a_{0}}=\frac{1.6 \mathrm{~V}}{a_{0}}=3 \times 10^{8} \mathrm{~V} / \mathrm{cm}
$$

or an intensity of $\mathrm{I}=1.2 \times 10^{14} \mathrm{~W} / \mathrm{cm}^{2}$. This intensity is readily achievable with currently available laser systems so while perturbation theory provides a good starting point for understanding harmonic generation, other approaches will be required to examine the problem in the strong field regime.

\subsection{7 "Strong" Field Regime}

In order to produce very high order harmonics, laser intensities $\sim 10^{13}-10^{14} \mathrm{~W} / \mathrm{cm}^{2}$ are required. The laser electric field strength at such an intensity is approaching that of the atomic field and is clearly no longer a small perturbation. This is the regime of so-called nonperturbative processes. Time-dependent approaches to this problem have included solution of the Schrödinger equation [kulan89, kraus92b] and Floquet calculations.[potv189] One-dimensional approximations [eberl89, eberl89b], classical methods [banda90, chu90] and other models [shore87, becke90, biede89, sunda90] have also provided insight into the problem. Despite the single-atom nature of these calculations, they in general agree qualitatively with experimental data. Other work [lhuil91] has extended these calculations to include the influence of phase-matching. 
Theoretically,[kraus92] it has been seen that the energy at which the cutoff occurs in the single-atom response is well-approximated by

$$
\mathrm{E}_{\text {cutoff }}=\mathrm{I}_{\mathrm{P}}+3 \mathrm{U}_{\mathrm{P}}
$$

where $I_{P}$ is the ionization potential of the atom and

$$
\mathrm{U}_{\mathrm{P}}(\mathrm{eV})=\frac{e^{2} \mathrm{E}^{2}}{4 m \omega^{2}}=9.33 \times 10^{-14} \mathrm{I}(\mathrm{W} / \mathrm{cm})^{2} \lambda^{2}(\mu \mathrm{m})
$$

is the ponderomotive or "quiver" energy of the electron in the laser field. When the contribution of phase matching is added to the single-atom response the factor of 3 may be diminished to approximately 2 in equation 1.75.[1huil93b]

More recently, in an effort to provide physical insight into questions such as the location of the cutoff a two-step semiclassical model [kulan93, schaf93, corku93] has been suggested. In the semi-classical picture, the atomic potential is lowered by the presence of the intense laser field allowing the electron to tunnel through the potential barrier. The subsequent motion of the electron in the field is treated as that of a free electron and the classical trajectories of the electron are examined. If the trajectory is one which returns the electron to the nucleus, the electron may recombine to the ground state and emit a harmonic photon. However, if the trajectory . is such that the electron does not return to the nucleus, the atom will simply ionize and no harmonic production will be observed. Classical simulations show that the maximum energy the electron may have upon returning to the nucleus is $3 \mathrm{U}_{\mathrm{P}}$, in good agreement with quantum mechanical calculations. A simple, analytic and fully quantum-mechanical theory valid in the tunneling limit has also been used to examine this problem.[lewen94] This theory is valid in a low frequency high intensity limit, where $U_{P} \geq I_{P}$, and for high order harmonics, those with energies greater than the ionization potential, and includes many quantum mechanical effects which are not included in the classical approach. 


\subsection{Research Program}

Two major goals drove the research presented herein. The first was to further the understanding of the basic physics issues underlying harmonic generation. A series of preliminary experiments were done to confirm the predictions of lowest order perturbation theory and to provide a general survey of harmonic generation in the rare gases. An experiment was then devised to test the range of validity of the two-step semiclassical model of harmonic generation. The quantum model of Lewenstein et al.[lewen94] was used to model the data.

The development of high order harmonic generation as a source of coherent, tunable, XUV radiation was the second goal. This required characterization of the harmonic "source" including an examination of the spatial coherence properties of the radiation produced. Finally, the harmonic source was utilized to perform measurements of the photoionization cross section of several target species.

\subsection{Plan of the Manuscript}

This manuscript is organized according to the motivation presented above. Chapter 2 presents a detailed description of the experimental facility. Both of the laser systems utilized as well as the experimental set-up required for harmonic generation are discussed and a detailed analysis of the short pulse dye laser system is included. This is followed by Chapter 3 which details the results of preliminary experiments to confirm the predictions of perturbation theory and spectra are presented for each of the rare gases studied. Chapter 4 begins with a detailed presentation of the twostep semiclassical model of harmonic generation and a description of the experiment on the influence of ellipticity devised as a test of its predictions. The experimental results are presented next and are subsequently modeled using the quantum model developed by Lewenstein, et al.,[lewen94].

The focus then shifts to the development of the harmonic "source" in Chapter 5. The first section details the characterization of the source and measurements of the 
spatial profiles of the harmonics. The concluding section of this chapter presents the results of photoionization experiments conducted utilizing harmonic radiation as the photon source. The manuscript will conclude with Chapter 6 which will summarize the work presented and suggest future directions in which to extend this work. 


\section{Chapter 2}

\section{Experimental Facility}

\subsection{Short Pulse Dye Laser}

In order to provide a tunable source of harmonic radiation, a high power dye laser system was utilized. The system described here is capable of providing focused irradiance in excess of $10^{14} \mathrm{~W} / \mathrm{cm}^{2}$ tunable from 570 to $640 \mathrm{~nm}$ with an amplified spontaneous emission (ASE) background of less than $10^{-4}$.

The laser system,[perry89] shown in Figure 2.1, begins with a stabilized, modelocked Nd:YAG (neodymium ${ }^{3+}$ : yttrium aluminum garnet) oscillator (Quantronix model $4126 \mathrm{MLSH}$ ) which produces a $76 \mathrm{MHz}$ train of mode-locked $100 \mathrm{psec}$ pulses at $1.064 \mu \mathrm{m}$ with an average power of $16 \mathrm{~W}$. The leakage through the cavity end mirror is monitored continuously by a fast photodiode to allow for easy adjustment of the cavity length. A typical profile is shown in Figure 2.2. The $1.064 \mu \mathrm{m}$ beam is focused into a $5 \mathrm{~mm}$ potassium titanyl phosphate (KTP) crystal to an $80 \mu \mathrm{m}$ spot diameter yielding a peak power density of $42 \mathrm{MW} / \mathrm{cm}^{2}$ and frequency converted via type II second-harmonic generation. The $532 \mathrm{~nm}$ pulse train is separated from the fundamental by a dichroic beam splitter and $\sim 1.1 \mathrm{~W}$ is used to synchronously pump a linear cavity dye laser (Spectra Physics Model 3500) with rhodamine 6G in ethylene glycol as the gain medium. Figure 2.3 shows the output power of the dye oscillator as a function of wavelength for these conditions. 


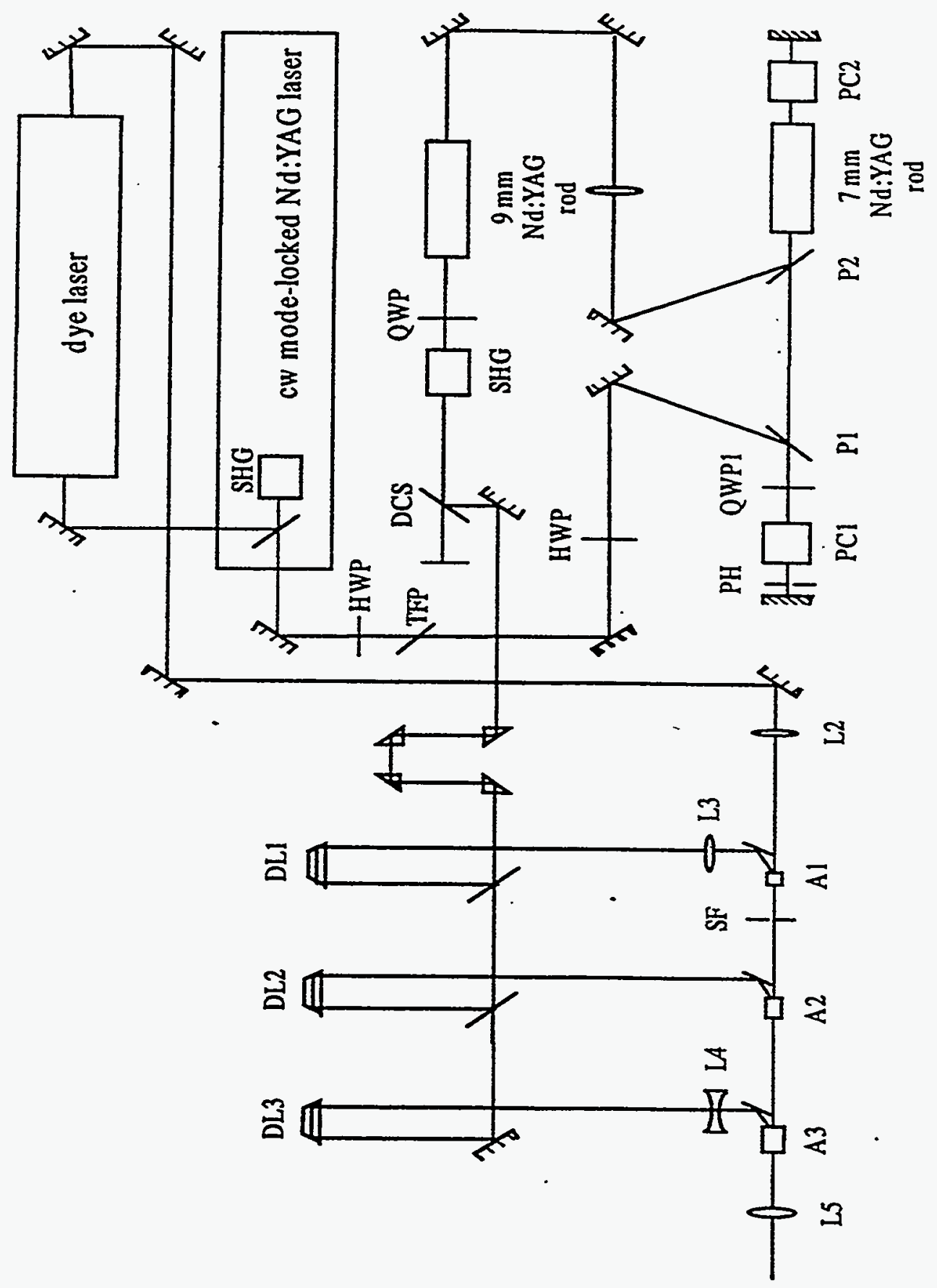

Figure 2.1: Schematic of the short pulse high power dye laser system. (HWP = halfwave plate, QWP = quarter-wave plate, $\mathrm{SHG}=$ second harmonic generation, $\mathrm{PC}=$ Pockels cell, TFP, $P=$ thin film polarizer, $\mathrm{L}=$ lens, $\mathrm{SF}=$ spatial filter, $\mathrm{DL}=$ delay line, $\mathrm{DCS}=$ dichroic beam splitter, $\mathrm{PH}=$ pinhole, $\mathrm{A}=$ dye amplifier cuvette) 


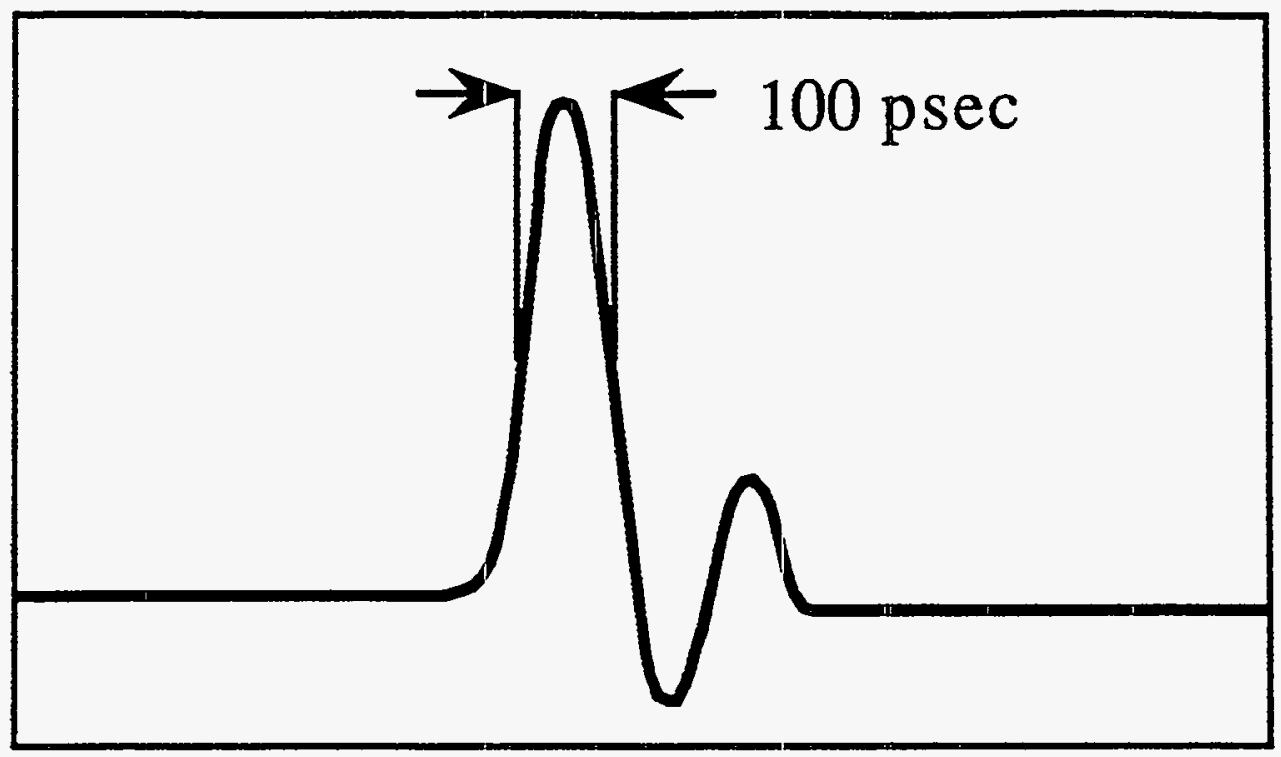

Figure 2.2: The temporal pulse profile of the mode-locked Nd:YAG oscillator. The secondary pulse is due to ringing in the fast photodiode.

By exactly matching the optical cavity lengths of the pump laser and the dye oscillator, the population inversion in the gain medium is generated and restored at a time which is synchronized to the cavity round-trip time. During each cavity round trip the circulating dye pulse makes two passes through the gain medium, each time rapidly depleting the the excited state population of the laser dye. When the cavity lengths are exactly matched, pulses of 2 psec duration are produced. By shortening the oscillator cavity length relative to that of the pump laser, the pulsewidth may be decreased to $800 \mathrm{fsec}$. The presence of additional pulses circulating in the cavity is observed for very short cavity lengths as lobes on the autocorrelation of the.amplified dye pulse. Very short cavity lengths correspond to the circulating dye pulse arriving as the leading edge of the pump pulse strikes the gain medium. After the dye pulse has passed, the remaining pump pulse may bring the laser above threshold again, allowing a second pulse to begin circulating in the cavity. Shown in Figure 2.4 are a series of autocorrelations depicting the evolution of the pulsewidth produced as a function of cavity length. These were obtained by the technique of noncollinear, background free autocorrelation by type I second harmonic generation in a potassium dihydrogen 


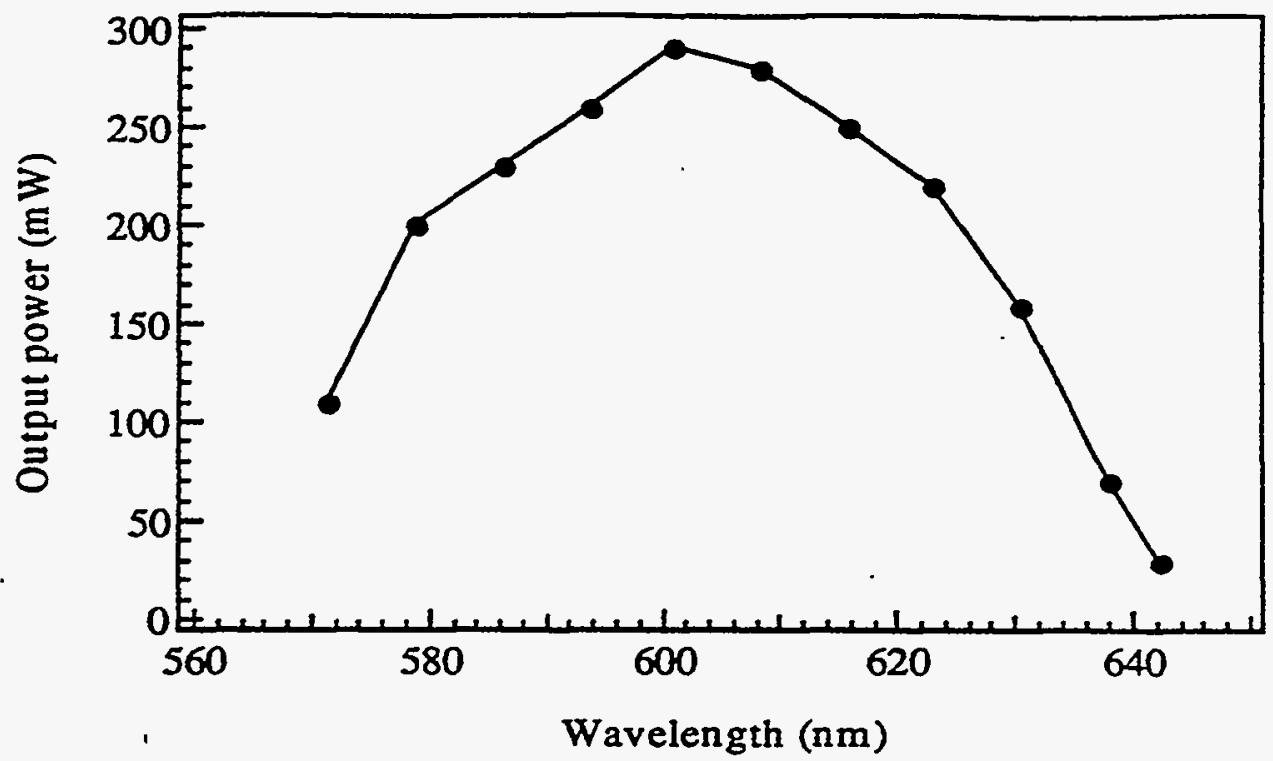

Figure 2.3: Output power of the dye oscillator as a function of wavelength with rhodamine $6 \mathrm{G}$ in ethylene glycol as the gain medium. The oscillator is continuously tunable from $575-640 \mathrm{~nm}$. Extending this range requires changing the cavity optics as well as the dye utilized.

phosphate (KDP) crystal using a Spectra Physics Model 409 autocorrelator. The lobes surrounding the main pulse are due to a secondary pulse produced after the main pulse circulating in the cavity. Because the second order autocorrelation function is symmetric, the lobe appears on both sides of the autocorrelation.

The frequency is tuned by adjusting the angle of a two-plate birefringent filter placed at Brewster's angle within the cavity. Rotating the filter about an axis normal to its surface causes the polar angle between the optic and laser axes to change, thus altering the wavelength whose retardation is an integral number of wavelengths. The elliptically polarized frequencies will experience additional cavity losses at other Brewster's angle optics causing them to fall below the lasing threshold of the cavity. However, incomplete polarization by a single plate filter can result in sidebands or additional frequencies circulating in the cavity. The active bandwidth is further reduced and the sidebands suppressed by the introduction of a second birefringent plate of greater thickness. 


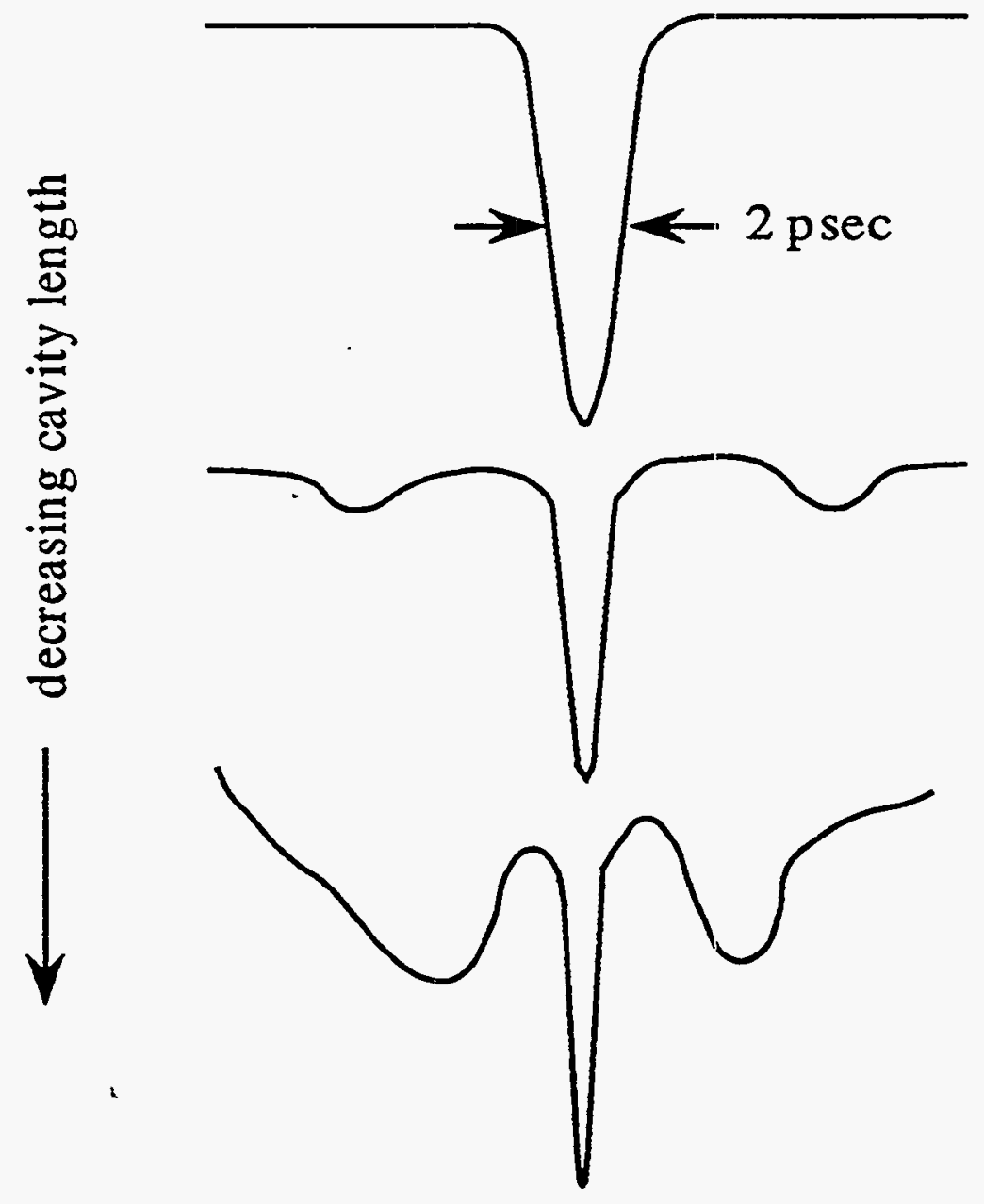

Figure 2.4: Autocorrelation of dye oscillator pulses as a function of cavity length. 
A single pulse from the dye laser train will be amplified in a three state dye amplifier pumped by $532 \mathrm{~nm}$ radiation. One of the primary problems in such amplification schemes traditionally where the amplifiers are pumped by $532 \mathrm{~nm}$ pulses of nanosecond duration is the high level of ASE present in the output, often several percent of the main pulse. By pumping the amplifiers with shorter pulses ( $100 \mathrm{psec}$ ), the pulse being amplified is able to extract the gain from the amplifier before there is time for a significant buildup of ASE and the contrast ratio between the amplified pulse and the ASE background improves from one to several orders of magnitude. By using a regenerative amplifier to produce the pump radiation, the laser system may be kept compact and both cost and complexity are reduced. [sizer81]

In a regenerative amplifier, a single light pulse is trapped in a laser resonator and allowed to make many passes through the gain medium. When the pulse has reached its maximum amplitude it is switched out of the cavity. The output pulse has the temporal characteristics of the seed pulse while the spatial mode is determined by the resonator design: A large mode volume is desirable in order to maximize energy extraction but good beam quality must be maintained as well. While unstable resonators generally support large mode volumes, the beam quality is poor due to diffractive output coupling. A stable TEM ${ }_{00}$ resonator provides excellent beam quality but is very sensitive to alignment when the circulating mode is large.[perry89] For the best combination of large mode volume, good beam quality and ease of alignment, a diffraction filtered resonator [pax88] was chosen for this application.

The residual $1.064 \mu \mathrm{m}$ light from the Nd:YAG oscillator passes through the dichroic splitter and is sent through an attenuator consisting of a half-wave plate followed by a thin film polarizer which allows the amount of $1.064 \mu \mathrm{m}$ power to be continuously varied. The s-polarized component of the pulse train reflects off polarizer P1 through quarter-wave plate QWP2 and Pockels cell PC1. The pulse diffracts from pinhole PH1 and makes its second pass through PC1 and QWP2. The now p-polarized pulse is then transmitted through $\mathrm{P} 1 . \mathrm{PC} 1$ is fired at its quarter-wave voltage $(3500 \mathrm{~V})$ immediately after the trapped pulse has made its second pass, thus 
causing the rest of the pulse train to experience a full-wave retardation and be reflected by P1. After 9 round trips, the pulse is switched out of the cavity by raising Pockels cell PC2 to its quarter-wave voltage and returning it to s-polarization which will be rejected by polarizer $\mathrm{P} 2$.

The cavity is designed such that when the beam makes its second pass through the $7 \mathrm{~mm} \times 100 \mathrm{~mm}$ Nd:YAG rod, the first minimum of the Airy pattern produced by the diffraction from the pinhole is clipped by the edges of the rod. The measured single pass gain of this head as a function of pump energy is shown in Figure 2.5. This

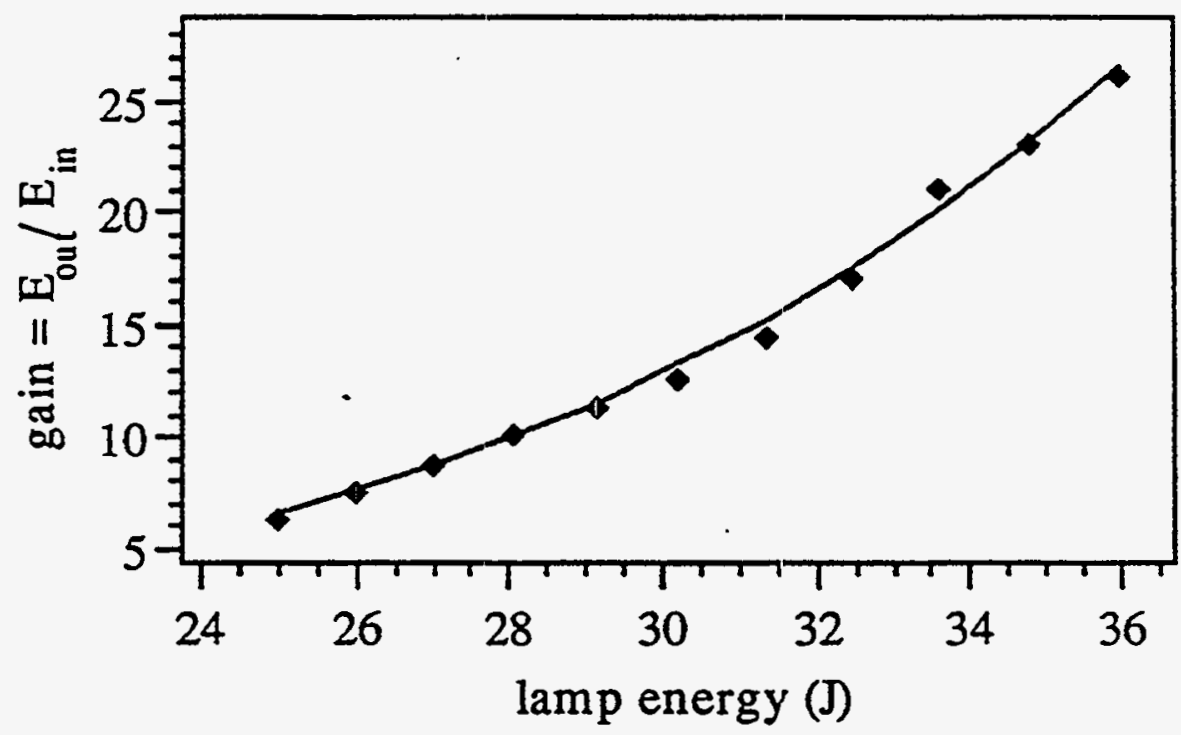

Figure 2.5: The measured single pass gain $G=E_{\text {out }} / E_{\text {in }}$ as a function of the pump energy in joules. The solid line is an exponential fit to the data.

allows for maximum filling of the rod without introducing severe beam distortion. Once the length of the cavity and placement of the laser head are determined, the number of round trips required to achieve the desired gain level must be calculated. This is accomplished by examining the propagation of a light pulse through a gain medium.

The properties of the regenerative amplifier may be studied by solving the coupled rate equations for the photon density in the resonator and the population inversion in the medium. The energy level structure of $\mathrm{Nd}^{3+}$ is shown in Figure 2.6. Emission at 


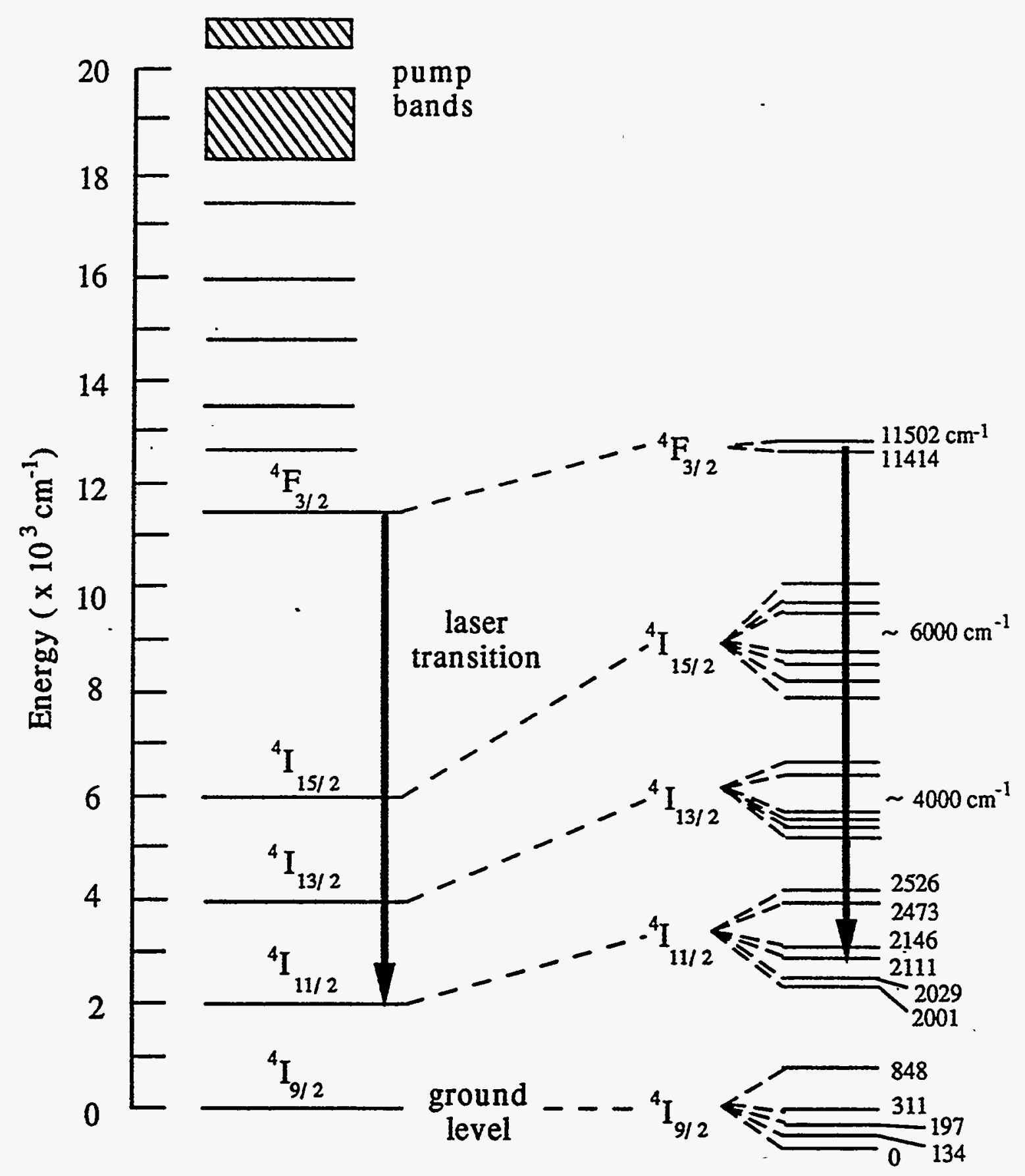

Figure 2.6: The energy level structure of $\mathrm{Nd}^{3+}$ with the $1.06 \mu$ m lasing transition indicated. 
$1.064 \mu \mathrm{m}$ results from the ${ }^{4} \mathrm{~F}_{3 / 2}$ to ${ }^{4} \mathrm{I}_{11 / 2}$ transition. $\mathrm{Nd}^{3+}$ is a four-level laser system where the relaxation of the pump bands into the upper laser level occurs very quickly so any population in the pump band may be neglected. If the populations of the upper and lower laser levels are denoted by $n_{2}$ and $n_{1}$ respectively and the photon density is denoted by $\phi(x, t)$, the photon transport equation is [frant63]

$$
\frac{\partial \phi}{\partial \mathrm{t}}+c \frac{\partial \phi}{\partial x}=\sigma c \phi\left(n_{2}-n_{1}\right)
$$

where $\sigma$ is the emission cross section and $c$ is the velocity of light in the medium. The right-hand side of equation 2.1 is simply the difference between the rate of stimulated emission and the rate of absorption where spontaneous emission has been neglected. The rate of change of the populations of the upper and lower levels must also be related to this difference, yielding the population rate equations

$$
\begin{gathered}
\frac{\partial n_{1}}{\partial \mathrm{t}}=\sigma c \phi\left(n_{2}-n_{1}\right) \\
\frac{\partial n_{2}}{\partial \mathrm{t}}=-\sigma c \phi\left(n_{2}-n_{1}\right) .
\end{gathered}
$$

These equations may be expressed in terms of the population inversion, $\Delta(x)=$ $n_{2}-n_{1}$, giving

$$
\frac{\partial \Delta}{\partial \mathrm{t}}=-2 \sigma c n \Delta
$$

and

$$
\frac{\partial \phi}{\partial \mathrm{t}}+c \frac{\partial \phi}{\partial x}=\sigma c n \Delta
$$

By applying the boundary conditions $\phi_{0}(t)=\phi_{0}(0, t)$ and $\Delta_{0}(x)=\Delta_{0}(x,-\infty)$ these equations may be solved for arbitrary $\phi_{0}(t)$ and $\Delta_{0}(x)$ yielding

$$
\phi(x, \mathrm{t})=\frac{\phi_{0}(\mathrm{t}-x / c)}{1-\left\{1-\exp \left[-\sigma \int_{0}^{x} \Delta_{0}\left(x^{\prime}\right) d x^{\prime}\right]\right\} \exp \left[-2 \sigma c \int_{-\infty}^{\mathrm{t}-x / c} \phi_{0}\left(\mathrm{t}^{\prime}\right) d \mathrm{t}^{\prime}\right]}
$$

and

$$
\Delta(x, \mathrm{t})=\frac{\Delta_{0}(x) \exp \left[-\dot{\sigma} \int_{0}^{x} \Delta_{0}\left(x^{\prime}\right) d x^{\prime}\right]}{\exp \left[2 \sigma c \int_{-\infty}^{t-x / c} \phi_{0}\left(\mathrm{t}^{\prime}\right) d \mathrm{t}^{\prime}\right]+\exp \left[-\sigma \int_{0}^{x} \Delta_{0}\left(x^{\prime}\right) d x^{\prime}\right]-1}
$$


If the initial population inversion is assumed to be a constant, $\Delta_{0}(x)=\Delta_{0}$, these become

$$
\phi(x, t)=\frac{\phi(t-x / c)}{1+\left[e^{\left(-\sigma \Delta_{0} x\right)}-1\right] e^{-2 \sigma c \int_{-\infty}^{s-x / c} \phi_{0}\left(t^{\prime}\right) d t^{\prime}}}
$$

and

$$
\Delta(x, \mathrm{t})=\frac{\Delta_{0} e^{-\sigma \Delta_{0} x}}{\exp \left[2 \sigma c \int_{-\infty}^{t-x / c} \phi_{0}\left(\mathrm{t}^{\prime}\right) d t^{\prime}\right]+\left[e^{-\sigma \Delta_{0} x}-1\right]} .
$$

To model the behavior of the diffraction filtered resonator, the input pulse will be assumed to have a gaussian temporal profile,

$$
\phi_{0}(\mathrm{t})=\phi_{o} e^{-2 \ln 2 t^{2} / \tau^{2}},
$$

where $\tau$ is the full width at half maximum (FWHM) of the profile. As the pulse amplitude grows in the cavity, the population will be depleted eventually resulting in a decrease of the pulse amplitude. Cavity losses are $~ 95 \%$ due to the low throughput of the diffracting pinhole. For a typical pump energy of $36 \mathrm{~J}$, the small-signal gain $G=25.8$ yielding an inversion density of

$$
\begin{aligned}
\Delta_{0} \cdot & =\frac{\ln (G)}{\ell \sigma} \\
& =\frac{3.25}{10 \mathrm{~cm} \times 6.5 \times 10^{-19} \mathrm{~cm}^{2}} \\
& =5 \times 10^{17} \text { atoms } / \mathrm{cm}^{3} .
\end{aligned}
$$

For $1 \%$ doping, the density of $\mathrm{Nd}$ atoms is $1.38 \times 10^{20}$ atoms $/ \mathrm{cm}^{3}$ yielding an inversion fraction of $\sim 3 \times 10^{-3}$. The FWHM of the input pulse is $100 \mathrm{psec}$ and the length of the cavity is $165 \mathrm{~cm}$.

Figure 2.7 is a plot of the amplitude of the pulse after each round trip as a function of the number of round trips. The parameters for this calculation were $\tau=$ $100 \mathrm{psec}$, an effective beam cross sectional diameter of $2.75 \mathrm{~mm}$, input energy $\mathrm{E}_{\mathrm{in}}=$ $1.5 \mathrm{~nJ}$, cavity loss of $95 \%$ and an initial population inversion $\Delta_{o}=2.75 \times 10^{17} \mathrm{~cm}^{-3}$. Experimentally, the relative timing between Pockels cells 1 and 2 determines the number of round trips the pulse makes in the cavity. By setting the separation of the triggers to the Pockels cells to be $95 \mathrm{nsec}$ ( 9 round trips) apart, it is seen that 


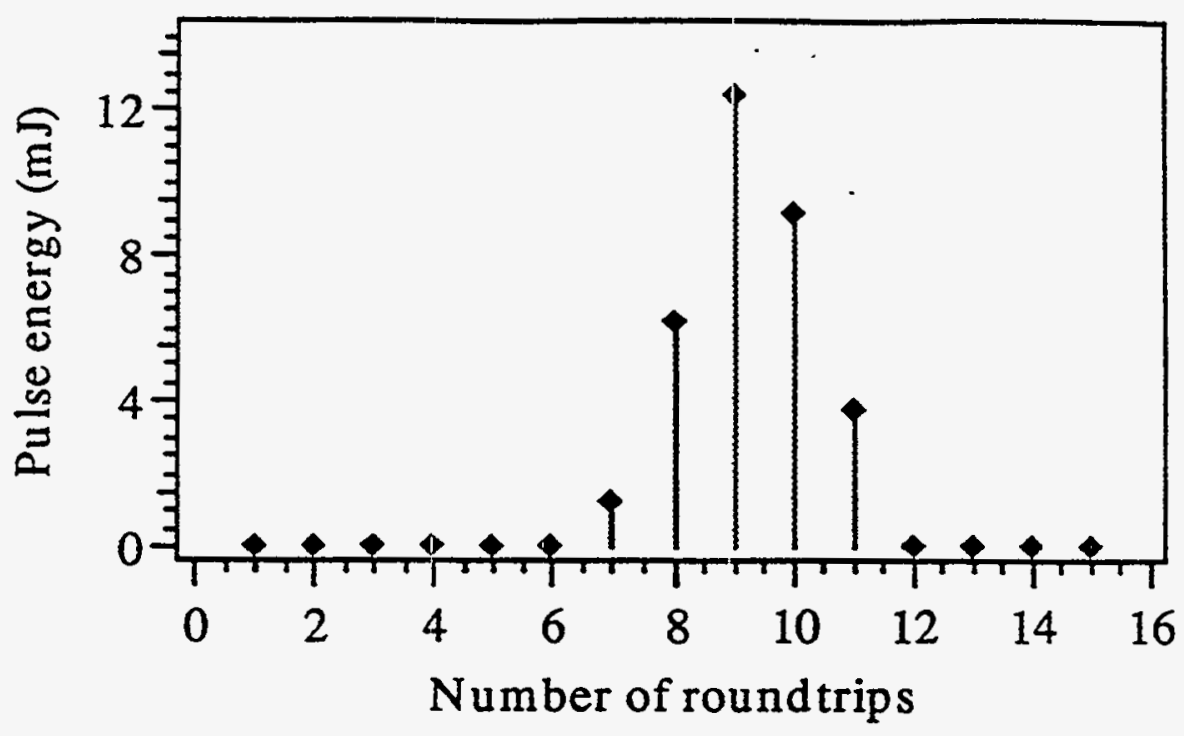

Figure 2.7: The pulse amplitude in the regenerative amplifier as a function of the number of cavity round trips as calculated for the case of a constant initial population inversion and a gaussian input pulse. The pulse has reached its maximum amplitude after 9 round trips.

the pulse amplitude is indeed at its maximum and the correct pulse will be switched out. Figure 2.8 shows the actual buildup in the resonator measured by placing a photodiode behind polarizer $\mathrm{P} 1$. The pulse energy after the regenerative amplifier is typically $\sim 15 \mathrm{~mJ}$ representing a gain of $\sim 10^{6}$.

After the pulse exits the regenerative amplifier it is collimated at $6.2 \mathrm{~mm}$ and amplified to approximately $300 \mathrm{~mJ}$ by making a single pass through a $9 \mathrm{~mm} \times 115$ $\mathrm{mm}$ Nd:YAG amplifier rod operated at a typical pump energy of $63 \mathrm{~J}$. The singlepass gain of the amplifier head as a function of pump energy is shown in Figure 2.9. The $6.2 \mathrm{~mm}$ beam waist is small enough to avoid severe diffraction effects due to the edges of the $9 \mathrm{~mm}$ rod. Thermal focusing in this rod and the potential for damage to turning optics for this beam limit the energy extracted from this amplifier as well as the repetition rate at which it may be operated. The system has been operated at $10 \mathrm{~Hz}$ but is typically operated at $5 \mathrm{~Hz}$ or less to minimize these effects as well as to avoid overloading the heat exchanging capacity of the cooling group which supplies 


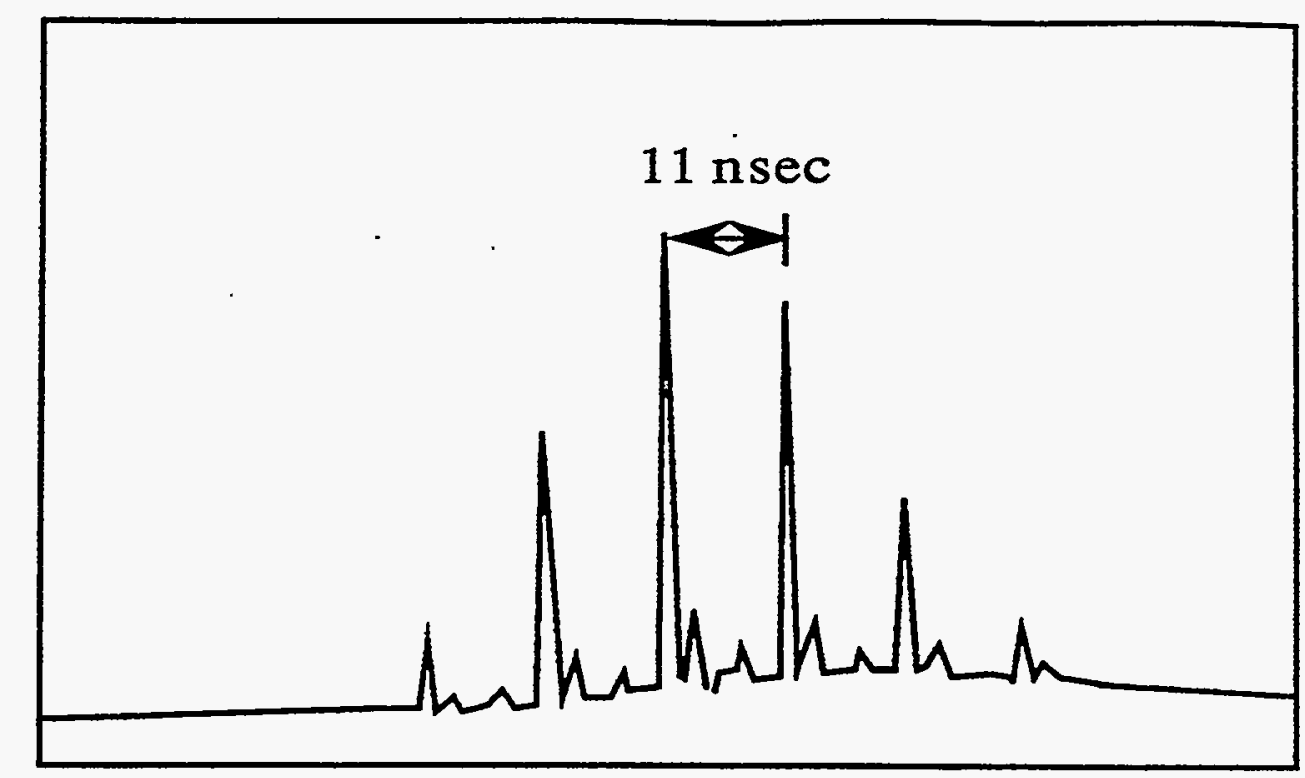

Figure 2.8: The experimentally observed pulse build up in the regenerative amplifier. The triggers to the Pockels cells are set $95 \mathrm{nsec}$ apart for maximum output, equivalent to 9 round trips. The pulse amplitude on the first several round trips is too small to be observed with the photodiode.

both the $7 \mathrm{~mm}$ head in the regenerative amplifier and the $9 \mathrm{~mm}$ amplifier head. The $1.064 \mu \mathrm{m}$ light is then passed through a half-wave plate and frequency converted to $532 \mathrm{~nm}$ in a $1 \mathrm{~cm}$ KDP crystal. The maximum energy at the second harmonic is $\sim 225 \mathrm{~mJ}$ representing a conversion efficiency of $75 \%$. Typically $65 \mathrm{~mJ}$ of $532 \mathrm{~nm}$ light is used to pump the dye amplifier chain and the pulse width after conversion is $\sim 75$ psec.

Initially the laser was internally triggered but the only early trigger available in this configuration is the "charge" command to the capacitor banks which has a very large $(\sim 200 \mu \mathrm{sec})$ jitter associated with it. For the experiments being considered here, the laser arrival must be precisely timed to coincide with the opening of a pulsed gas jet which releases a puff of gas only $250 \mu \mathrm{sec}$ long so a timing jitter as large as that associated with the "charge" command is clearly unacceptable. In order to circumvent this problem, the firing of the laser is controlled externally, fixing the "charge" command in time and allowing for very stable, early triggers. 


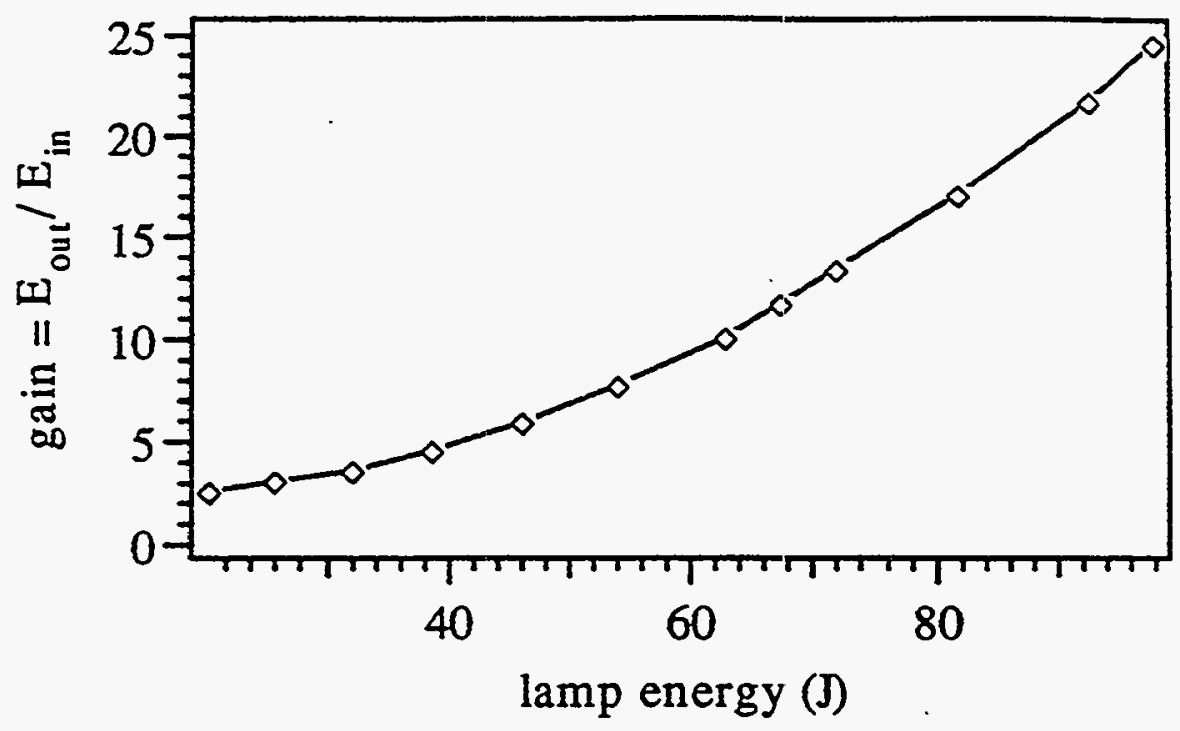

Figure 2.9: The measured single pass gain $G=E_{\text {out }} / E_{\text {in }}$ as a function of the pump energy in joules for the $9 \mathrm{~mm}$ amplifier head. The solid line is an exponential fit to the data.

In this configuration a pulse generator (Tektronix 2101) is used to generate the "charge command" which tells the control unit (Continuum CU551) to begin charging its capacitor banks. The repetition rate is set by varying the pulse period on the pulse generator. The power unit (Continuum PU420) then returns an "end-of-charge" signal to show that charging is complete. This signal triggers a Stanford Research Systems DG535 digital delay generator which then sends the command to discharge the capacitors across the flashlamps, the "fire" command. It also sends a trigger to the frequency scalar board (Continuum) which controls the timing of the Pockels cells. The rf output from the mode-locker of the Nd:YAG seed laser is also input to the scalar board. The board then counts a particular number of cycles of $\mathrm{rf}$ to begin its timing sequence to ensure that only one pulse from the $76 \mathrm{MHz}$ train is trapped in the regenerative amplifier. A schematic of the timing control system is shown in Figure 2.10.

Three longitudinally pumped flowing dye cells are used for amplification of the mode-locked pulses from the dye oscillator. The concentrations in the cells are $\sim$ 


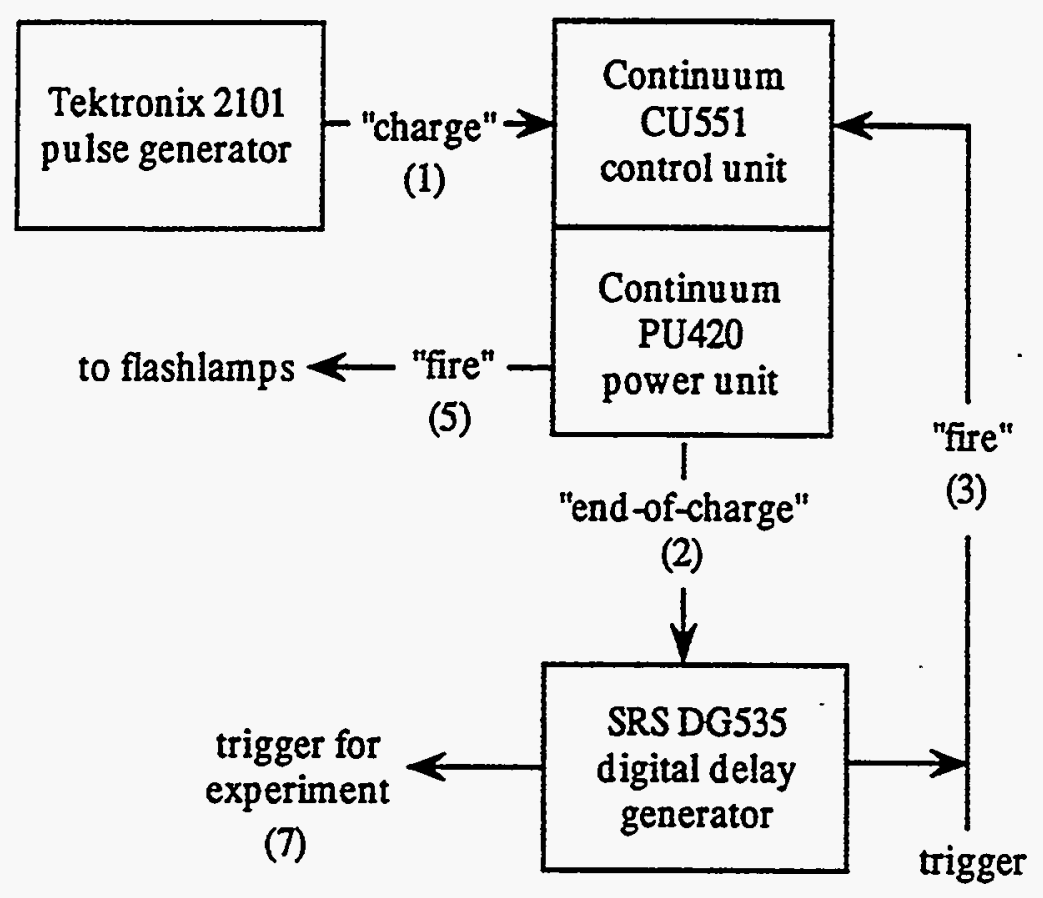

(3)

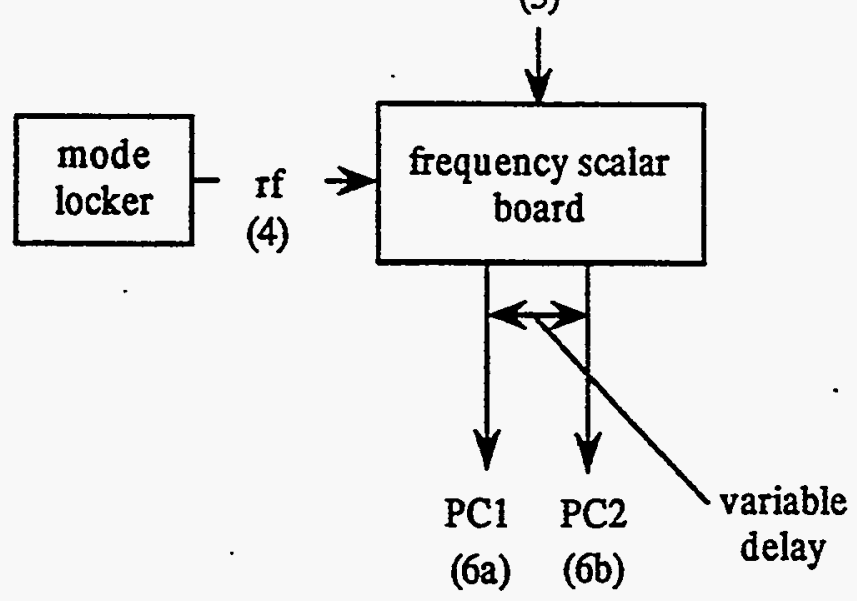

Figure 2.10: Schematic of the timing sequence for the regenerative amplifier. 
$2 \times 10^{-5}, 1 \times 10^{-5}$, and $0.8 \times 10^{-5} \mathrm{M}$ respectively. By utilizing several different dyes, the amplifier is operable over the range 570 to $640 \mathrm{~nm}$. The mode-locked dye pulse train is focused by lens $\ell 1$ into the first dye amplifier. A $50 \mu \mathrm{m}$ pinhole is placed at the focus of this lens, approximately $4 \mathrm{~cm}$ past the first amplifier cell. The beam is then allowed to diffract, with its central Airy maximum filling each of the two succeeding stages and is then recollimated by lens $\ell 2$. The $532 \mathrm{~nm}$ pump radiation is sent through an optical delay line in order to synchronize its arrival with that of one of the picosecond dye pulses. The beam is split into three components which are each sent through variable optical delay lines consisting of a roof prism on a translation stage. The amount of the pump beam diverted to each amplifier is $10 \%, 20 \%$, and $70 \%$ respectively. Precise timing of the pump pulse and the pulse to be amplified must be maintained in order to ensure maximum gain with minimum loss to ASE.[wokau82] The beam diameters in the amplifier cells are $\approx 0.6,7$ and $18 \mathrm{~mm}$ and the pump beam diameter is matched to this at each stage. The dye amplifier produces a maximum energy of $4 \mathrm{~mJ}$ with a pulse duration of less than one picosecond. The characteristics of the entire dye laser system are shown in Table 2.1. The dye amplifier chain is designed such that the incident pulse achieves the saturation fluence, $\mathrm{E}_{s}=h \nu / \sigma_{s}$ at approximately two-thirds the length of each dye cell. For fluences greater than this value, temporal distortion of the pulse is observed since the leading edge of the pulse will experience greater gain.

The technique of backgound-free single-shot autocorrelation is used to measure the duration of the amplified dye pulse on a shot-by-shot basis.[wyatt81] The beam entering the autocorrelator passes off of a plane diffraction grating and is divided by a beam splitter into two equal components. One of these beams traverses an arm containing an optical delay line so that the overlap between the two pulses may be controlled. The two pulses are focused into a thin KDP crystal and the second harmonic of their sum is generated along the axis dividing their paths. A diode array is used to detect this second harmonic signal. Figure 2.11 shows the autocorrelation of the amplified dye pulse with a calculated second order autocorrelation function 


\begin{tabular}{|l|l||}
\hline pulse width & variable between $.8 \mathrm{psec}$ and $4 \mathrm{psec}$ \\
\hline wavelength & $\begin{array}{l}\text { continuously tunable between } 570 \text { and } 640 \mathrm{~nm} \\
\text { by utilizing several amplifier dyes } \\
575-620 \mathrm{~nm} \text { for sulforhodamine } 101 \\
620-640 \mathrm{~nm} \text { for cresyl violet perchlorate }\end{array}$ \\
\hline pulse energy & $4 \mathrm{~mJ}$ maximum \\
\hline ASE & $\leq 10^{-4}$ of the amplified pulse energy \\
\hline beam quality & focusable to 1.5 times the diffraction limit \\
\hline
\end{tabular}

Table 2.1: Operating characteristics of the short pulse dye laser system. 
assuming a hyperbolic secant squared temporal distribution of width $\tau=1.24 \mathrm{psec}$, corresponding to a pulse width of $800 \mathrm{fsec}$ is shown as a solid line.

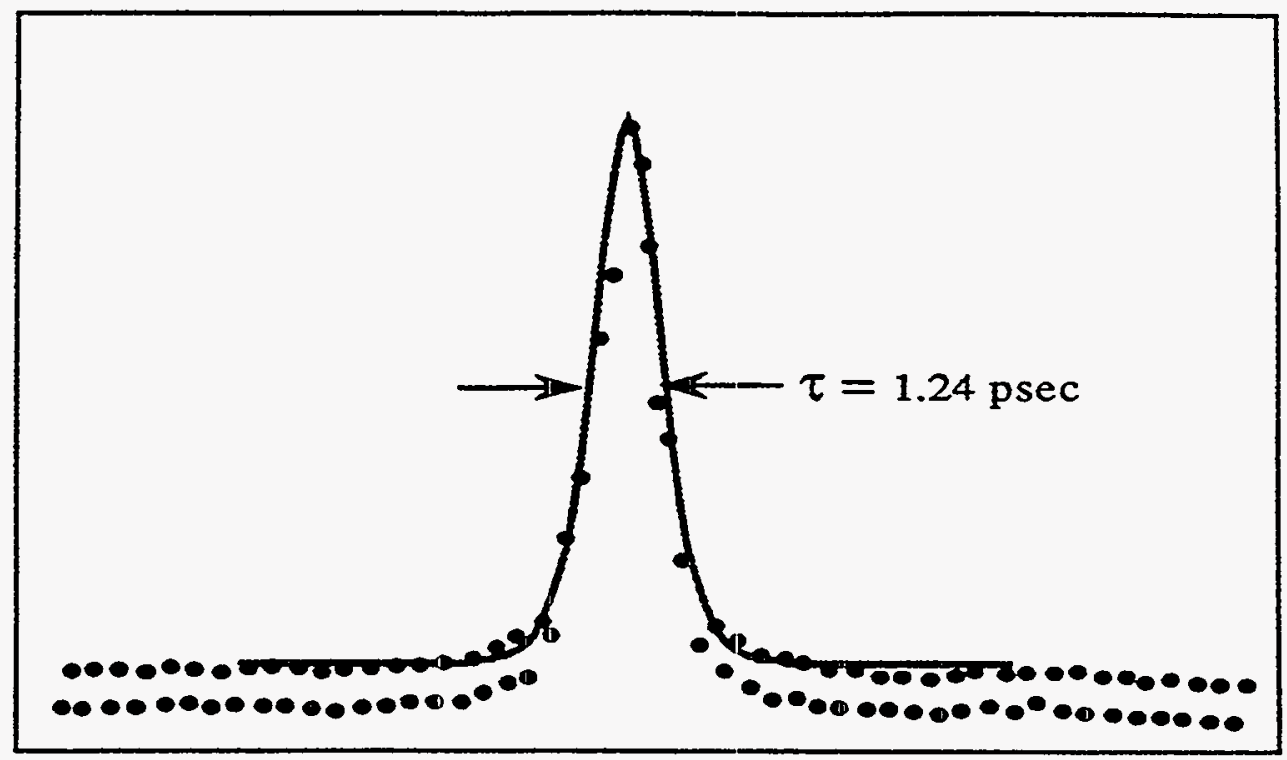

Figure 2.11: Single-shot, autocorrelation of the amplified dye pulse. The solid curve is a calculation of the second order autocorrelation function for a hyperbolic secant squared pulse of $\tau=1.24 \mathrm{psec}$ width. The actual pulse width is then $\Delta t=\tau / 1.55=$ 800 fsec.

For experiments, the laser may be operated as a full system producing picosecond tunable dye laser pulses or the regenerative amplifier-power amplifier pair may be used alone as a source of radiation at either $1.064 \mu \mathrm{m}$ or $532 \mathrm{~nm}$ with pulse durations of 100 and 70 psec respectively.

\subsection{Cr:LiSAF Laser System}

In order to scale these experiments to higher intensities a higher power laser system was required. Laser systerns based on chirped pulse amplification [stric85, maine88] can offer the combination of compactness, moderate energies and very short pulses which, when focused, can easily produce intensities $>10^{14} \mathrm{~W} / \mathrm{cm}^{2}$. Such a system, employing $\mathrm{Cr}: \mathrm{LiSrAlF}_{6}$ (LiSAF)[payne89] as the gain medium, was utilized for these 
experiments.[ditmi93, ditmi94a]

A schematic of this system is shown in Figure 2.12. The seed pulse is generated by a self mode-locked Ti:Sapphire oscillator (Coherent Mira 900 ) which produces a $76 \mathrm{MHz}$ train of transform limited $110 \mathrm{fsec}$ pulses of energy $8 \mathrm{~nJ}$. In order to prevent damage which would occur from the amplification of such a short pulse due to very high peak powers in the amplifiers and subsequent nonlinear effects (self-focusing, self-phase modulation), the pulse train is collimated and sent to a diffraction grating pulse stretcher where it is stretched temporally from $100 \mathrm{fsec}$ to $500 \mathrm{psec}$.[marti87] The stretcher consists of two 1800 line/mm gold-coated holographic diffraction gratings and two $60 \mathrm{~cm}$ focal length cemented achromatic doublet lenses which are corrected for chromatic and spherical aberration at $825 \mathrm{~nm}$. Due to losses in the stretcher, the pulse energy is reduced to $2 \mathrm{~nJ}$.

The pulse train is then injected into a ring-geometry regenerative amplifier operating at a $1-10 \mathrm{~Hz}$ repetition rate [perry92] where a single p-polarized pulse is selected for amplification by applying half wave voltage to a Pockels cell used in combination with a half wave plate to provide a full wave retardation to the trapped pulse. The $4 \mathrm{~m}$ long cavity is TEM To stable with the mode being defined by a $1.5 \mathrm{~m}$ focal length lens and a $2 \mathrm{~mm}$ aperture within the cavity. The pulse is amplified in a $4 \mathrm{~mm} \times$ $50 \mathrm{~mm}$ LiSAF rod and makes 37 round trips to achieve a total gain of $5 \times 10^{6}$. The pulse is ejected from the regenerative amplifier by returning the voltage on the Pockels cell to ground and is typically $12-15 \mathrm{~mJ}$ in energy. The gaussian output beam is then truncated by a $2 \mathrm{~mm}$ serrated aperture which serves to give the pulse a flat top spatial profile for greater fill factor in subsequent amplifiers. To ensure the propagation of the flat top throughout the rest of the system, the aperture is relay imaged. The pulse then makes a single pass through a $4 \mathrm{~mm}$ LiSAF amplifier to restore the energy lost at the serrated aperture.

Next the image is magnified in an air spatial filter by a factor of 2.9 resulting in a beam diameter of $6.1 \mathrm{~mm}$. The pulse is amplified by single-passing two $9.5 \mathrm{~mm} \times$ $115 \mathrm{~mm}$ LiSAF rods. The net gain here is 36 and energies greater than $550 \mathrm{~mJ}$ can 


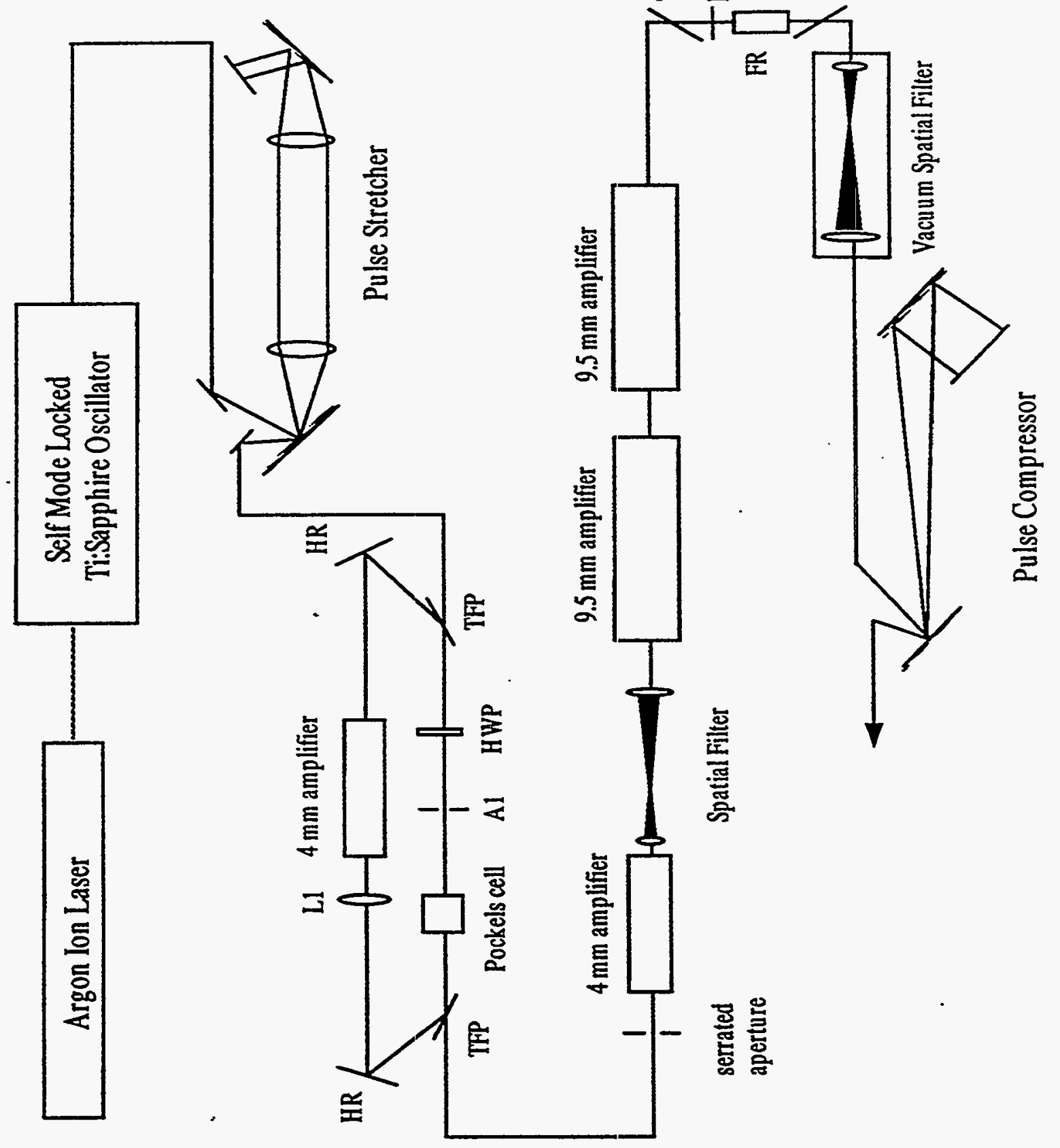

Figure 2.12: Schematic of the Cr:LiSAF laser system. (HR $=$ high reflector, $\mathrm{L}=$ lens, $\mathrm{TFP}=$ thin film polarizer, $\mathrm{A}=$ aperture, $\mathrm{HWF}=$ half-wave plate, $\mathrm{FR}=$ Faraday rotator) 
be achieved. The repetition rate of the system is limited by the rate at which these amplifiers can be fired and was .3-1 $\mathrm{Hz}$ depending upon the output energy desired. A Faraday rotator and half-wave plate are placed after these amplifiers to prevent back reflections which could damage the system. The pulse is then sent through a vacuum spatial filter to remove high order spatial frequencies and the spatial profile is magnified to $4.2 \mathrm{~cm}$ (magnification $=6.8$ ) to remain below the damage threshold of the gold-coated diffraction gratings in the compressor. The energy input to the compressor is limited to $510 \mathrm{~mJ}$ due to losses in the spatial filter as. well as those off of optics in the isolation stage.

The pulse is then double passed in a grating pair pulse compressor with overall efficiency of $55 \%$ resulting in output energies of up to $280 \mathrm{~mJ}$. An autocorrelation of the amplified pulse assuming a sech ${ }^{2}$ profile yields a pulse width of 135 fsec. For harmonics experiments the laser was typically operated in the range from a few $\mathrm{mJ}$ (regenerative amplifier only, $5 \mathrm{~Hz}$ repetition rate) to $50 \mathrm{~mJ}$ (both $9.5 \mathrm{~mm}$ amplifiers firing, $.3 \mathrm{~Hz}$ repetition rate).

\subsection{Experimental Apparatus}

These experiments on the production of harmonic radiation were accomplished by focusing a laser beam into a dense gaseous medium and detecting the spectrally dispersed radiation produced. The basic experimental set-up used is shown in Figure 2.13 .

The laser beam is sent to the experimental apparatus via an air path of several meters. An uncoated optical flat acts as a beam splitter, diverting four percent of the incident beam to a calibrated photodiode which will record the laser energy on each shot. The beam is then focused into a vacuum chamber by a spherical lens anti-reflection (AR) coated for the wavelength of interest ( $825 \mathrm{~nm}$ for the Cr:LiSAF laser system, broadband AR coating for $580-620 \mathrm{~nm}$ for the dye laser). The distance which the focused beam must propagate in air is minimized by placing an extension 
$5=0$
0
$\frac{1}{0} \equiv$

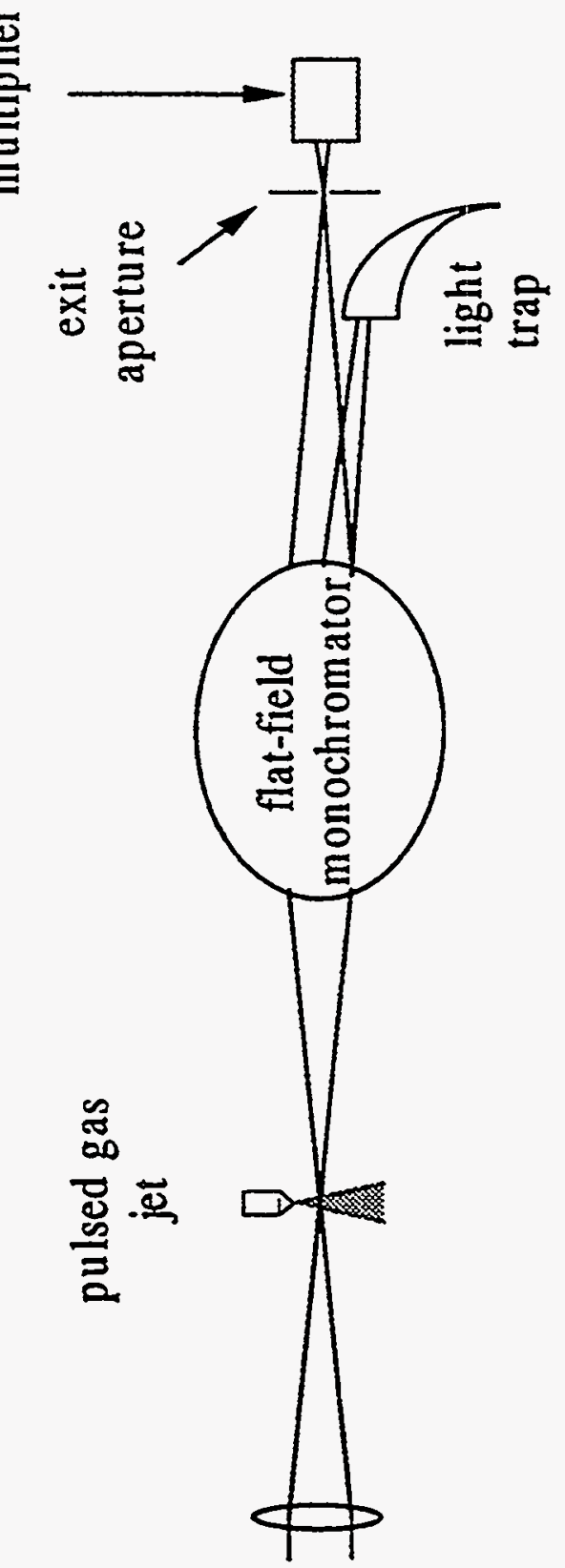

8
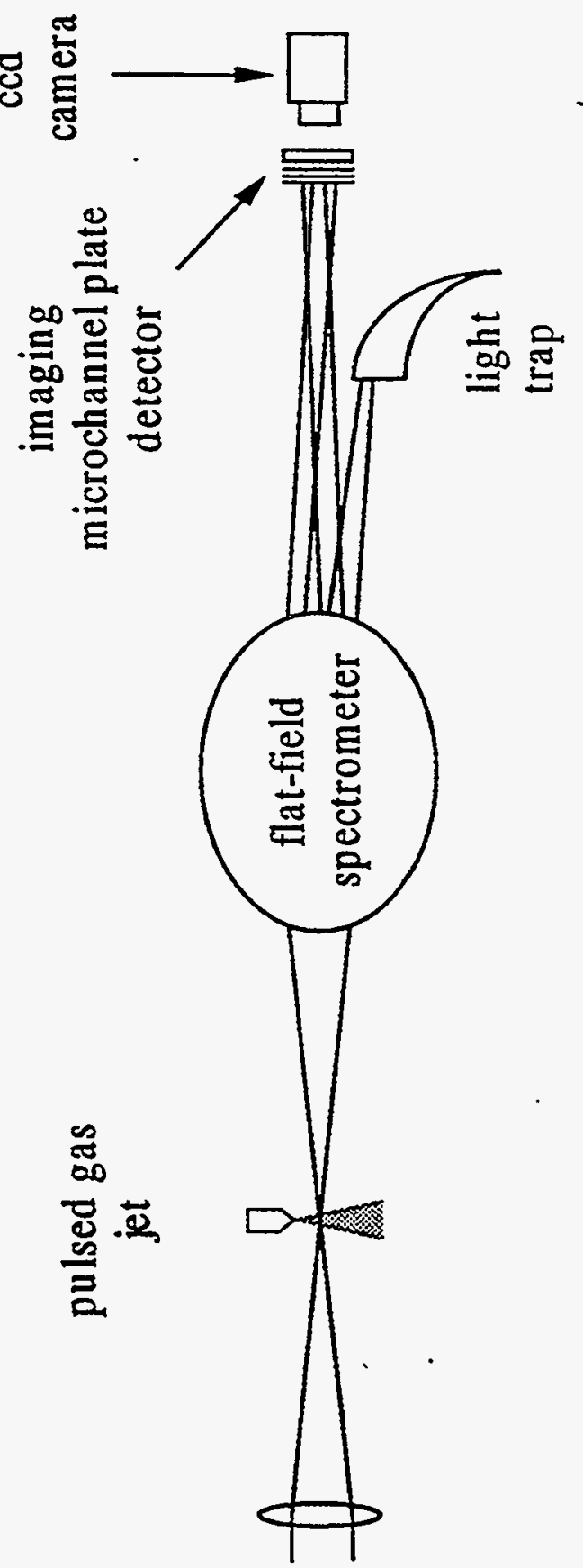

Figure 2.13: A block diagram of the experimental set-up required for optical harmonic generation. 
tube on the front of the vacuum chamber which ensures that the vacuum window is never more than a few centimeters from the focusing lens. This is particularly important for the Cr:LiSAF system where self-phase modulation in air might cause distortion of the beam due to the high power density being propagated. The lens is placed such that its focus is located directly beneath the output of a pulsed gas jet. Two types of pulsed gas jets were employed, one utilizing a solenoid valve to control the duration of the gas pulse and the other a piezoelectric crystal. In either case the valve is opened for a duration of $\sim 250 \mu \mathrm{sec}$ and produces a sonic expansion of gas with a density of $\sim 10^{17}$ particles $/ \mathrm{cm}^{3}$. The exact density achieved is a function of the backing pressure applied.[perry92b]

After the gas jet, the beam diverges into a monochromator to spectrally disperse the harmonics. Two different dispersing systems were utilized. For the preliminary experiments a $.25 \mathrm{~m}$ Seya-Namioka monochromator (McPherson 234) was utilized. This set-up is shown in Figure 2.14. In this device the cone of radiation is incident on a variable width entrànce slit, typically $\sim 100 \mu \mathrm{m}$ wide. The light expands to fill a $1200 \mathrm{~g} / \mathrm{mm}$ iridium coated toroidal diffraction grating. The wavelength selected is refocused onto an exit slit and detected by a chevron configuration dual microchannel plate detector (Galileo TOF 3000). The microchannel plate signal is then sent into a gated integrator and the integrated signal is sent via an analog-to-digital converter to a PC.

In the second system, shown in Figure 2.15, a slitless spectrometer directly images the focal position of the laser onto the exit slit and an electron multiplier is used to detect the signal.[lhuil93] All of the light leaving the focus is collected by a gold-coated toroidal mirror with a $1 \mathrm{~m}$ focal length and sent onto a gold-coated variable groove density plane diffraction grating with average groove spacing of 1700 grooves $/ \mathrm{mm}$. After spectral dispersion the residual fundamental laser radiation is picked off by a conical glass light trap to minimize scattered light problems. This device may be operated as either a spectrometer, where large portions of the spectrum may be recorded 
$1200 \mathrm{groove} / \mathrm{mm}$

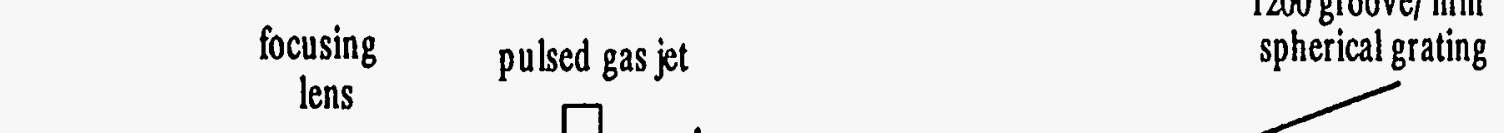

㯈

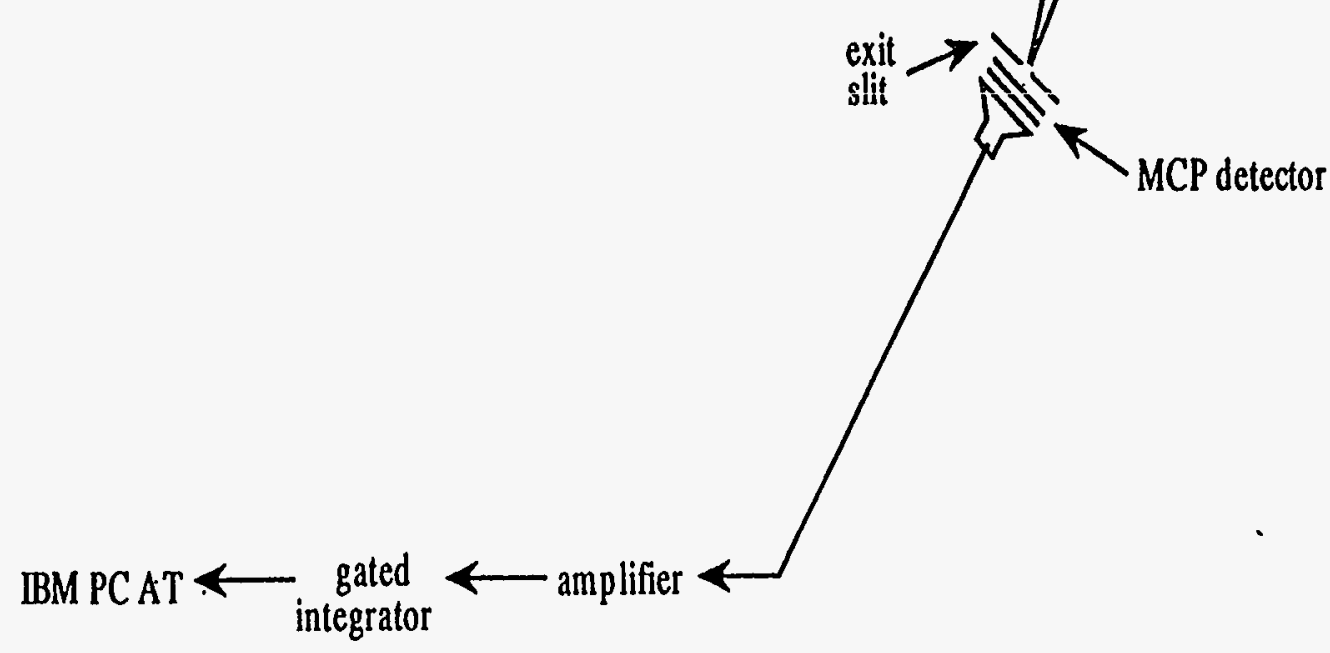




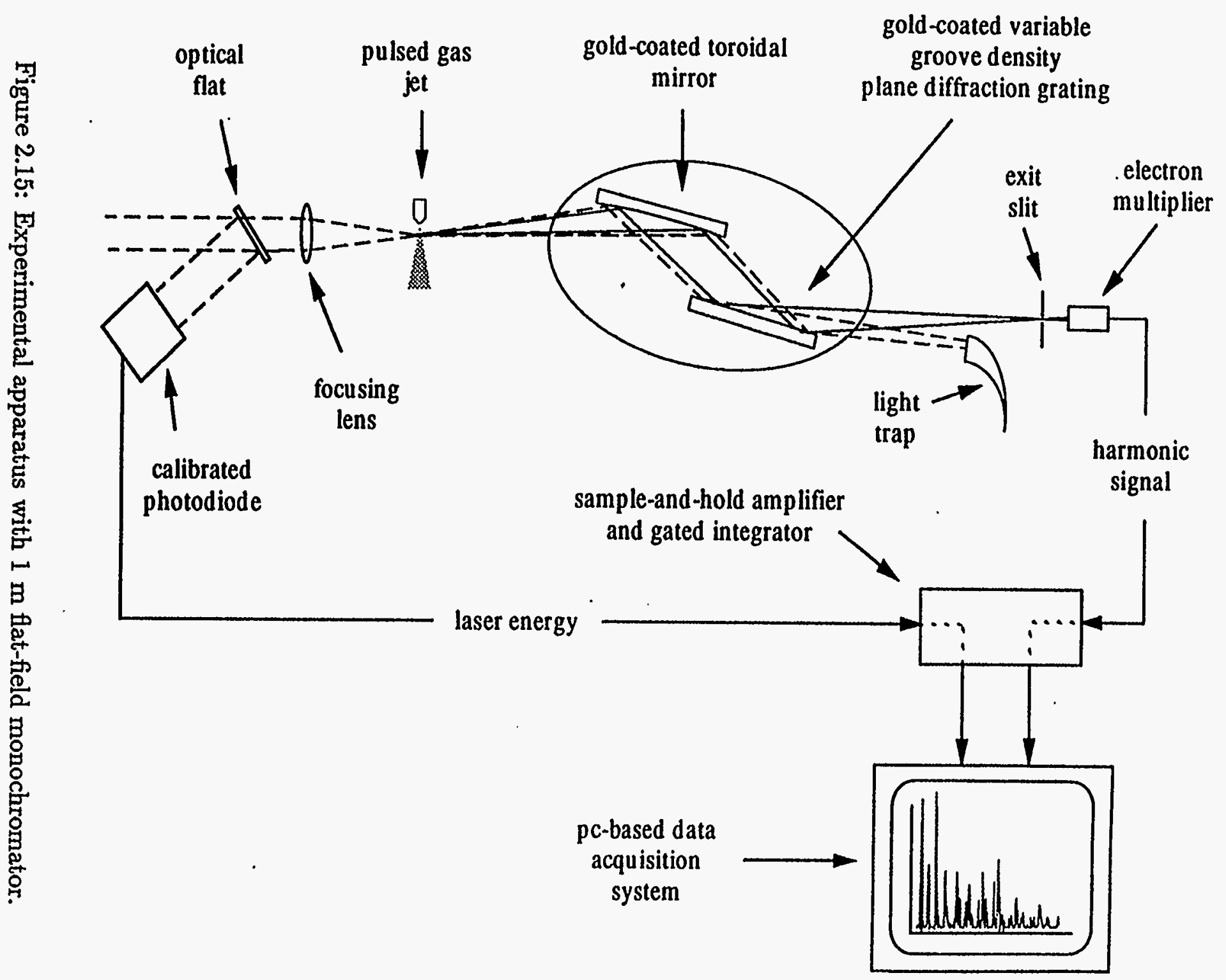

त्र 
on each shot, or as a monochromator where an exit slit is put in place and one wavelength at a time is recorded and the grating is then moved to the next wavelength. The use of a plane, variable groove density diffraction grating ensures that the dispersed wavelengths are imaged onto a plane rather than the Rowland circle, enabling detection of several wavelengths simultaneously with standard planar detectors. This distinction is very important for the spatial measurements presented in Chapter 5 since it makes use of large area imaging detectors relatively straightforward. This spectrometer was absolutely calibrated for efficiency at a synchrotron source[lhuil9.3] so the absolute number of photons detected can be determined.

For experiments requiring the total number of harmonic photons produced an electron multiplier is used to detect the harmonic signal. This signal is integrated and read out to a PC along with the laser energy for the shot. The data acquisition system controls the position of the grating allowing for the recording of harmonic signal as a function of wavelength, intensity, or "position", where "position" can refer to any parameter to be varied. When the spatial distributions of the harmonics are being studied, the exit slit of the monochromator is removed and an imaging microchannel plate detector is placed $\sim 13 \mathrm{~cm}$ beyond the focal plane of the spectrometer. This is done to allow the harmonics to reexpand and better fill the detector so small scale structures will be detectable. The imaging detector consists of two $10 \mu \mathrm{m}$ pore microchannel plates mounted in chevron configuration with the top plate coated with CsI to increase its efficiency. The electrons leaving the back channel plate illuminate a phosphor screen. A ccd camera is used to record the image off of the phosphor screen and the image is recorded on a PC. 


\section{Chapter 3}

\section{Optical Harmonic Generation}

\section{1 "Weak" Field Harmonic Generation}

Doing several preliminary experiments in the weak field regime is an opportunity to characterize the experimental set-up and ensure that the properties of each element are well understood. This will minimize the amount of experimental uncertainty present when studying the process in the more complicated, "strong" field regime.

In Chapter 1 , an analytic solution was derived for the intensity of the qth harmonic as a function of the incident laser intensity with the form

$$
\mathrm{I}_{q} \propto\left|\chi^{(q)}\right|^{2} N^{2} \mathrm{I}_{1 \omega}^{q}|F(b \Delta k)|^{2}
$$

where $\chi^{(q)}$ is the $q$ th order nonlinear susceptibility, $N$ is the atomic density, $F(b \Delta k)$ is the phase matching integral, and $I_{1 \omega}$ is the incident laser intensity.

By verifying the density dependence predicted by perturbation theory the coherent nature of the process of harmonic generation can also be verified. To show that the $N^{2}$ dependence is a characteristic of a coherent process, consider the problem of a collection of radiating oscillators as illustrated in Figure 3.1. Each of the oscillators produces an electric field of the form

$$
\mathrm{E}_{i}=\mathrm{E}_{o i} e^{i\left(\overrightarrow{\left.k_{i} \cdot \vec{r}-\omega_{i} t+\varphi_{i}\right)}\right.}=\mathrm{E}_{o i} e^{i \phi_{i}}
$$




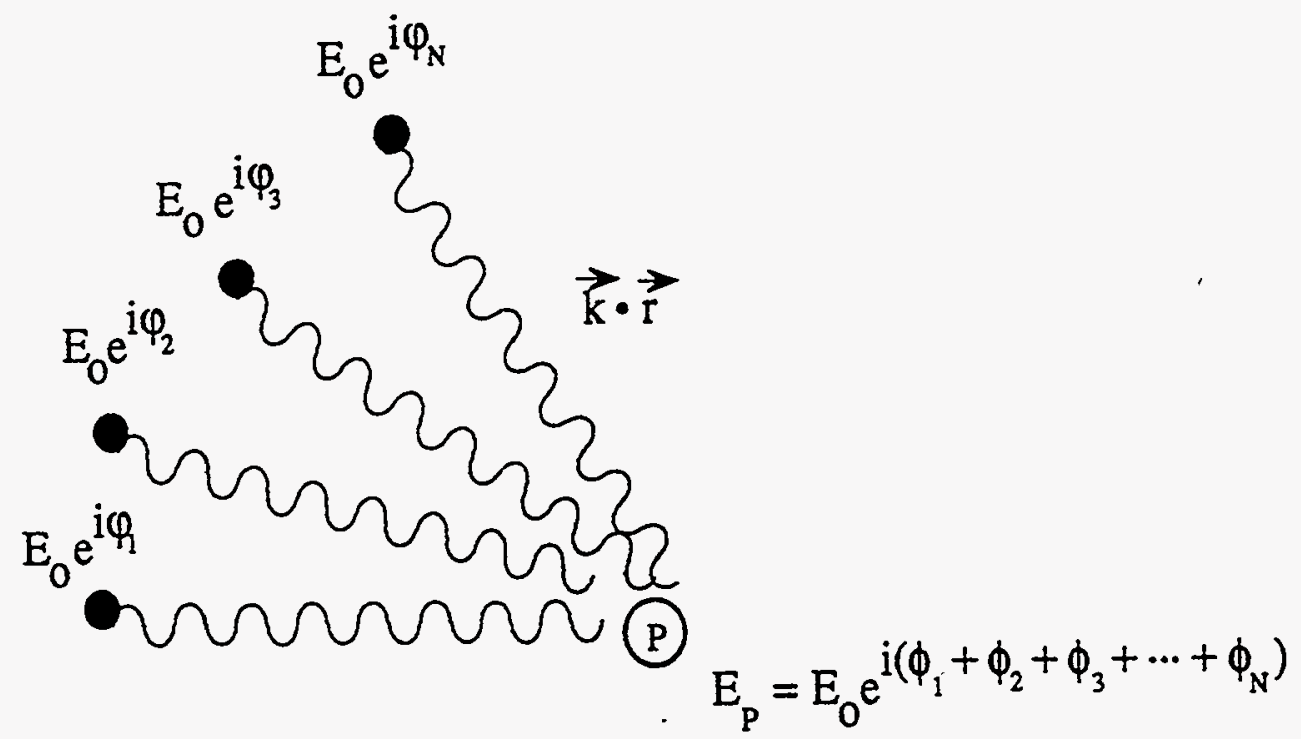

Figure 3.1: Illustration of the problem of determing the field at point $\mathrm{P}$ due to a collection of radiating oscillators.

where $\mathrm{E}_{o i}$ is the time-independent amplitude of the field. The total field at point $\mathrm{P}$ is then

$$
E_{\text {total }}=\sum_{i} E_{i} \text {. }
$$

If the field amplitudes are assumed to be equal this becomes

$$
\mathrm{E}_{\mathrm{total}}=\mathrm{E}_{o}\left(e^{i \phi_{1}}+e^{i \phi_{2}}+\cdots+e^{i \phi_{N}}\right)
$$

The intensity at point $\mathrm{P}$ is

$$
\mathrm{I}_{\text {total }}=\frac{c n}{8 \pi}<\mathrm{E}_{\text {total }}^{*} \mathrm{E}_{\text {total }}>
$$

Now, if the fields are in phase at the point, $\mathrm{P}, \phi_{1}=\phi_{2}=\cdots=\phi_{N}$ and 3.4 becomes

$$
\mathrm{E}_{\text {total }}=N \cdot \mathrm{E}_{o} e^{i \phi}
$$

which yields

$$
\mathrm{I}_{\text {total }}=\frac{c n}{8 \pi} N^{2} \mathrm{E}_{o}^{2}
$$


However, for an incoherent process $\phi_{1} \neq \phi_{2} \neq \cdots \neq \phi_{N}$ and

$$
\begin{aligned}
\mathrm{E}_{\text {total }}^{*} \mathrm{E}_{\text {total }} & =\mathrm{E}_{o}^{2}\left(e^{-i \phi_{1}}+\cdots+e^{-i \phi_{N}}\right)\left(e^{i \phi_{1}}+\cdots+e^{i \phi_{N}}\right) \\
& =\mathrm{E}_{\circ}^{2} \sum_{i=1}^{N} \sum_{j=1}^{N} e^{i\left(\phi_{i}-\phi_{j}\right)} \\
& =\mathrm{E}_{o}^{2}\left[N+\sum_{i \neq j} 2 \cos \left(\phi_{i}-\phi_{j}\right)\right]
\end{aligned}
$$

so 3.5 becomes

$$
\begin{aligned}
I_{\text {total }} & =\frac{c n}{8 \pi}<E_{o}^{2}\left[N+\sum_{i \neq j} 2 \cos \left(\phi_{i}-\phi_{j}\right)\right]> \\
& =\frac{c n}{8 \pi} N E_{o}^{2} .
\end{aligned}
$$

It has been demonstrated that the density produced by the pulsed gas jet utilized for these experiments varies linearly with the backing pressure applied.[perry92b] Therefore, harmonic signal as a function of density may be directly probed by varying the backing pressure and the result is presented in Figure 3.2 where the solid line represents a fit of the form $\mathrm{I}_{3 \omega} \propto N^{2}$.

A simple experimental confirmation of the intensity dependence of harmonic generation is to examine the production of third harmonic in xenon using the second harmonic of the dye laser as the driver. The laser was operated at $580 \mathrm{~nm}$ ( $3 \mathrm{~mJ}$ ) and the fundamental was frequency doubled in a $1 \mathrm{~cm}$ long KDP crystal producing typically $300-900 \mu J$ at $290 \mathrm{~nm}$ resulting in an incident laser intensity $\sim .4-1 \times 10^{13} \mathrm{~W} / \mathrm{cm}^{2}$. A plot of third harmonic intensity as a function of incident laser intensity is shown in Figure 3.3 where the solid line is a fit of the form $\dot{I}_{3 \omega} \propto I_{1 \omega}^{3}$. The fit is in very good agreement with the data over the intensity range studied.

\subsection{Harmonic Generation in the "Strong" Field}

In order to study the production of very high harmonics, the laser intensity must be increased to the point where its strength begins to rival that of the atomic binding 


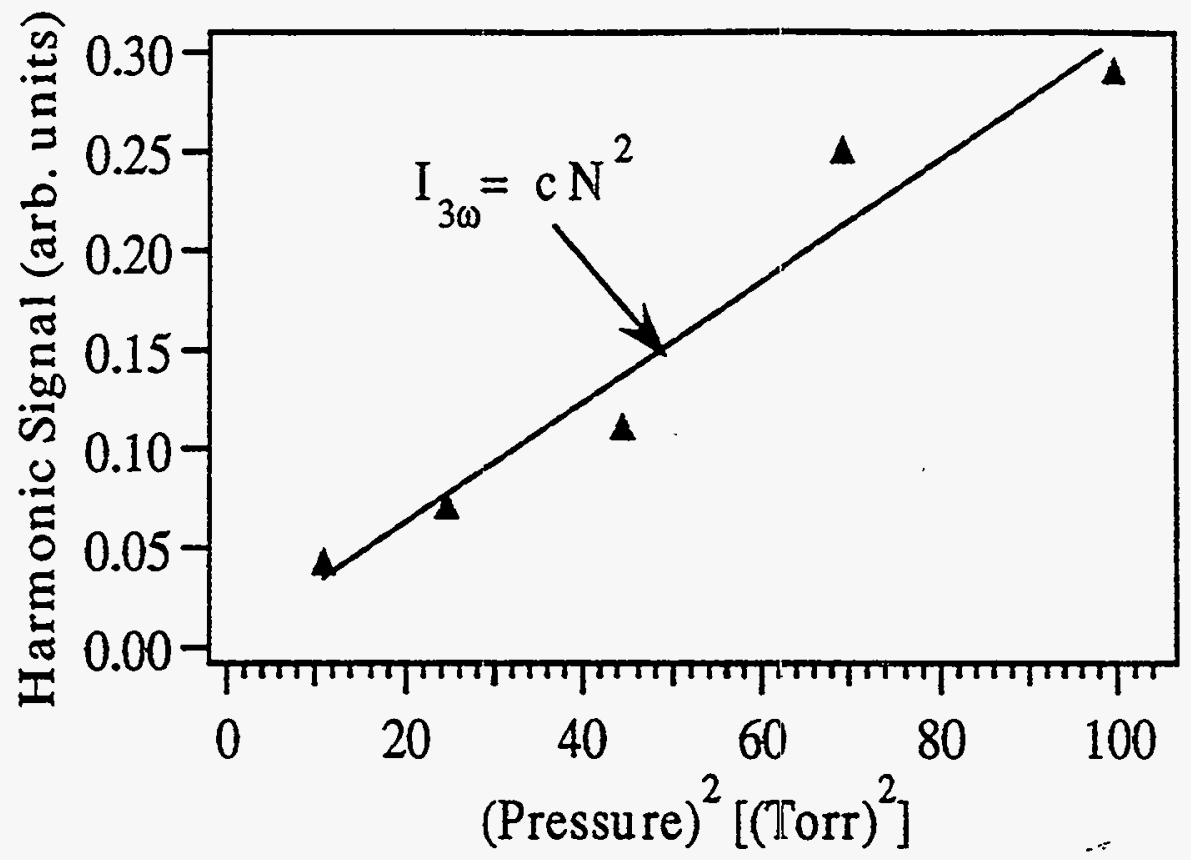

Figure 3.2: Third harmonic signal as a function of gas density for xenon at $~$ $10^{13} \mathrm{~W} / \mathrm{cm}^{2}$. The solid line shows an $N^{2}$ fit to the data.

field. When such an intense laser field is incident upon a dense gaseous medium, an extended plateau begins to form where each successive harmonic is generated with essentially the same efficiency. Figure 3.4 shows the spectrum measured by the flatfield monochromator previously described using the dye laser operating at $600 \mathrm{~nm}$ with xenon as the target gas. When the $1 \mathrm{~cm}$ beam is focused by a $30 \mathrm{~cm}$ lens ( $f^{\#}$ $=30$ ) and an atomic density of $\sim 20$ torr, the thirteenth harmonic is the shortest wavelength detected $(46.1 \mathrm{~nm})$. The maximum number of harmonic photons detected was $4 \times 10^{8}$ for the ninth harmonic, yielding a maximum conversion efficiency of $\sim 10^{-7}$.

As shown earlier, the position of the cutoff is determined by the incident laser intensity, its wavelength, and the ionization potential of the target species. Increasing the intensity is the most direct way to extend the plateau, although eventually the atom will ionize, the competing process to harmonic generation. Utilizing a longer wavelength driver also results in higher order harmonics in the plateau so the LiSAF 


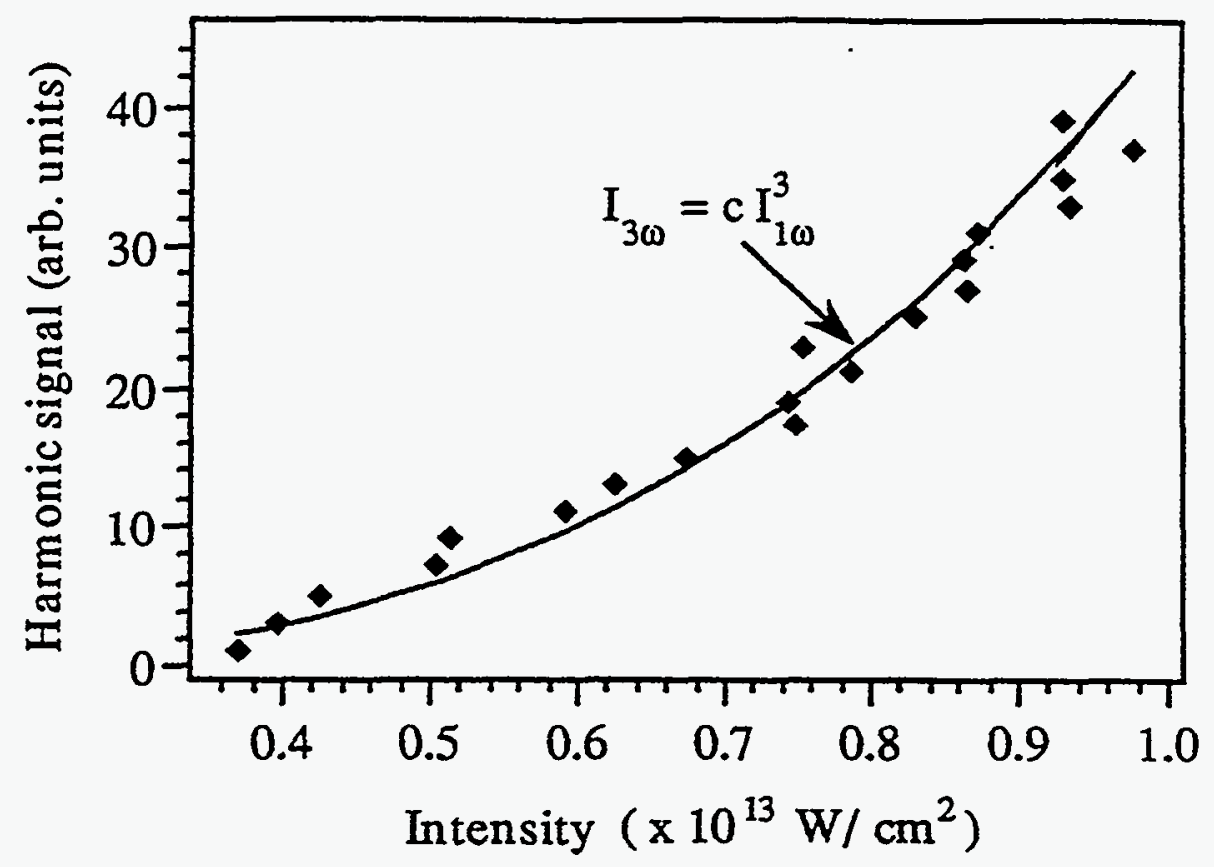

Figure 3.3: Third harmonic intensity as a function of incident laser intensity. The solid line is a least squares fit to the data.

laser operating at $825 \mathrm{~nm}$ is an attractive candidate. An $f^{\#}=24$ focusing system was utilized and the atomic density was typically $\sim 20$ torr. By switching to the LiSAF laser as the driver, the intensity can easily be increased to $>10^{14} \mathrm{~W} / \mathrm{cm}^{2}$ and in addition the shorter pulse duration raises the saturation intensity for ionization of the medium. A spectrum taken at $8 \times 10^{13} \mathrm{~W} / \mathrm{cm}^{2}$ in xenon is presented in Figure 3.5. Here the incident photon flux is $\sim 8 \times 10^{15}$ photons per pulse. The maximum number of photons detected was $3.5 \times 10^{9}$ at the thirteenth harmonic for a conversion efficiency of $4 \times 10^{-7}$, similar to that achieved with the dye laser. Xenon, since it is readily polarizable, provides very good conversion efficiency but is limited to relatively low order processes due to its low ionization potential $(12.1 \mathrm{eV})$.

The final option explored for extending the plateau observed is to use a target gas with a higher ionization potential, such as argon with a binding energy of $15.6 \mathrm{eV}$. Figure 3.6 shows the twenty-first through thirty-first harmonics produced in argon with the LiSAF laser, a dramatic improvement over the spectrum observed in xenon. 


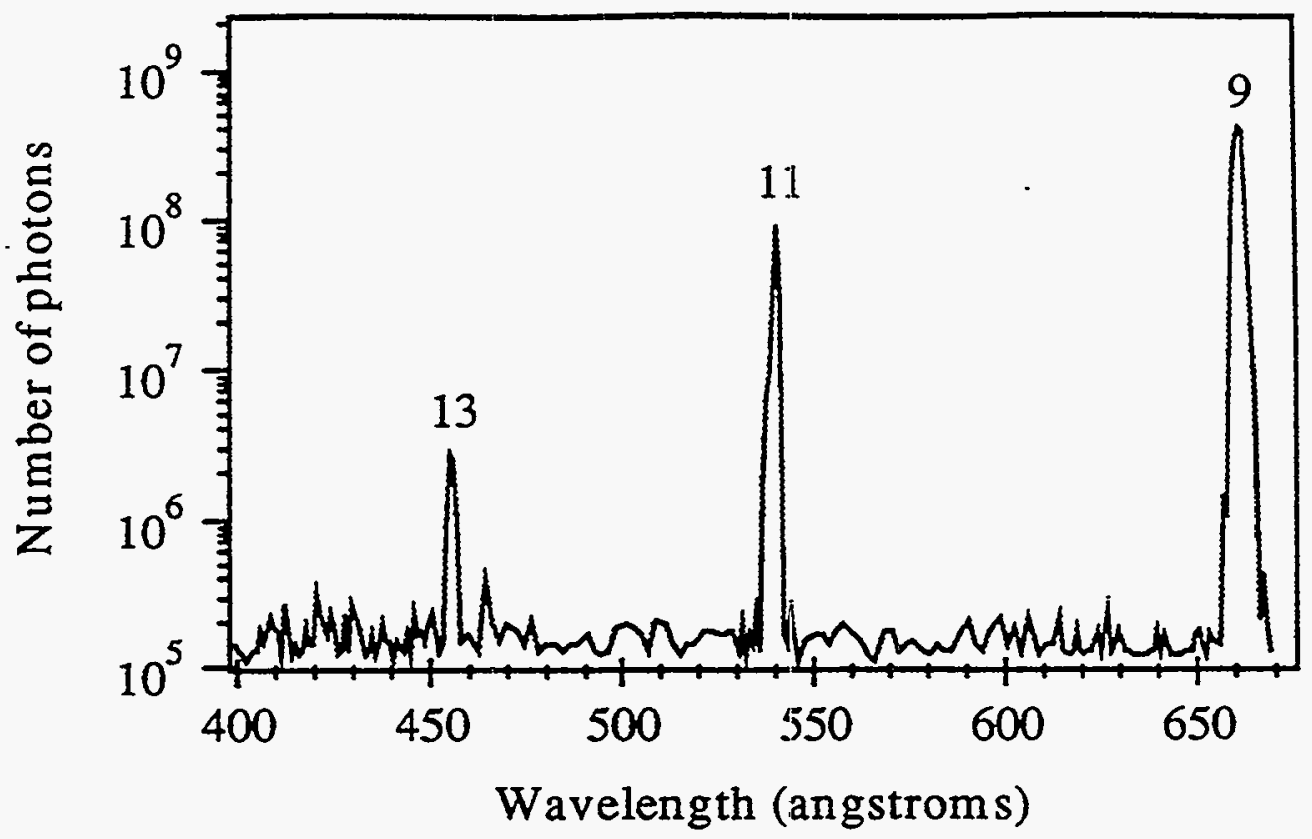

Figure 3.4: Harmonic spectrum produced in xenon with the dye laser operating at $600 \mathrm{~nm}$ as the driver. The intensity is $6 \times 10^{13} \mathrm{~W} / \mathrm{cm}^{2}$.

At an intensity of $3.5 \times 10^{14} \mathrm{~W} / \mathrm{cm}^{2}$ the highest order observable was thirty-third, corresponding to a wavelength of $25.0 \mathrm{~nm}$. Here the conversion efficiency is $\sim 10^{-8}$, slightly lower than when xenon is utilized. The higher ionization potential of the atom (15.6 eV for argon compared to 12.1 for xenon), is indicative of it being more tightly bound and thus more difficult to polarize, lowering the conversion efficiency slightly overall.

The final gas studied was neon which has an ionization potential of $21.6 \mathrm{eV}$. Figure 3.7 shows a spectrum obtained in neon with the LiSAF driver. The highest observable harmonic in neon was the eighty-fifth, corresponding to a wavelength of $9.7 \mathrm{~nm}$. Much lower conversion efficiencies are observed corresponding to a peak of $\sim 4 \times 10^{-10}$ for $6 \times 10^{6}$ harmonic photons detected at the twenty-third harmonic with $50 \mathrm{~mJ}$ incident laser energy and an $f^{\#}=24$ focusing geometry. Utilizing higher energy drivers and larger focal volumes could readily increase this efficiency. Neon (or helium with an ionization potential of $24.6 \mathrm{eV}$ ) is the most attractive candidate for extremely short 


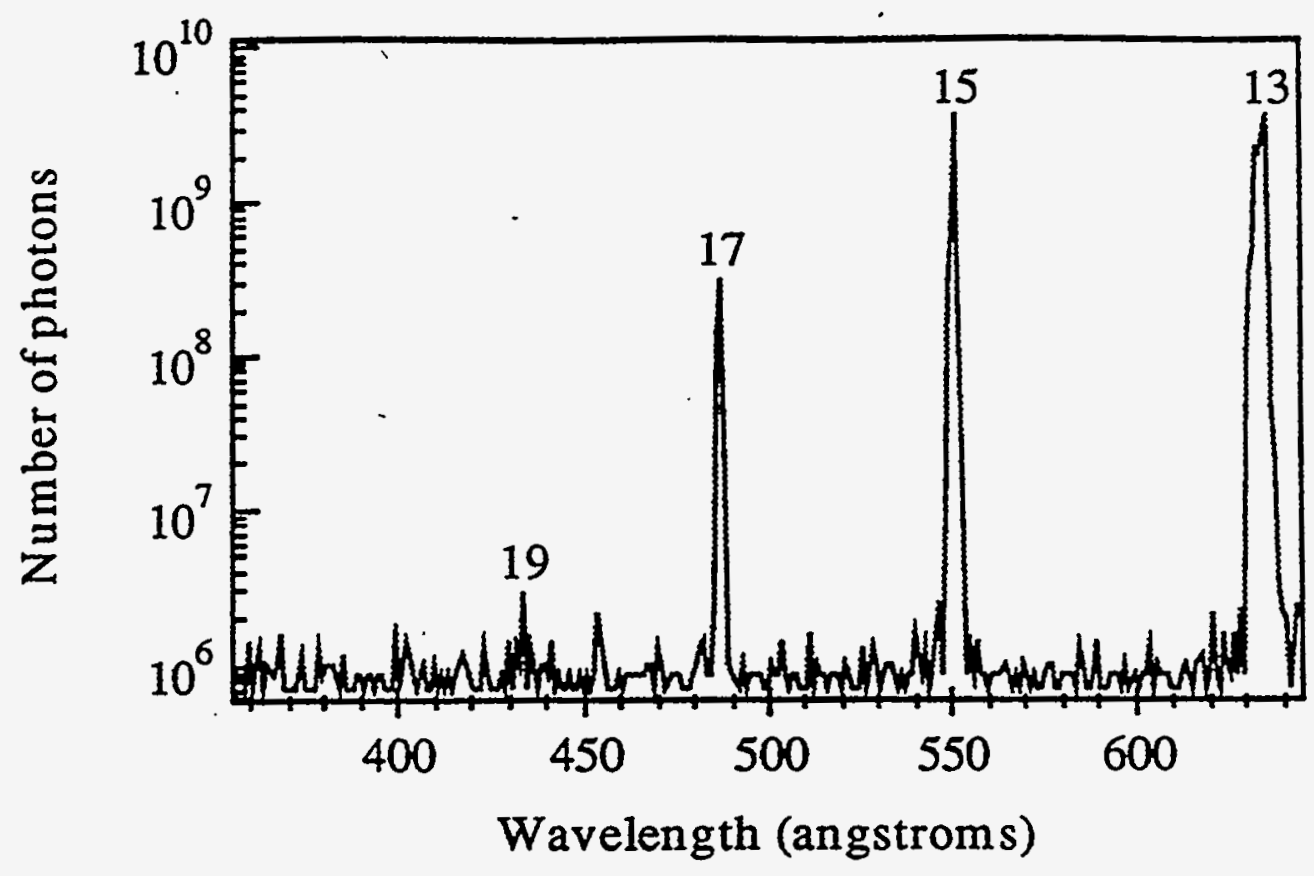

Figure 3.5: Harmonic spectrum produced in xenon at $8 \times 10^{13} \mathrm{~W} / \mathrm{cm}^{2}$ with the LiSAF laser.

wavelength production.

A plot of the intensity dependence of the twenty-fifth harmonic driven by the LiSAF laser as a function of the incident laser intensity is presented in Figure 3.8. Three distinct slopes are discernible which can be assigned as follows. In the first region, the harmonic is detected at its appearance intensity and exhibits a very steep dependence on the laser intensity $\left(I_{25 \omega} \sim I_{1 \omega}^{13}\right)$ corresponding to it being positioned in the cutoff of the harmonic spectrum. In the second region the dependence is slightly weaker $\left(I_{25 \omega} \sim I_{1 \omega}^{9}\right)$ and the harmonic has joined the plateau. Finally, the atom begins to ionize and the signal ceases increasing with increasing intensity since the number of atoms available to generate harmonics is beginning to be depleted. By examining the intensity dependence of a given harmonic in each of these regions, an effective nonlinear order can be assigned to the process for a given intensity. In this case the effective order $p$ is $\sim 13$ in the cutoff and $\sim 9$ in the plateau as opposed to the actual process order $q \sim 25$ utilized in the LOPT expressions. By examining the position 


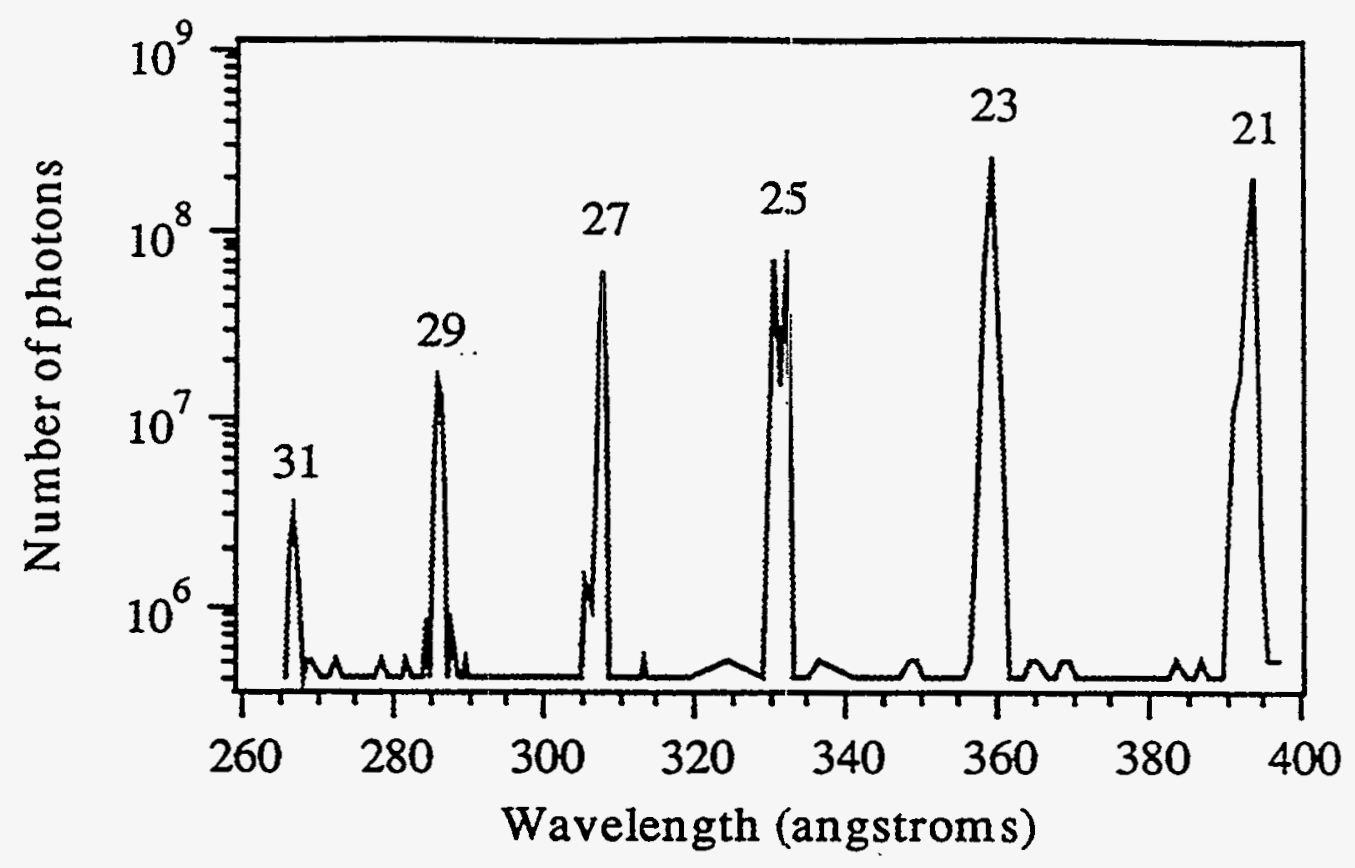

Figure 3.6: Harmonic spectrum obtained in argon with the LiSAF laser. The intensity is $\sim 1.1 \times 10^{14} \mathrm{~W} / \mathrm{cm}^{2}$. .

of saturation due to ionization, the saturation intensity of argon for $130 \mathrm{fsec}$ pulses can be estimated to be $\sim 3.5 \times 10^{14} \mathrm{~W} / \mathrm{cm}^{2}$. Since exactly calibrating the intensity at focus is extremely difficult an error of up to a factor of 2 can be assumed.

Figure 3.9 presents similar curves for the twenty-third, thirty-third, forty-third, fifty-third and sixty-third harmonics generated in neon with the LiSAF laser. In neon the behavior is very similar to that observed in argon. Three slopes are discernible with the steepest dependence occuring in the region just beyond the appearance intensity, the cutoff. The effective nonlinear order: of the process still exhibits a change from slightly greater than 10 in the cutoff to slightly lower than 10 in the plateau with minor variances from order to order. The saturation intensity is $\sim 8 \times 10^{14} \mathrm{~W} / \mathrm{cm}^{2}$. 


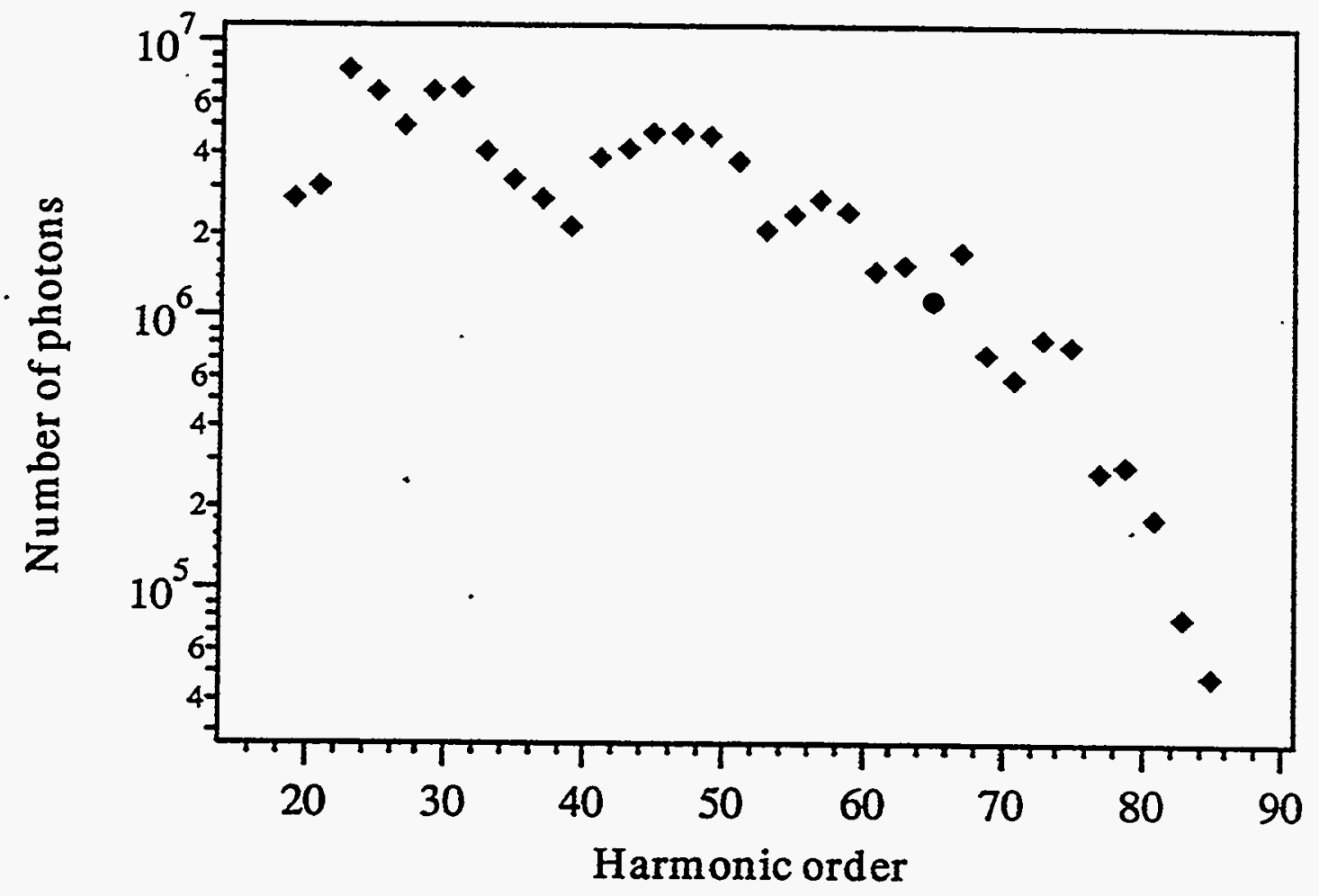

Figure 3.7: Section of a harmonic spectrum obtained in neon at an intensity of $3.3 \times$ $10^{14} \mathrm{~W} / \mathrm{cm}^{2}$. 


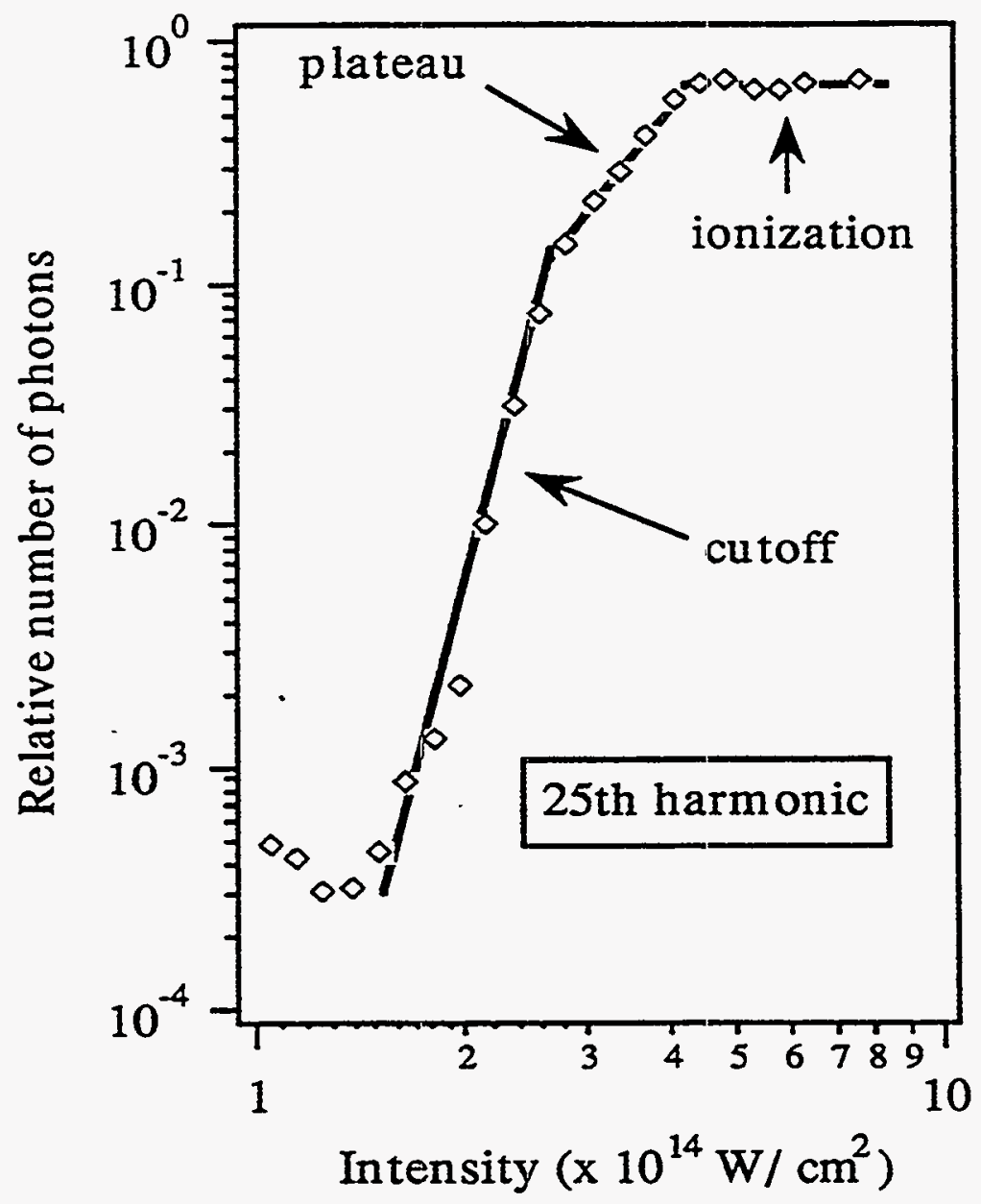

Figure 3.8: The relative number of photons at the twenty-fifth harmonic produced as a function of incident laser intensity for argon driven by the LiSAF laser. The solid lines are used to indicate the positions of changing slope of the curve. 

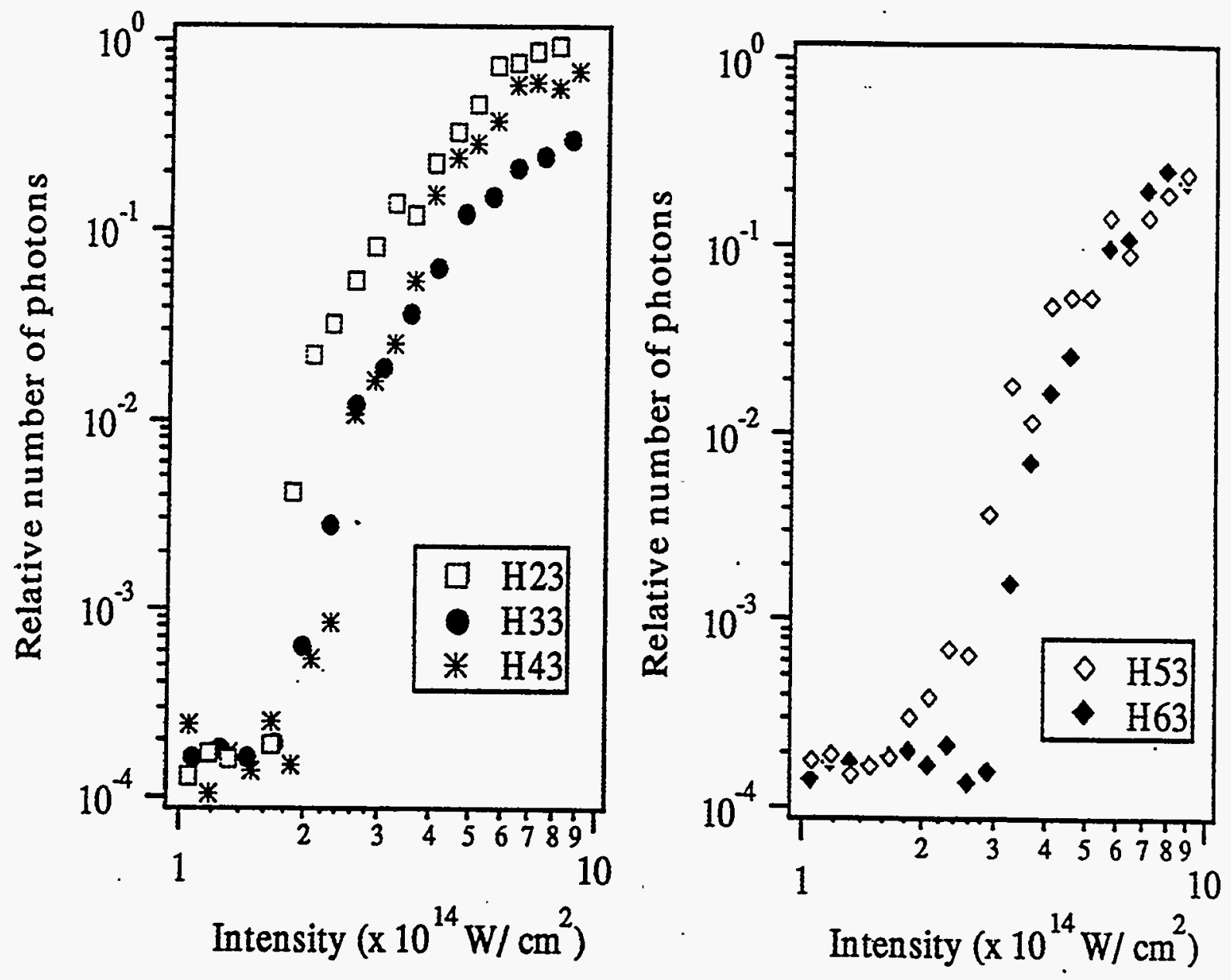

Figure 3.9: Relative number of harmonic photons produced as a function of the incident laser intensity for neon utilizing the LiSAF laser driver. 


\section{Chapter 4}

\section{The Two-Step Semiclassical Model}

\subsection{Theoretical Approach}

Theory efforts based on the solution of the time-dependent Schrödinger equation have provided a tremendious amount of insight into the physics underlying harmonic generation. It is, however, computationally complex and other alternatives have been explored. In an effort to provide a simple, intuitive model of harmonic generation, the so-called two-step semiclassical model has been proposed.[kulan93, schaf93, corku93] The process is divided into two steps, (1) ionization and (2) radiative recombination. The first step is envisaged as follows. The binding potential of the atom is distorted by the presence of the oscillating electric field as depicted in Figure 4.1. An electron can ionize by tunneling through the suppressed barrier [keldy65, ammos86, perry88, augst91] and the motion of the electron in the electric field of the laser is then studied classically.

The first step is to determine the probability that an electron will ionize at a given point in the laser field. The ionization rate for a. static dc field is given by

$$
W_{\mathrm{dc}}=4 \omega_{0}\left(\frac{\mathcal{E}}{\mathcal{E}_{\mathrm{H}}}\right)^{5 / 2} \frac{E_{\mathrm{a}}}{E(t)} \exp ^{\frac{-2}{3}\left(\frac{\mathcal{E}}{\mathcal{E}_{\mathrm{H}}}\right)^{3 / 2} \frac{E_{\mathrm{Z}}}{E_{(t)}}}
$$

where $\omega_{0}=4.1 \times 10^{16} \mathrm{sec}^{-1}$ is the atomic frequency, $\mathcal{E}$ is the ionization potential of the atom in question, $\mathcal{E}_{\mathrm{H}}=13.6 \mathrm{eV}$, the ionization potential of hydrogen, $E_{\mathrm{a}}=$ 


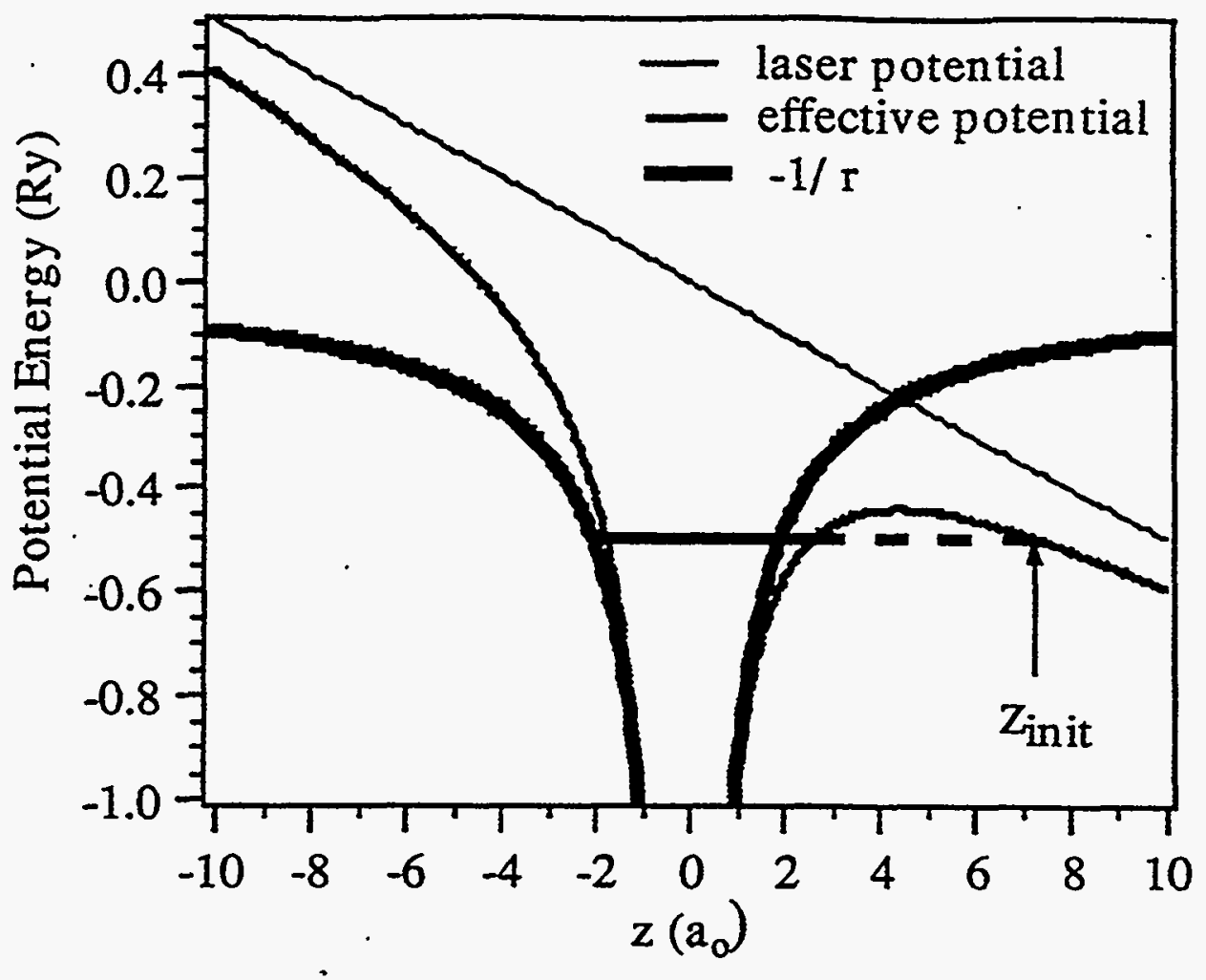

Figure 4.1: Schematic of the unperturbed potential for a hydrogen atom as well as the effective potential created by the influence of the laser field. The solid line within the potential well denotes the ground state wave function which can ionize by tunneling through the suppressed barrier.

$5.1 \times 10^{11} \mathrm{~V} / \mathrm{m}$ is the atomic field strength, and $E(t)$ is the laser field at time $t$. The ionization rate of helium for a laser field with a peak intensity of $5 \times 10^{14} \mathrm{~W} / \mathrm{cm}^{2}$ is shown in Figure 4.2.

Once the electron has ionized it will be treated as a free particle moving under the influence of the oscillating electric field and the atomic potential, and the classical equations of motion will be solved. The trajectories of electrons born during the first half of one laser cycle will be followed for 10 optical cycles of the laser field. Electrons born in the second half of the cycle will be identical except that their trajectories will be oppositely directed to those of the first half since the field will be identical but reversed in direction. Since harmonic radiation is produced when the orbiting electron is driven periodically across the atomic potential, only trajectories which return to the 


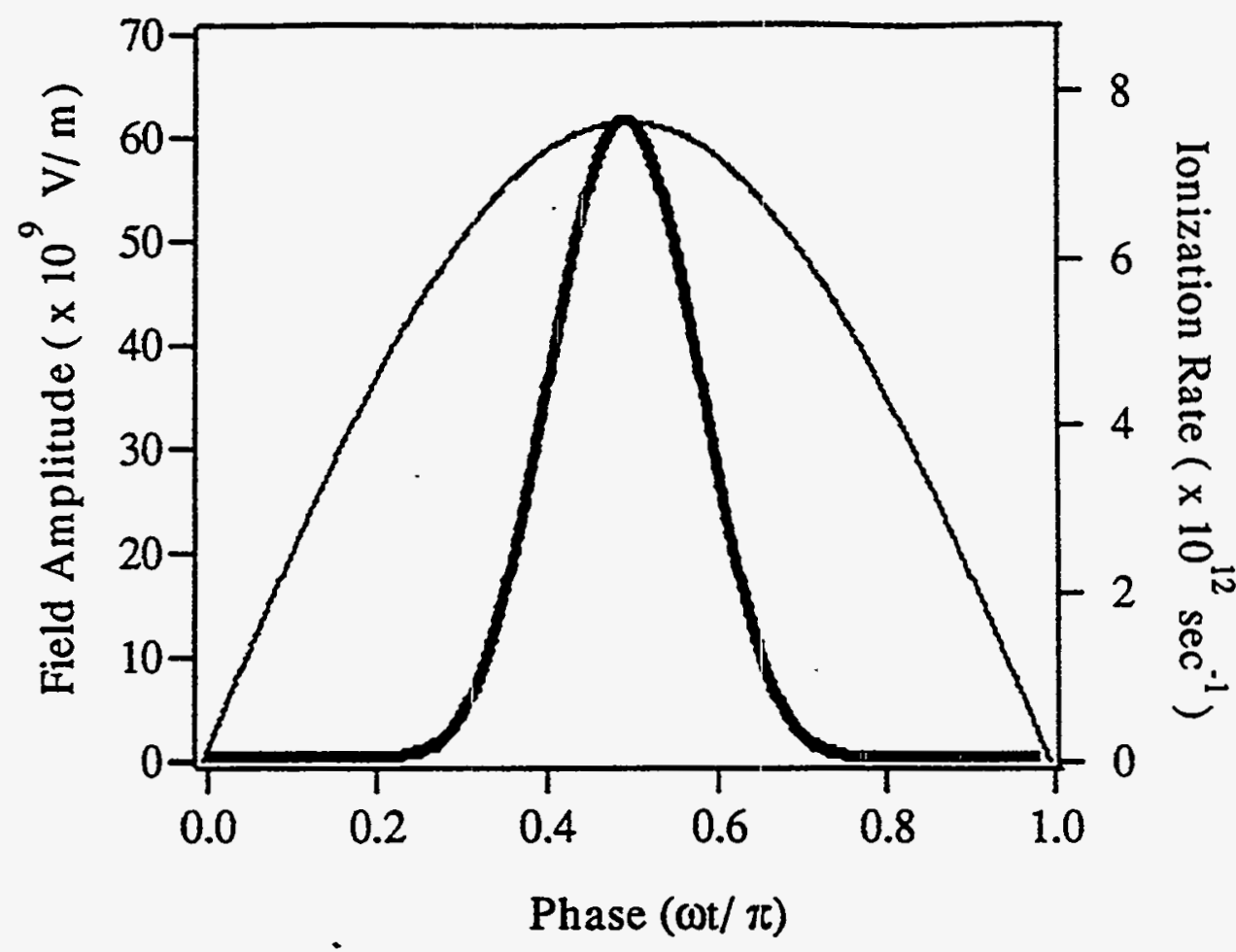

Figure 4.2: Ionization rate of helium (thick curve) as a function of the phase of the incident laser field (thin curve). The peak intensity of the laser field is $5 \times 10^{14} \mathrm{~W} / \mathrm{cm}^{2}$.

nucleus need be considered. Trajectories which do not return correspond to electrons ionizing. The electron's excursion from the nucleus can be very large, $x \gg a_{0}$, while the position at which it is born will be on the order of a few Bohr radii near the peak of the laser field. Therefore, the electron will be assumed to be born at $x \sim 0$.

Once the electron is born its motion is that of a free electron in an electric field where the force on it is given by

$$
\mathbf{F}(t)=m \mathbf{a}(t)=e \mathbf{E}(t)+e \mathbf{v}(t) \times \mathbf{B}(t)-\nabla \mathrm{V}_{\text {atomic }}
$$

where the $\mathbf{v} \times \mathbf{B}$ term is negligible in the nonrelativistic limit, $v \ll c$, and $-\nabla V_{\text {atomic }}$ is the force due to the atomic potential. If the influence of the atomic potential is included, this equation cannot be solved analytically. However, as a first approximation this influence may be neglected and the impact of this approximation 
will be examined. Using this approximation and applying an electric field given by $\mathrm{E}(t)=\mathrm{E}_{o} \sin (\omega t)$ polarized along the $\mathrm{x}$-axis yields,

$$
a_{x}(t)=\frac{e \mathrm{E}_{o}}{m} \sin (\omega t)
$$

subject to the initial condition that the electron is born at $x=0$ at $t=t_{o}$. Equation 4.3 can be readily integrated giving,

$$
v_{x}(t)=\frac{-e \mathrm{E}_{o}}{m \omega}\left[\cos (\omega t)-\cos \left(\omega t_{o}\right)\right]
$$

and

$$
x(t)=\frac{-e \mathrm{E}_{o}}{m \omega^{2}}\left[\sin (\omega t)-\sin \left(\omega t_{o}\right)-\omega\left(t-t_{o}\right) \cos \left(\omega t_{o}\right)\right] .
$$

The instantaneous kinetic energy of the electron is then given by

$$
\mathrm{K}(t)=\frac{1}{2} m \mathrm{v} \cdot \mathrm{v}=2 \mathrm{U}_{\mathrm{P}}\left[\cos (\omega t)-\cos \left(\omega t_{0}\right)\right]^{2}
$$

where $U_{P}=e^{2} \mathrm{E}_{o}^{2} / 4 m \omega^{2}$, is the ponderomotive potential or "quiver" energy of the electron in the field.

This method may be readily applied to calculating the harmonic spectrum for a given incident laser field. First, the ionization probability is calculated as a function of the phase of the laser field over the first half of one optical cycle for approximately five hundred phases. For each phase the electron trajectory is followed over ten cycles of the optical field and tested to see if it returns to the nucleus $(x(t)=0)$. A phase space plot of a representative trajectory is shown in Figure 4.3.

It is interesting to examine the energy of the electron each time it crosses the nucleus (or ion core). Figure 4.4 shows the energy of the electron at the time of return as a function of the phase of the field at which the electron was born. In the case where the influence of the atomic potential has been neglected the maximum kinetic energy of the electron corresponds to $3.17 U_{P}$, the value predicted by the empirical cut-off law, and no trajectories from before $\omega t_{o}=\pi / 2$ return to the vicinity of the nucleus. Past this maximum in the energy, all trajectories return only once and the energy trails off smoothly toward zero. Since the atomic potential is not included in this calculation 


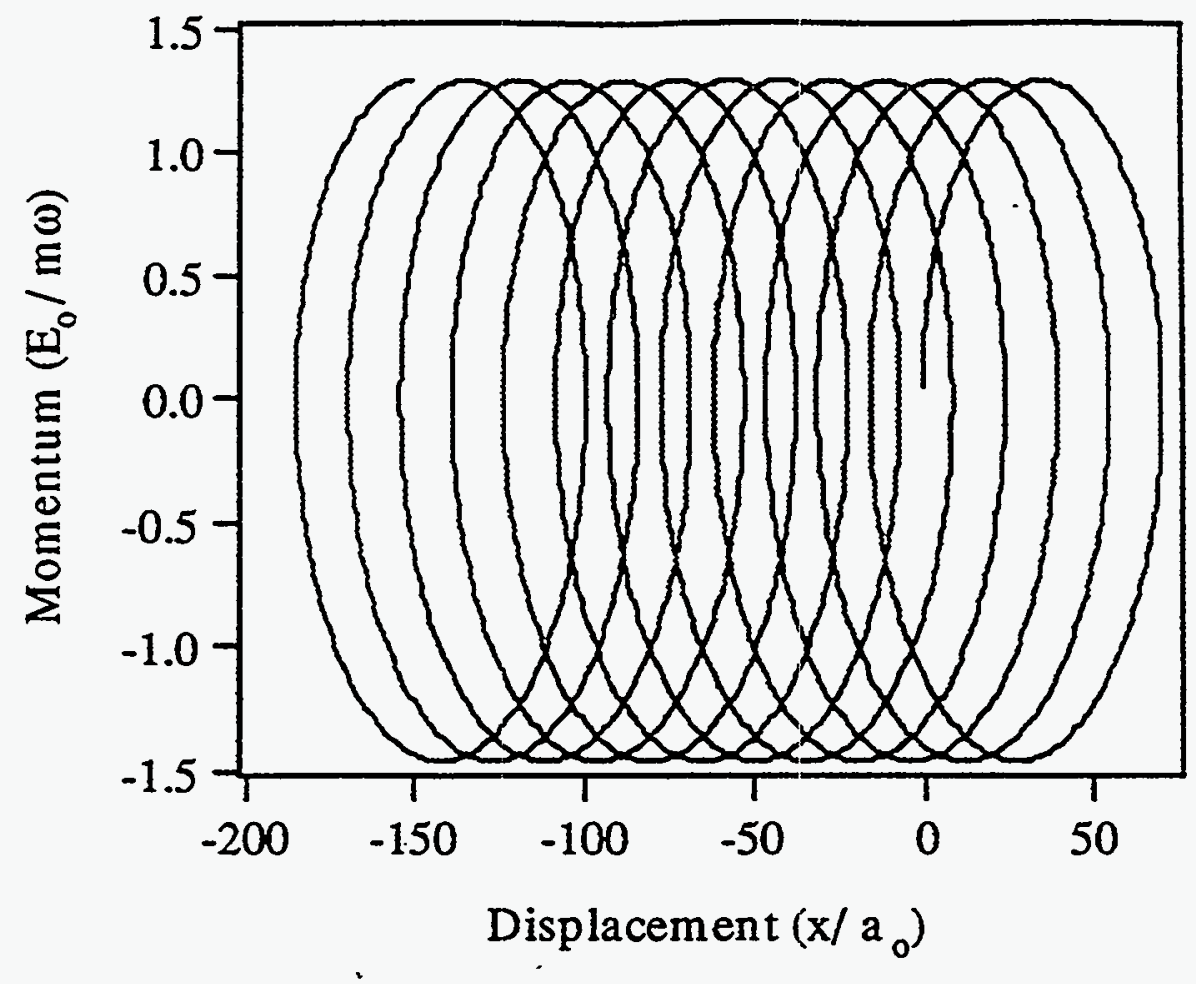

Figure 4.3: Phase space plot of an electron trajectory for phase $\omega t_{o}=1.571$ radians.

the factor of $I_{P}$ does not appear. The maximum energy occurs at $18^{\circ}$ past $\omega t_{0}=\pi / 2$, the peak of the incident laser field. In the calculation where the influence of the atomic potential is included, essentially the same plot is obtained although now trajectories before $\omega t_{o}=\pi / 2$ do return. The major difference occurs after the electron energy has peaked at $3.17 \mathrm{U}_{\mathrm{P}}$. The energy no longer simply decreases but rather begins to increase again past $\omega t_{0} \simeq 3 \pi / 4$ reaching a maximum of $8 \mathrm{U}_{\mathrm{P}}$. However, this significant deviation in behavior occurs in a region of negligible ionization probability so these trajectories will not influence the electron energy spectrum.

A harmonic "spectrum" can be generated by producing a histogram of collision probabilities as a function of electron energy. This continuous electron energy distribution is the analog of a photon energy distribution. Each time the trajectory crosses the nucleus, the kinetic energy of the electron at the time of the "return" is calculated and given a weight corresponding to the ionization probability for this phase and put 
(a)

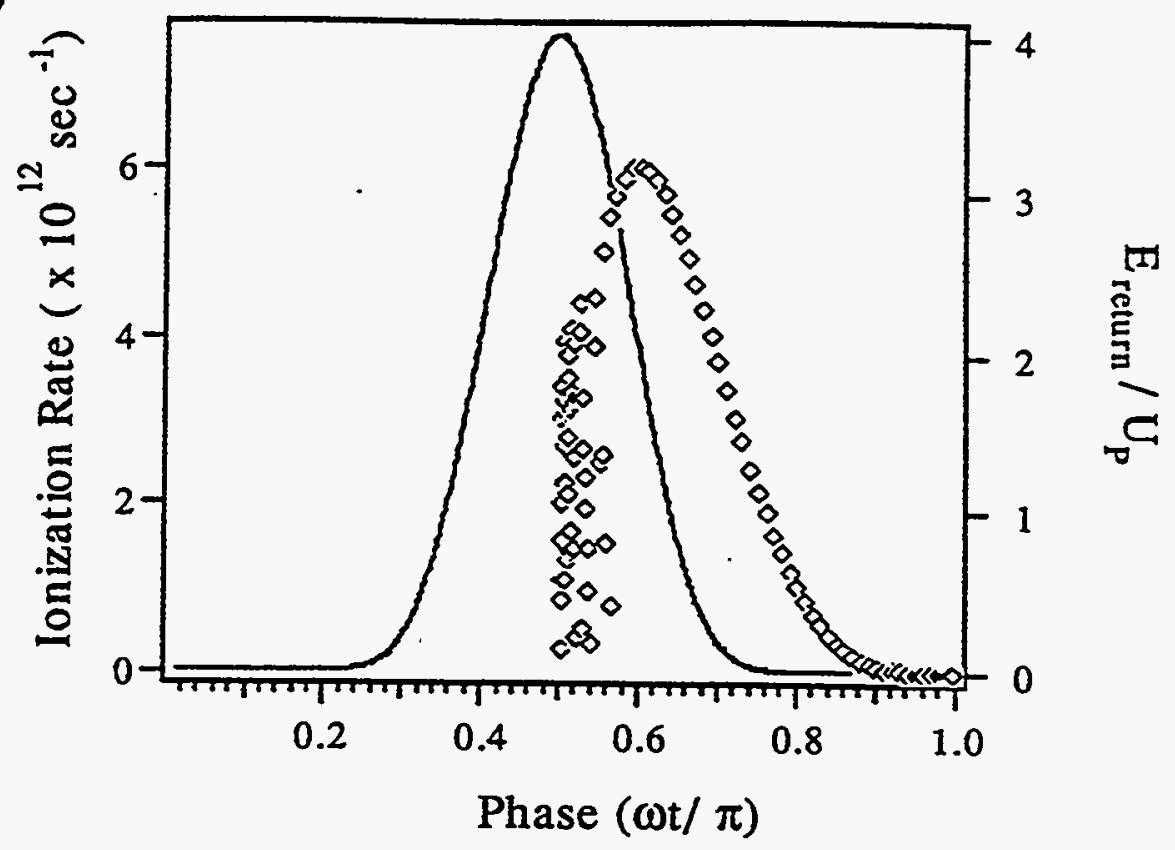

(b)

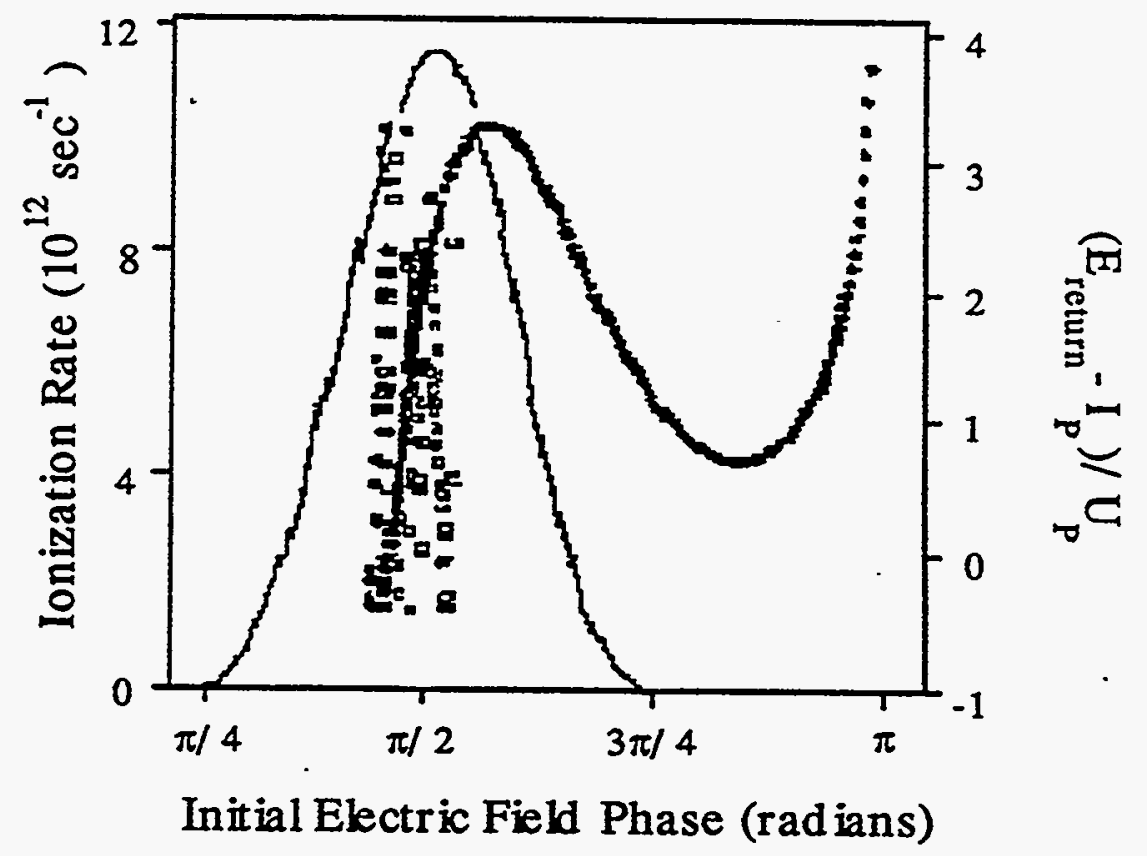

Figure 4.4: The energy of the electron upon return to the nucleus as a function of the phase of the laser electric field at which the electron was born (a) without the influence of the atomic potential included and (b) with the influence of the atomic potential. 
into a corresponding energy bin. After each return the electron is allowed to continue along its trajectory until five optical cycles of the laser field have been completed. The total weight in each energy bin is summed and plotted versus the energy of that bin. The probability for emitting a photon at each return is proportional to the dipole matrix element for the transition back to the ground state at the return energy of the electron. In the case of hydrogen at these energies, the dipole matrix element is decreasing by approximately $\omega^{-3}$.[bethe77] This effect is illustrated in Figure 4.5. The algorithm for these calculations is summarized below.

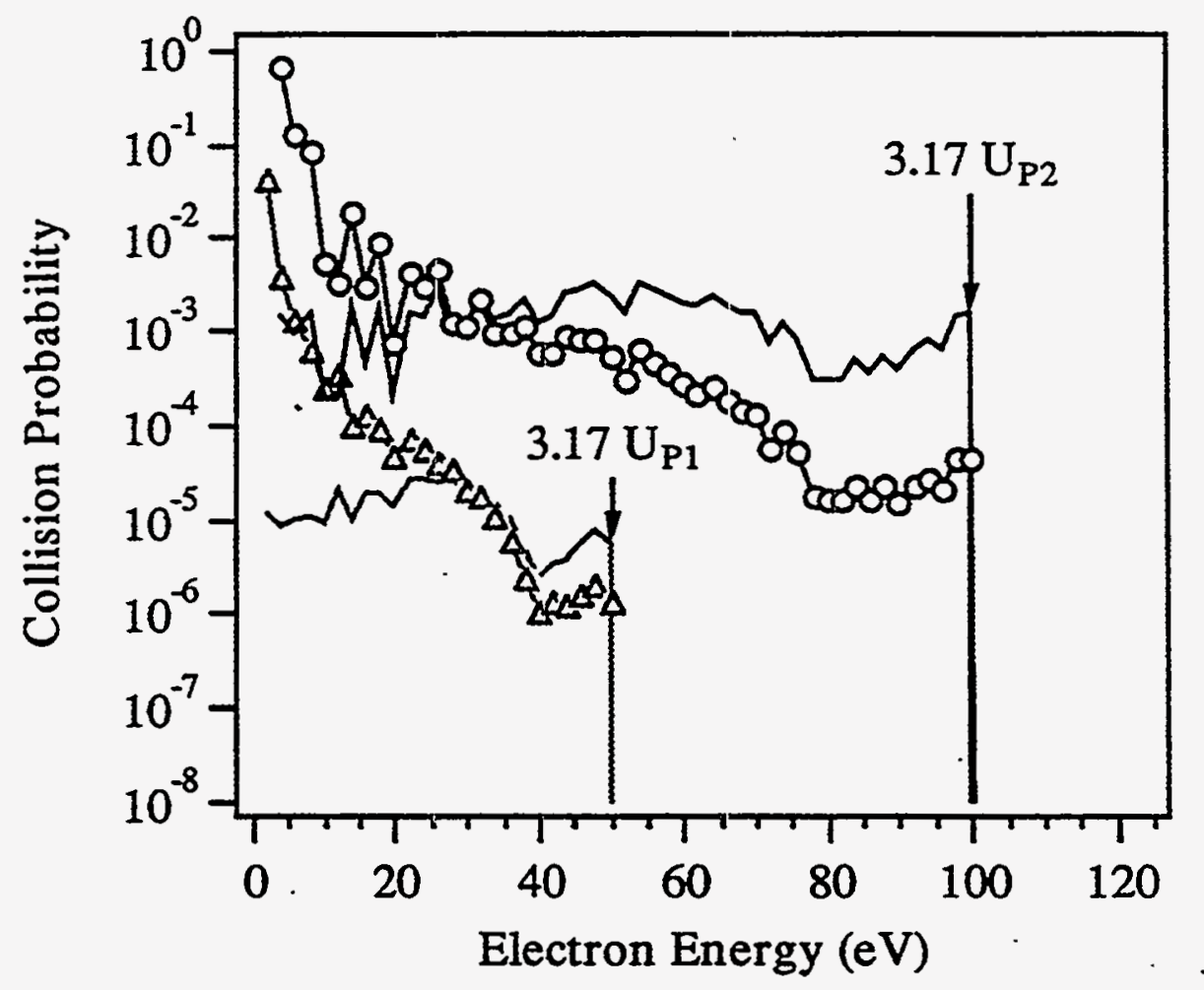

Figure 4.5: Energy histogram for uncorrected returns (solid line) and weighted returns with the $\omega^{-3}$ dependence of the oscillator strength included (open symbols) for two intensities. $\mathrm{I}_{1}=2.5 \times 10^{14} \mathrm{~W} / \mathrm{cm}^{2}, \mathrm{U}_{\mathrm{P} 1}=15.8 \mathrm{eV} ; \mathrm{I}_{2}=5.0 \times 10^{14} \mathrm{~W} / \mathrm{cm}^{2}, \mathrm{U}_{\mathrm{P} 2}=$ $31.75 \mathrm{eV}$.

1. Calculate the ionization rate as a function of phase using equation 4.1 .

2. Calculate the classical trajectory for an electron born at a given phase of the 
incident electric field using equation 4.5.

3. Find the "returns" to the nucleus by calculating the zeros of equation 4.5.

4. Calculate the kinetic energy of the electron at the time of return.

5. Weight the return by the ionization probability for an electron born at this phase.

6. Add this "probability of return" to the appropriate energy bin.

7. Repeat for the next phase of the incident laser field.

These "spectra" exhibit the features of a traditional harmonic spectrum. The -presence of an extended plateau is observed with an abrupt cut-off occurring at the predicted value. Even the relative magnitudes of the spectra for two intensities are correct with the signal dropping off approximately two orders of magnitude for a factor of two decrease in intensity. Harmonics which are close to or below the ionization potential of the atom cannot be studied with this technique since all bound state effects are ignored and they will be very important in this regime. However, this simple model appears to capture the essence of the process of high-order harmonic generation.

Now, if the problem is extended to two dimensions, for example if the laser field is no longer linearly polarized, a very interesting test of this model is possible. Since the primary influence on the trajectory of the electron is the strong laser field, adding a perpendicular field component should readily perturb this motion, acting to push the electron away from the nucleus more quickly than in the case of a linearly polarized field. Therefore, it would be expected that harmonic generation would cease very rapidly as the ellipticity of the laser field is increased. Here ellipticity is defined as the ratio of the $y$ (minor axis) to the $x$ (major axis) components of the laser field. In order to test this, the semiclassical model was extended to two dimensions with the influence of the atomic potential retained and several trajectories were examined as a function of the ellipticity of the field. 
Figure 4.6 shows a parametric plot of electron position, $y$ vs $x$, as well as a plot of its position as a function of phase, $r$ vs $\omega t$, for the case of an incident laser electric field magnitude equal to 0.5 that of the atomic field and an ellipticity of .1. The electron is born at a phase of 1.55 radians where, in the case of linearly polarized light, it experiences many returns. Since the influence of the atomic potential is now included, the electron can no longer be assumed to be born at $x=0$ or else it will simply remain bound to the nucleus. The laser field will only be able to drive electrons born some distance away from the origin since the strength of the potential is maximum at this point. For this calculation the position of the electron at birth is $1.1 a_{o}$ so the energy the electron has when it crosses the nucleus immediately after ionization is very small. Even with this relatively small value of ellipticity, the electron rapidly leaves the vicinity of the nucleus and never returns. Other trajectories for phases where the ionization probability is significant yield similar results. This lends weight to the argument that an experiment which allows for the variation of the degree of ellipticity of the incident laser could be used as a very sensitive test of this semiclassical interpretation.[budi193] Unfortunately, calculating the spectrum in the case of elliptical polarization is a very cumbersome calculation and beyond the scope of this work.

A second aspect of the process is accessible experimentally. This picture relies on the atom ionizing via tunneling where the electron very rapidly tunnels through the potential barrier and leaves the vicinity of the nucleus. By correct choice of the incident laser intensity and target gas, experiments can be conducted in the regime where multiphoton ionization is the dominant mechanism.[perry88] Here, the charge density remains very localized around the nucleus and the electron sequentially absorbs photons until its energy exceeds the ionization potential of the atom. These two regimes are defined by use of the Keldysh tunneling parameter,

$$
\gamma=\left(\frac{\mathrm{I}_{\mathrm{P}}}{2 \mathrm{U}_{\mathrm{P}}}\right)^{1 / 2}
$$

where for $\gamma<1$ tunneling will dominate and for $\gamma>1$ multiphoton ionization is dominant.[keldy65] Table 4.1 lists values of $\gamma$ for a variety of incident wavelengths 
(a)

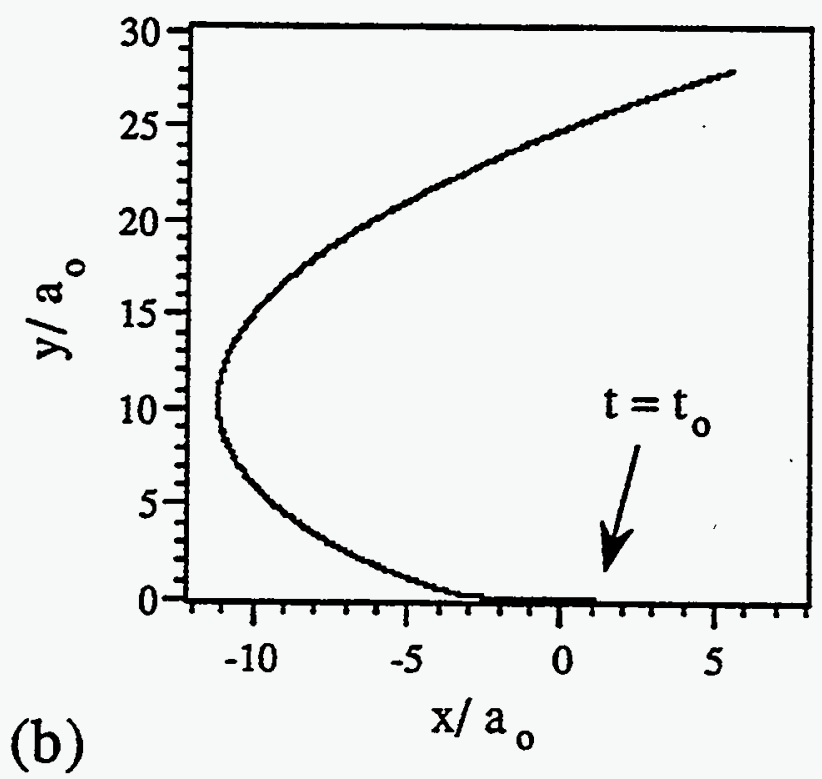

(b)

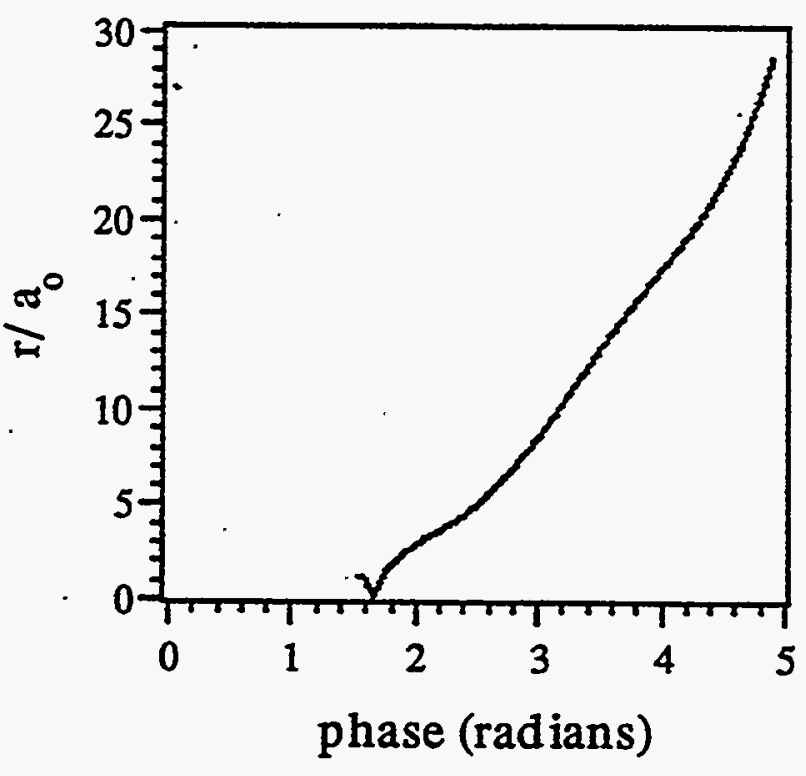

Figure 4.6: An example of a trajectory generated for the case where the polarization of the incident laser has an ellipticity of .1. (a) Plot of $y$ vs $x$ and (b) plot of $r$ vs $t$. where the influence of the atomic potential has been included. 


\begin{tabular}{|c|c|c|c|c|c|}
\hline gas & $I_{p}$ & $\lambda$ & intensity & $\mathrm{U}_{\mathrm{P}}$ & $\gamma$ \\
\hline \multirow[t]{2}{*}{ neon } & \multirow[t]{2}{*}{$21.6 \mathrm{eV}$} & \multirow[t]{2}{*}{$825 \mathrm{~nm}$} & $5 \times 10^{14} \mathrm{~W} / \mathrm{cm}^{2}$ & $31.8 \mathrm{eV}$ & 0.58 \\
\hline & & & $1 \times 10^{15} \mathrm{~W} / \mathrm{cm}^{2}$ & $63.5 \mathrm{eV}$ & 0.41 \\
\hline \multirow[t]{2}{*}{ argon } & \multirow[t]{2}{*}{$15.8 \mathrm{eV}$} & \multirow[t]{2}{*}{$825 \mathrm{~nm}$} & $5 \times 10^{14} \cdot \mathrm{W} / \mathrm{cm}^{2}$ & $31.8 \mathrm{eV}$ & 0.50 \\
\hline & & & $1 \times 10^{15} \mathrm{~W} / \mathrm{cm}^{2}$ & $63.5 \mathrm{eV}$ & 0.35 \\
\hline \multirow[t]{4}{*}{ xenon } & \multirow[t]{4}{*}{$12.1 \mathrm{eV}$} & \multirow[t]{2}{*}{$600 \mathrm{~nm}$} & $2 \times 10^{13} \mathrm{~W} / \mathrm{cm}^{2}$ & $0.67 \mathrm{eV}$ & 3.00 \\
\hline & & & $5 \times 10^{13} \mathrm{~W} / \mathrm{cm}^{2}$ & $1.68 \mathrm{eV}$ & 1.90 \\
\hline & & \multirow[t]{2}{*}{$825 \mathrm{~nm}$} & $5 \times 10^{13} \mathrm{~W} / \mathrm{cm}^{2}$ & $3.18 \mathrm{eV}$ & 2.18 \\
\hline & & & $1 \times 10^{14} \mathrm{~W} / \mathrm{cm}^{2}$ & $6.35 \mathrm{eV}$ & 0.98 \\
\hline
\end{tabular}

Table 4.1: Values of the Keldysh tunneling parameter for various experimental conditions.

and target gases. In either case harmonic generation is forbidden with a circularly polarized laser driver due to dipole selection rules.

\section{4:2 The Influence of Ellipticity}

In order to test the validity of the semiclassical model of high-order harmonic generation, an experiment was undertaken to examine the effect of ellipticity of the incident laser field. Several issues had to be resolved in order for this experiment to provide a meaningful test of the semiclassical model. Most important was the fact that the efficiency of the diffraction grating in the spectrometer is a function of polarization, having a maximum for vertically polarized light (light polarized parallel to the grooves). This was investigated by recording the magnitude of the signal for a given harmonic for both horizontal and vertical polarizations of the incident radiation and comparing these two values. This was repeated for several harmonics and the discrepancy was never greater than a factor of two, well within the error bar for the measurement of the number of harmonic photons produced. 
Second, the polarization of each laser utilized was checked using a thin film polarizer to ensure that pure linear polarization was present initially. The polarization of the incident laser was varied from linear to circular continuously by passing the beam through a broad-band (zero-order) quarter waveplate for the appropriate wavelength $(600 \mathrm{~nm}$ for the dye laser and $825 \mathrm{~nm}$ for the Cr:LiSAF laser). By utilizing an electron multiplier to detect the harmonic signal more than four orders of magnitude in dynamic range were achievable. In order to minimize phase matching effects a focal geometry was utilized which ensured that the confocal parameter was much greater than the length of the gas medium. These values were checked by recording the harmonic intensity as a function of the position of the focus within the jet. The focal spot was moved by translating the focusing lens along the axis of propagation and keeping the pulsed gas jet fixed. The position of the focus in the gas jet along the axis perpendicular to the direction of propagation was optimized by moving the jet transversely and again recording the harmonic signal. The final alignment of the lens was fixed at the center of the confocal parameter and the jet was set for maximum signal corresponding to the center of the exit aperture for the gas.

The first experiment utilized xenon as the target medium and the picosecond dye laser operating at $600 \mathrm{~nm}$ as the driver. A weak focusing geometry, $f^{\#}=30$, was utilized for these experiments corresponding to a $1 \mathrm{~cm}$ diameter beam being focused by a $30 \mathrm{~cm}$ focal length lens. The dye laser can be focused to 1.5 times the diffraction limit yielding intensities $\leq 10^{14} \mathrm{~W} / \mathrm{cm}^{2}$. Although harmonics were observed in argon, krypton, and xenon, the low conversion efficiencies made xenon the only medium utilized for these experiments as the goal was to study the signal over many orders of magnitude as it decreased. Figure 4.7 shows the ninth, eleventh, and thirteenth harmonics in xenon as a function of ellipticity. Each point corresponds to an average of 30 shots with a strict ( $\pm 15 \%)$ selection in energy. Here the intensity is $\sim 6 \times 10^{13} \mathrm{~W} / \mathrm{cm}^{2}$, yielding a $\gamma \sim 1.7$, in the multiphoton regime. Each harmonic has been normalized to a peak value of one to aid in comparison. The number of photons produced for each harmonic for all of the data presented herein is summarized 


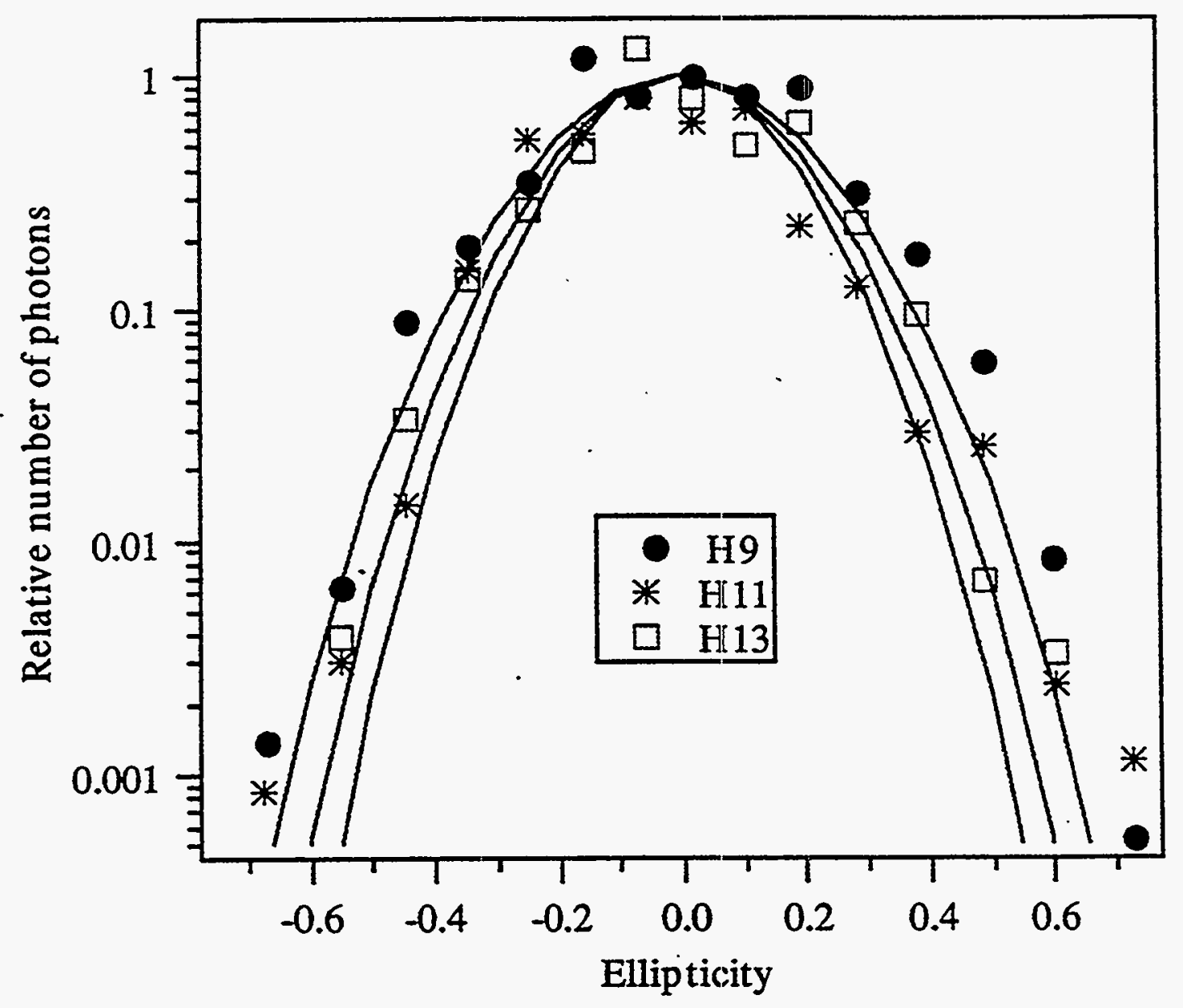

Figure 4.7: Harmonics in xenon as a function of ellipticity. The curves represent the LOPT predictions for the decrease in harmonic intensity as the ellipticity of the incident beam is increased with decreasing profile width corresponding to increasing harmonic order. 


\begin{tabular}{|c|c|c|c|c|c|c|c|}
\hline gas and $\lambda$ & order & intensity & $n$ & gas and $\lambda$ & order & intensity & $n$ \\
\hline \multirow{3}{*}{$\begin{array}{l}\text { xenon } \\
600 \mathrm{~nm}\end{array}$} & 7 & $6 \times 10^{13}$ & $4 \times 10^{8}$ & \multirow{8}{*}{$\begin{array}{l}\text { neon } \\
825 \mathrm{~nm}\end{array}$} & 15 & $1 \times 10^{15}$ & $3 \times 10^{6}$ \\
\hline & 9 & & $2 \times 10^{7}$ & & 17 & & $3.7 \times 10^{6}$ \\
\hline & 11 & & $4 \times 10^{5}$ & & 19 & & $3 \times 10^{6}$ \\
\hline \multirow{5}{*}{$\begin{array}{l}\text { argon } \\
825 \mathrm{~nm}\end{array}$} & 15 & $3.5 \times 10^{14}$ & $2 \times 10^{8}$ & & 21 & & $3 \times 10^{6}$ \\
\hline & 17 & & $3 \times 10^{8}$ & & $\overline{33}$ & & $6 \times 10^{6}$ \\
\hline & $\overline{19}$ & & $4 \times 10^{8}$ & & 43 & & $4 \times 10^{6}$ \\
\hline & 21 & & $5 \times 10^{8}$ & & 53 & & $2.0 \times 10^{6}$ \\
\hline & 29 & & $1.3 \times 10^{8}$ & & 63 & & $1.5 \times 10^{6}$ \\
\hline \multirow{2}{*}{$\begin{array}{l}\text { xenon } \\
825 \mathrm{~nm}\end{array}$} & 13 & $1.2 \times 10^{14}$ & $3.5 \times 10^{9}$ & \multirow{2}{*}{$\begin{array}{l}\text { xenon } \\
825 \mathrm{~nm}\end{array}$} & 17 & $1.2 \times 10^{14}$ & $5 \times 10^{8}$ \\
\hline & 15 & & $2.2 \times 10^{9}$ & & 19 & & $3.5 \times 10^{7}$ \\
\hline
\end{tabular}

Table 4.2: Maximum number of photons produced for each harmonic order and generating medium presented herein. Intensity is presented in units of $W / \mathrm{cm}^{2}$. For the $600 \mathrm{~nm}$ results, $f^{\#}=30$, and for the $825 \mathrm{~nm}$ results, $f^{\#}=24$.

in Table 4.2. These data were compared to lowest-order perturbation theory (LOPT). The form of the nonlinear polarization of the $q$ th harmonic produced by an elliptically polarized pump source has been shown to be [manak80]

$$
\mathrm{P}^{(q)}(q \omega)=\vec{e} \mathrm{~F}\left(\frac{\mathrm{DF}^{2}}{4}\right)^{(q-1) / 2} \chi^{(q)}(-q \omega)
$$

where $\chi^{(q)}(-q \omega)$ is the nonlinear susceptibility, D is the degree of linear polarization,

$$
\mathrm{D}=\frac{1-\epsilon^{2}}{1+\epsilon^{2}}
$$

and $F$ is the pump field defined by

$$
\mathbf{F}(\mathrm{r}, t)=\mathrm{F} \Re e\left[\vec{e} e^{-i(\omega t-k z)}\right]
$$

where

$$
\vec{e}=\frac{\hat{e}_{x}-i \epsilon \hat{e}_{y}}{\left(1+\epsilon^{2}\right)^{1 / 2}}
$$

defines the polarization of the incident laser field. The polarization can vary from linear, $\epsilon=0$, to circular, $\epsilon=1$. Since the intensity of the $q$ th harmonic is proportional 
to $|\mathrm{P}(q \omega)|^{2}$, the intensity of the $q$ th harmonic will vary as

$$
I_{q} \propto\left(\frac{1-\epsilon^{2}}{1+\epsilon^{2}}\right)^{(q-1)}
$$

where $\epsilon$ is the ellipticity as defined above. [liang:94] The theoretical curves are decreasing in width for increasing harmonic order. A relatively weak dependence on ellipticity is observed in the data in good agreement with the predictions of LOPT. To obtain a one order of magnitude decrease in the harmonic signal an ellipticity of nearly .4 is required.

The remaining experiments were conducted with the Cr:LiSAF laser system operating at $825 \mathrm{~nm}$. A comparable focal geometry, $f^{\#}=24$, is constructed by using a $1 \mathrm{~m}$ focal length lens to focus the much larger LiSAF beam $(\mathrm{d}=40 \mathrm{~mm})$. Now focused intensities of $\geq 10^{15} \mathrm{~W} / \mathrm{cm}^{2}$ are readily achievable due to the much higher energies and shorter pulse widths which the LiSAF delivers. The first gas used as a generating medium was neon with an incident laser intensity $\sim 10^{15} \mathrm{~W} / \mathrm{cm}^{2}$. In this high intensity regime the laser field may no longer be treated as a small perturbation on the atomic potential. Particularly harmonics well above the ionization threshold of neon $(21.6 \mathrm{eV})$ are expected to be well described by the two-step semiclassical model. Tunneling is the dominant ionization mechanism here with $\gamma=0.41$. Figure 4.8 presents harmonics obtained in neon.

As the harmonic order is increased, the distributions become narrower with a pronounced transition occuring between the 17th and 19th harmonics. Above the 19th order, the dependence of the harmonic intensity on ellipticity is quite strong and quite similar for all orders. The signal level has begun to drop off with less than 0.1 ellipticity and has decreased by more than one order of magnitude for an ellipticity of 0.2 . The lower orders require 1.5 times the ellipticity to see the same decrease in signal. It is also interesting to note that the dependence here is in general not as steep as that predicted by LOPT for high orders.

The next gas to be used as a generating medium was argon with the Cr:LiSAF laser again serving as the driver. Here the ionization potential is lower $(15.6 \mathrm{eV})$ 


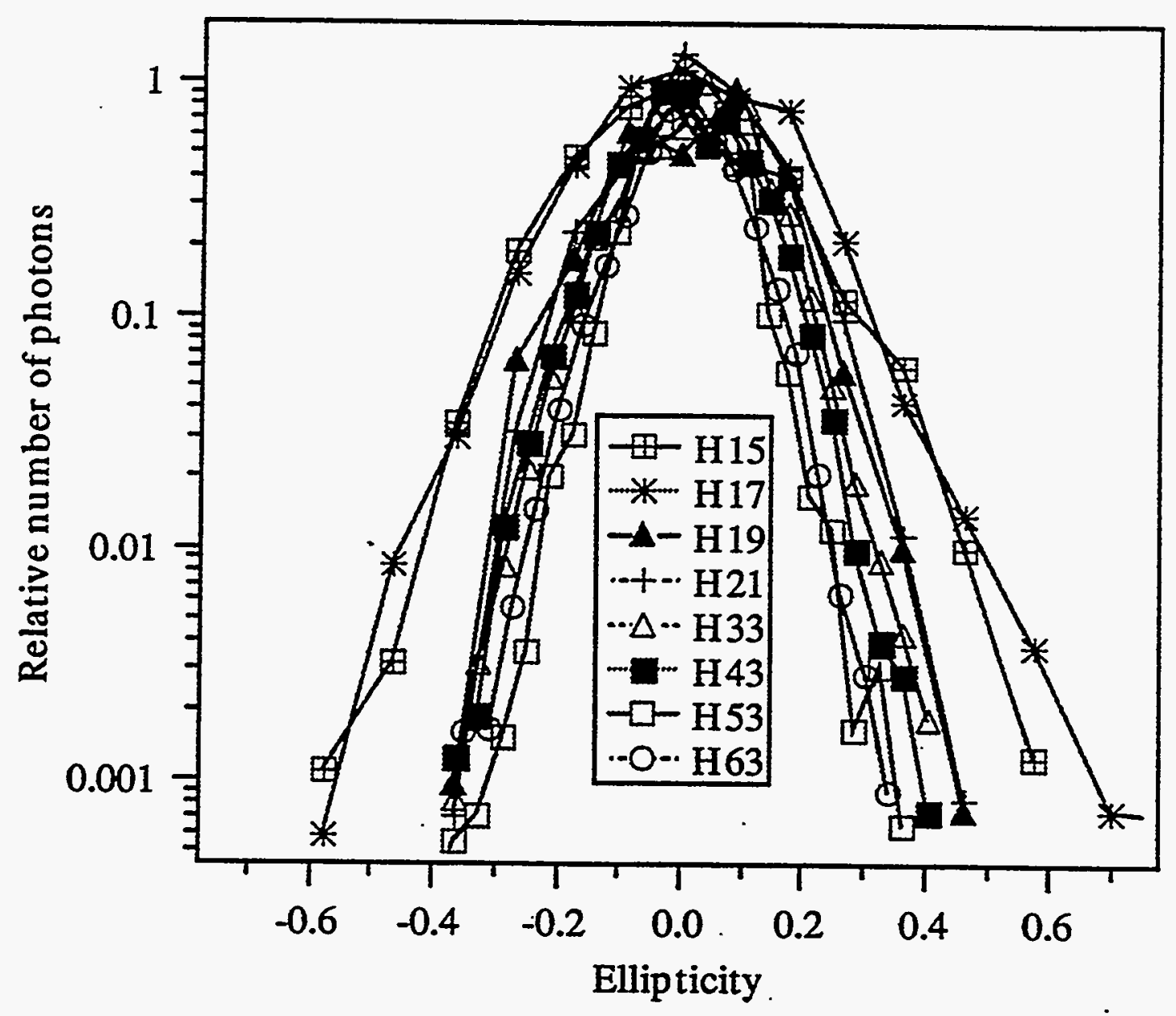

Figure 4.8: Harmonics in neon as a function of ellipticity. The incident laser wavelength is $825 \mathrm{~nm}$ and the intensity is $\sim 1 \times 10^{15} \mathrm{~W} / \mathrm{cm}^{2}$. 
and fewer harmonics are observed. The fifteenth, seventeenth, nineteenth, twentyfirst, and twenty-ninth harmonics are presented in Figure 4.9. Here $\gamma \sim 0.59$. The

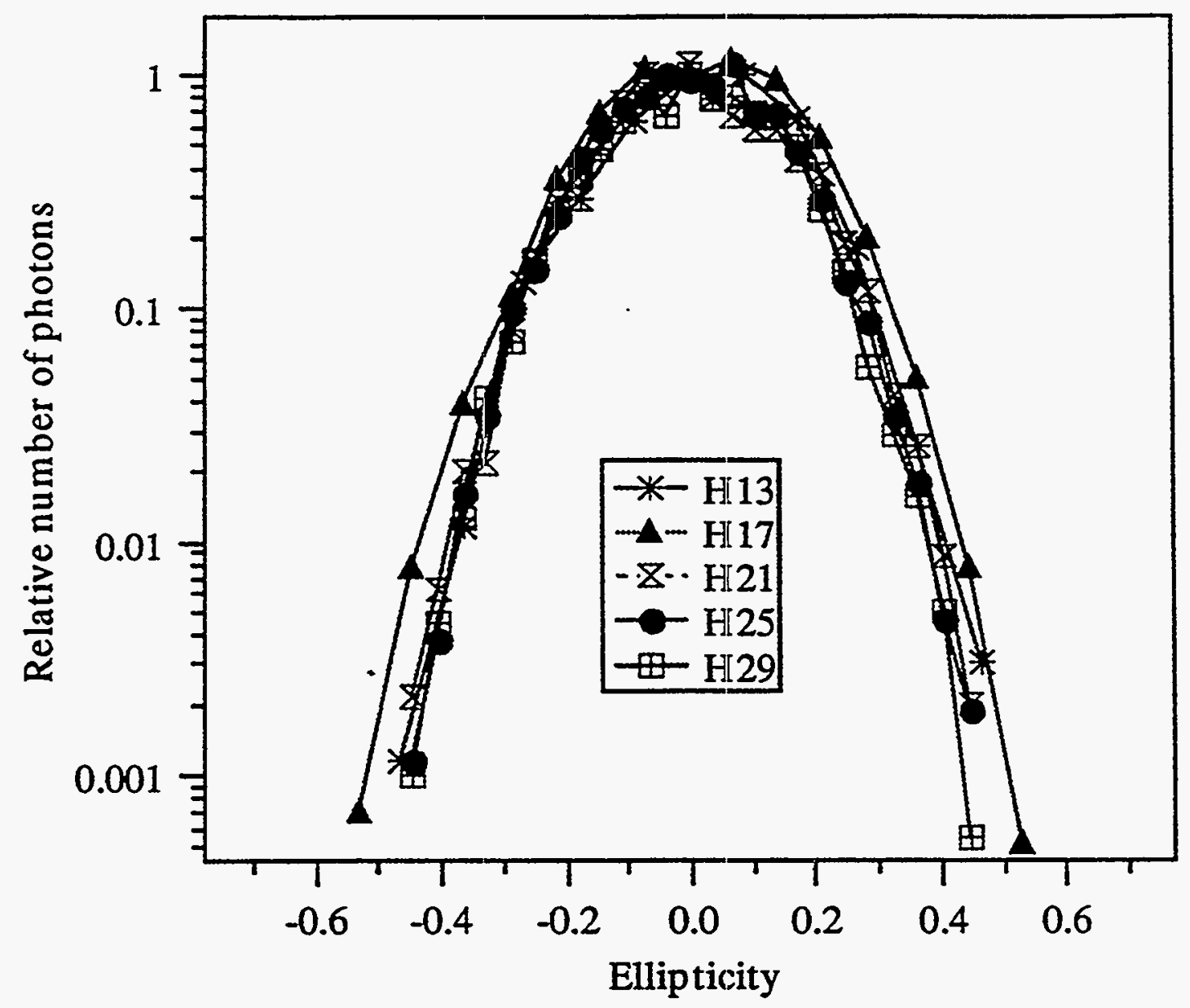

Figure 4.9: Harmonics in argon as a function of ellipticity. The intensity is $3.5 \times$ $10^{14} \mathrm{~W} / \mathrm{cm}^{2}$ and $\lambda=825 \mathrm{~nm}$.

dependence observed here falls between those of the highest and lowest orders in neon.

Figure 4.10 shows harmonics produced in xenon with the LiSAF laser system. The ionization potential is again lower $(12.1 \mathrm{eV})$ so even fewer harmonics are observed due to the ease of ionization of the medium. The value of the tunneling parameter is $\gamma \sim 0.89$. The decrease in harmonic intensity due to increasing ellipticity is only slightly sharper than when the dye laser was utilized. In contrast to the dye laser 


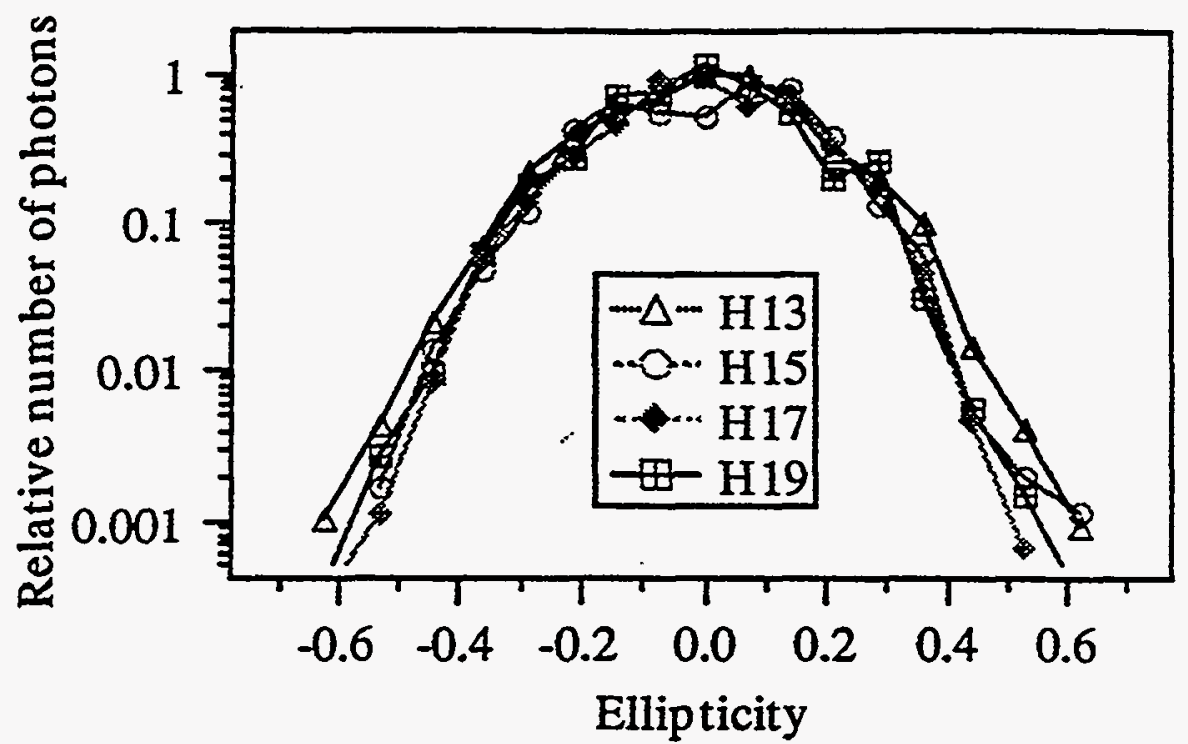

Figure 4.10: Harmonics in xenon as a function of ellipticity. The intensity is $1.2 \times$ $10^{14} \mathrm{~W} / \mathrm{cm}^{2}$.

results, these data do not agree with the predictions of LOPT.

The most interesting feature observed in these data is the abrupt transition between the seventeenth and nineteenth harmonics observed in neon with the Cr:LiSAF laser. Figure 4.11 shows the harmonic spectrum obtained in neon as presented earlier where the nineteenth harmonic in neon appears at the beginning of the plateau. Figure 4.12 shows the seventeenth, nineteenth, and twenty-first harmonics respectively for each of the gases studied with the LiSAF laser for similar intensities. The behavior of the seventeenth and twenty-first harmonics is very similar in each case with a slight steepening of the dependence on ellipticity for each increase of the ionization potential. However, there is a clear shift in neon at the nineteenth harmonic from the regime of weak dependence to that of strong dependence. In general LOPT predicts a steeper dependence for high orders than is observed experimentally. It is interesting.to note that the dependence on ellipticity is fairly independent of harmonic order with the only shift being from the "weak dependence" or "low order" regime to the "strong dependence" or "high order" regime. 


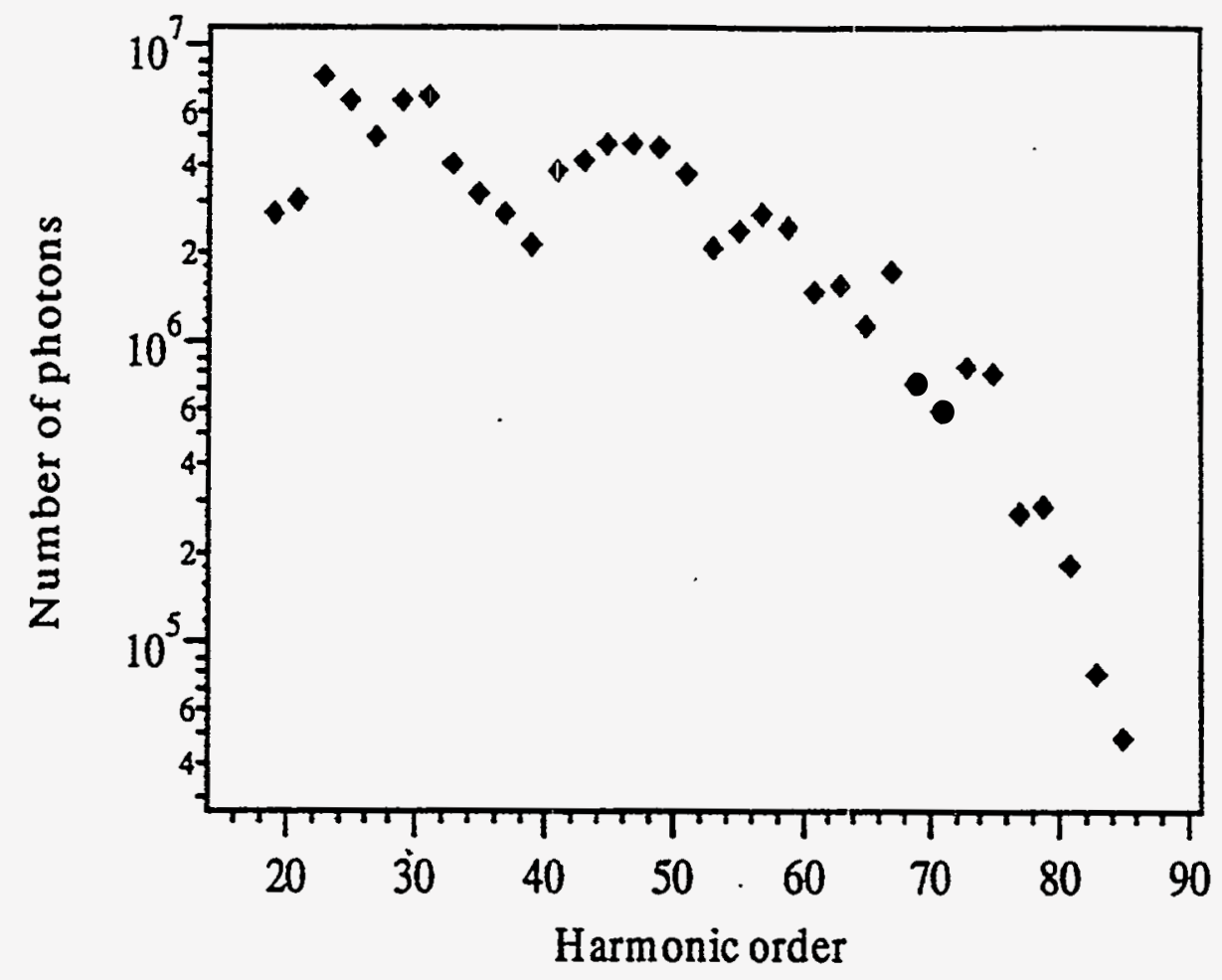

Figure 4.11: Harmonic spectrum obtained in neon at an intensity of $3.3 \times 10^{14} \mathrm{~W} / \mathrm{cm}^{2}$ with the Cr:LiSAF laser operating at $825 \mathrm{~nm}$.

\subsection{Simple Quantum Theory of Harmonic Gen- eration}

Since the two step semiclassical model becomes unwieldy when extended to two dimensions, an alternate theory was applied to examine the data. This approach, developed by Lewenstein, et al. [lewen94], is fully quantum mechanical thereby easily incorporating the nonclassical elements of the process such as quantum diffusion of the wave packet and interferences. Its range of validity is determined by several assumptions. First, the contributions of all bound states excluding the ground state are 
(a)

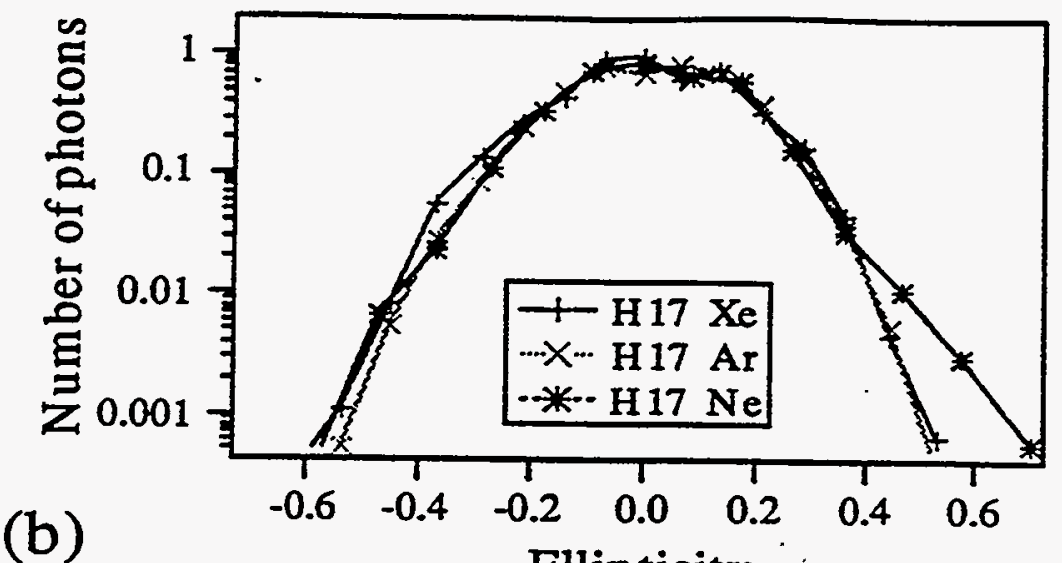

(b)

Ellip ticity

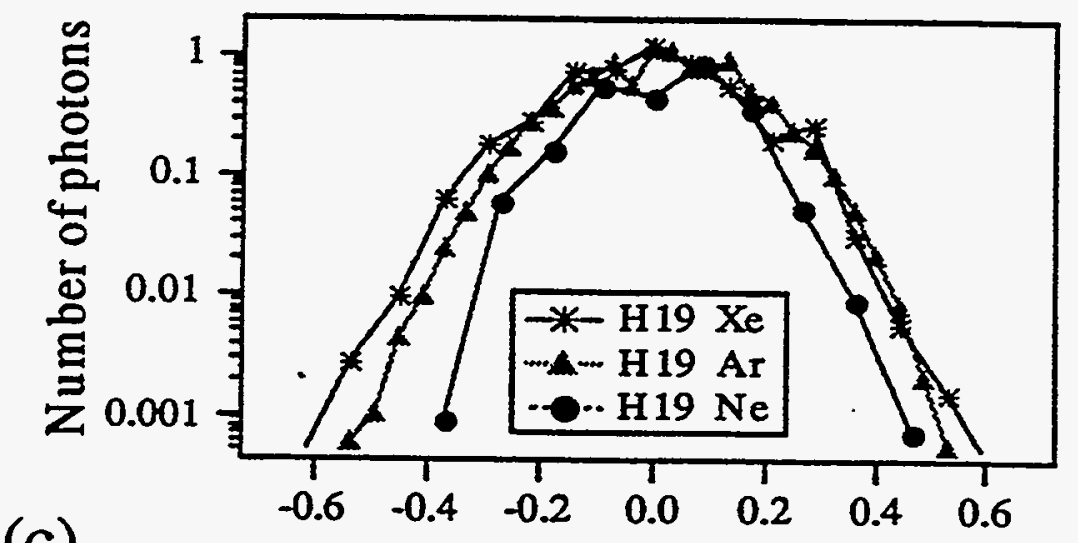

(c)

Ellip ticity

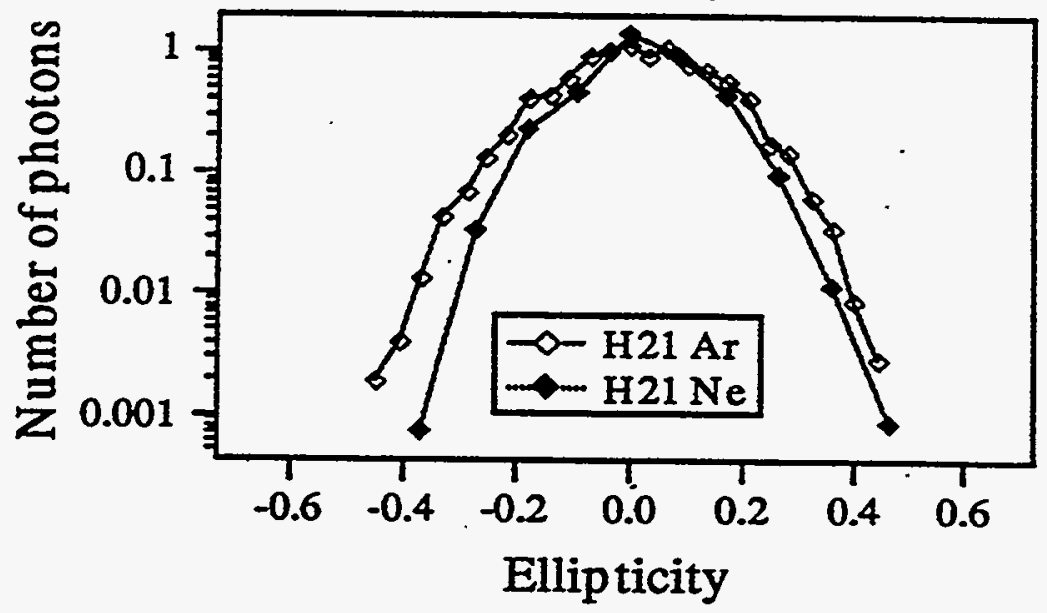

Figure 4.12: The 17th, 19th, and 21st harmonics as a function of ellipticity for neon, argon, and xenon. The 21st harmonic was not observed in xenon. 
neglected which will be true if there are no intermediate resonances and when tunneling ionization is dominant, $\gamma<1$. The depletion of the ground state is neglected which assumes that the intensity is less than the saturation intensity. And finally, the electron is treated as a free particle moving in the laser field and the influence of the atomic potential is neglected. As discussed before, this assumption will hold if the driving electric field is very strong so that the electron will have a large kinetic energy as it passes the nucleus. Therefore, only harmonics above the ionization potential will be treated.

\subsubsection{Theoretical Approach}

The atom is considered to be under the influence of a laser field of arbitrary polarization and the Schrödinger equation, in the length gauge, takes the form $(\hbar=\mathrm{m}=1)$ [lewen94]

$$
i \frac{\partial}{\partial t}\left|\Psi(\vec{x}, t)>=\left[\frac{-1}{2} \nabla^{2}+V(\vec{x})-E_{\text {laser }}(t) \vec{x}\right]\right| \Psi(\vec{x}, t)>
$$

and the system is initially in the ground state $|0\rangle$. If the influence of the atomic potential is neglected and the laser field has the form $E_{\text {laser }}=E \cos (t)$ this becomes

$$
i \frac{\partial}{\partial t}|\Psi(\vec{x}, t)\rangle=\left[\frac{-1}{2} \nabla^{2}-E \cos (t) \vec{x}\right] \mid \Psi(\vec{x}, t)>.
$$

The time-dependent wave function can then be expanded as

$$
\mid \Psi(t)>=e^{i \mathrm{I}_{\mathrm{P}} t}\left[a(t)\left|0>+\int d^{3} \vec{v} b(\vec{v}, t)\right| \vec{v}>\right]
$$

where $a(t) \simeq 1$ is the ground state amplitude and $b(\vec{v}, t)$ are the amplitudes of the corresponding continuum states with momenta $\vec{v}$ and oscillations of the ground state with frequency $\mathrm{I}_{\mathrm{P}}$ have been factored out. The Schrödinger equation for $b(\vec{v}, t)$ is then given by

$$
\frac{\partial b(\vec{v}, t)}{\partial t}=-i\left(\frac{v^{2}}{2}+I_{P}\right) b(\vec{v}, t)-\mathrm{E} \cos (t) \frac{\partial b(\vec{v}, t)}{\partial v_{x}}+i \mathrm{E} \cos (t) d_{x}(\vec{v})
$$

where $\mathrm{d}(\vec{v})=\langle\vec{v}|\vec{x}| 0\rangle$ is the atomic dipole matrix element for the bound-free transition and $d_{x}(\vec{v})$ is the component parallel to the polarization axis. Equation 4.15 
may be solved for $b(\vec{v}, t)$ exactly yielding,

$$
b(\vec{v}, t)=i \int_{0}^{t} d t^{\prime} \mathrm{E}_{\text {laser }}(t) d_{x}\left[\vec{v}+\overrightarrow{\mathrm{A}}(t)-\overrightarrow{\mathrm{A}}\left(t^{\prime}\right)\right] e^{-i \int_{t}^{t^{\prime}} d t^{\prime \prime}\left[\left(\vec{v}+\overrightarrow{\mathrm{A}}(t)-\overrightarrow{\mathrm{A}}\left(t^{\prime \prime}\right)\right)^{2} / 2+\mathrm{I}_{\mathrm{P}}\right]}
$$

where $\vec{A}(t)$ is the vector potential of the laser field. The harmonic spectrum is obtained by calculating the time-dependent dipole moment where

$$
\begin{aligned}
x(t) & =\langle\Psi(t)|x| \Psi(t)\rangle \\
& =\int d^{3} \vec{v} d_{x}^{*}(\vec{v}) b(\vec{v}, t)+c . c . \\
& =\int d^{3} \vec{v} d_{x}^{*}(\vec{v}) i \int_{0}^{t} d t^{\prime} \mathrm{E}_{\text {laser }}(t) d_{x}\left[\vec{v}+\vec{A}(t)-\overrightarrow{\mathrm{A}}\left(t^{\prime}\right)\right] e^{-i \int_{t}^{t^{\prime}} d t^{\prime \prime}\left[\left(\vec{v}+\overrightarrow{\mathrm{A}}(t)-\overrightarrow{\mathrm{A}}\left(t^{\prime \prime}\right)\right)^{2} / 2+\mathrm{I}_{\mathrm{P}}\right]}+\text { c.c. }
\end{aligned}
$$

By introducing a canonical momentum $\vec{p}=\vec{v}+\vec{A}(t)$.this can be expressed as

$$
\begin{aligned}
x & =\int d^{3} \vec{v} d_{x}^{*}(\vec{v}) i \int_{0}^{t} d t^{\prime} \mathrm{E}_{\text {laser }}(t) d_{x}\left[\vec{p}-\vec{A}\left(t^{\prime}\right)\right] e^{-i \int_{t}^{t^{\prime}} d t^{\prime \prime}\left[\left(\vec{p}-\overrightarrow{\mathrm{A}}\left(t^{\prime \prime}\right)\right)^{2} / 2+\mathrm{I}_{\mathrm{P}}\right]}+c . c . \\
& =i \int_{0}^{t} d t^{\prime} \int d^{3} \vec{p} \mathrm{E}_{\text {laser }}(t) d_{x}\left(\vec{p}-\overrightarrow{\mathrm{A}}\left(t^{\prime}\right)\right) d_{x}^{*}(\vec{p}-\overrightarrow{\mathrm{A}}(t)) e^{-i S\left(\vec{p}, t t^{\prime}\right)}+c . c .
\end{aligned}
$$

where

$$
S\left(\vec{p}, t, t^{\prime}\right)=\int_{t^{\prime}}^{t} d t^{\prime \prime}\left[\frac{\left(\vec{p}-\vec{A}\left(t^{\prime \prime}\right)\right)^{2}}{2}+I_{P}\right]
$$

is the quasiclassical action.

Equation 4.18 can be interpreted as a sum of probability amplitudes for the electron making a transition to the continuum at time $t^{\prime}$ and then recombining at time $t$, respectively. It is also interesting to note that the primary contribution to equation 4.18 comes from the stationary points of the classical action,

$$
\nabla_{\vec{p}} S\left(\vec{p}, t, t^{\prime}\right)=0=\vec{x}(t)-\vec{x}\left(t^{\prime}\right)
$$

which is simply the change in position of the electron between times $t$ and $t^{\prime}$. If the electron is born at $t^{\prime}$ and returns to this position again at time $t$, then $\vec{x}(t)$ must be close to the origin since only when the electron is near the nucleus can it recombine. Thus the dominant contribution to harmonic generation will be electrons which are born via tunneling away from the nucleus but then "return" while oscillating in the laser field, one of the primary assumptions of the two step semiclassical model. 
Lewenstein, et al.,[lewen94] also point out that the stationary point of the classical action corresponds to zero initial velocity for the electron, a second assumption of the two-step model.

The Fourier components of $x(t)$ may be calcalated by using a saddle point method to evaluate the integral over $\vec{p}$ in 4.18 yielding

$$
x_{2 K+1}=i \sum_{M}^{\infty} \int_{0}^{\infty} d \tau\left(\frac{\pi}{\epsilon+i \tau / 2}\right)^{3 / 2} e^{-i D_{0}(\tau)} b_{K-M}(\tau) J_{M}\left(\mathrm{U}_{\mathrm{P}} \mathrm{A}(\tau)\right)(i)^{M} e^{i M \tau}
$$

where $D_{0}(\tau)=\left(\mathrm{U}_{\mathrm{P}}+\mathrm{I}_{\mathrm{P}}\right) \tau-2 \mathrm{U}_{\mathrm{P}}(1-\cos (\tau)) / \tau$. The cut-off law is shown to have the form

$$
(2 K+1)_{\max }=3.17 \mathrm{U}_{\mathrm{P}}+\mathrm{I}_{\mathrm{P}} F\left(\mathrm{I}_{\mathrm{P}} / \mathrm{U}_{\mathrm{P}}\right)
$$

where $F\left(I_{P} / U_{P}\right)$ is slowly decreasing as its argument increases and in the range $\mathrm{I}_{P} / \mathrm{U}_{\mathrm{P}} \sim 1-4, F\left(\mathrm{I}_{\mathrm{P}} / \mathrm{U}_{\mathrm{P}}\right) \sim 1.3$ which is in good agreement with the phenomenological law. The integration over $\tau$ will be performed numerically utilizing a code [lewen94b] wherein the ground state $s$-wave function takes the form

$$
\Psi(\vec{x})=\left(\frac{\alpha^{3 / 4}}{\pi^{1 / 2}}\right) e^{-\alpha^{1 / 2}|\vec{x}|},
$$

where $\alpha=2 \mathrm{I}_{\mathrm{P}}$.

\subsubsection{Model Calculations}

The initial conditions for doing model calculations with this code are the intensity and wavelength of the driving laser (and thus the ponderomotive potential), the target species, and the order of the harmonic to be studied. As previously mentioned, this calculation is optimal for harmonics which are above the ionization potential of the gas being studied and reside in the plateau. The experimental data was used as a starting point and those harmonics which fit the above criterion were modeled using the actual experimental conditions as the initial conditions.

Since neon provides the most extended plateau of the gases studied, it provides a good starting point for testing the model over a broad range in wavelength. Figure 
4.13 shows the results of the model calculation for the twenty-first and thirty-third harmonics and Figure 4.14 the forty-third and fifty-third harmonics produced in neon with the LiSAF laser. The calculated signal level has been normalized to one. While the model calculations show oscillations of up to one order of magnitude, in each case the drop off in harmonic signal as ellipticity is increased is in very good agreement with the experimental data. The oscillations seem to worsen with increasing harmonic order. Since the effective coherence length of the process of harmonic generation is a function of the order of the process, $\sim(b / 2) \tan [\pi /(q-1)]$ where $b$ is the laser confocal parameter, higher order harmonics are effectively produced in the regime of tight focusing where phase matching effects will be very important.[Ihuil92] It is possible that if propagation were added to this calculation, some of this oscillatory behavior would be eradicated by phase matching effects and averaging over the spatiotemporal intensity distribution. Alternatively, the oscillations may arise numerically so that decreasing the time step should lead to diminishing error. The time step utilized here yielded the best results, however it was noted that the parameter of interest in these calculations, the width of the distributions, did not vary significantly as a function of the time step.

It is interesting to note that while perturbation theory predicts a steeper decrease than that observed experimentally, the quantum model produces essentially the same dependence on ellipticity as the experimental data. This model takes into account several aspects of the process that are purely quantum mechanical, in particular, the quantum diffusion of the wave packet. Even though the electron is being driven away from the nucleus by the addition of a small $y$ component of the laser. field, the wave packet is spreading as it moves through space. This increased spatial extent of the wave packet will alter the overlap between the electron and the ground state wave function of the atom, allowing for a finite dipole matrix element even when the electron, considered as a particle, is "far" from the nucleus.

Figure 4.15 shows the results of calculations for the nineteenth and twenty-first harmonics produced in argon by the LiSAF laser: Here the oscillations are smaller 

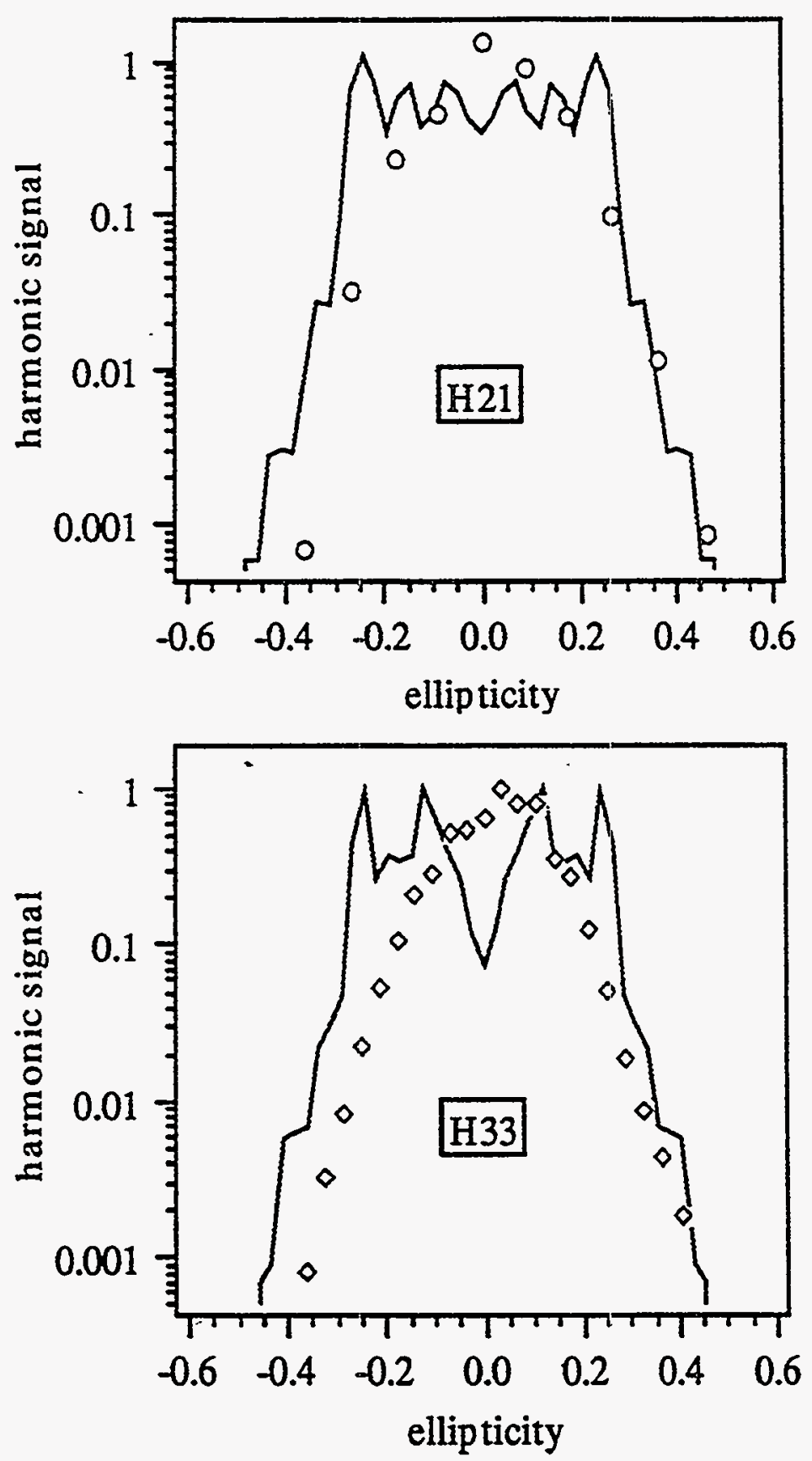

Figure 4.13: Harmonic signal as a function of ellipticity for the 21st and 33rd harmonics produced in neon. The open symbols represent the experimental data from the previous section and the solid line is the model calculation. 

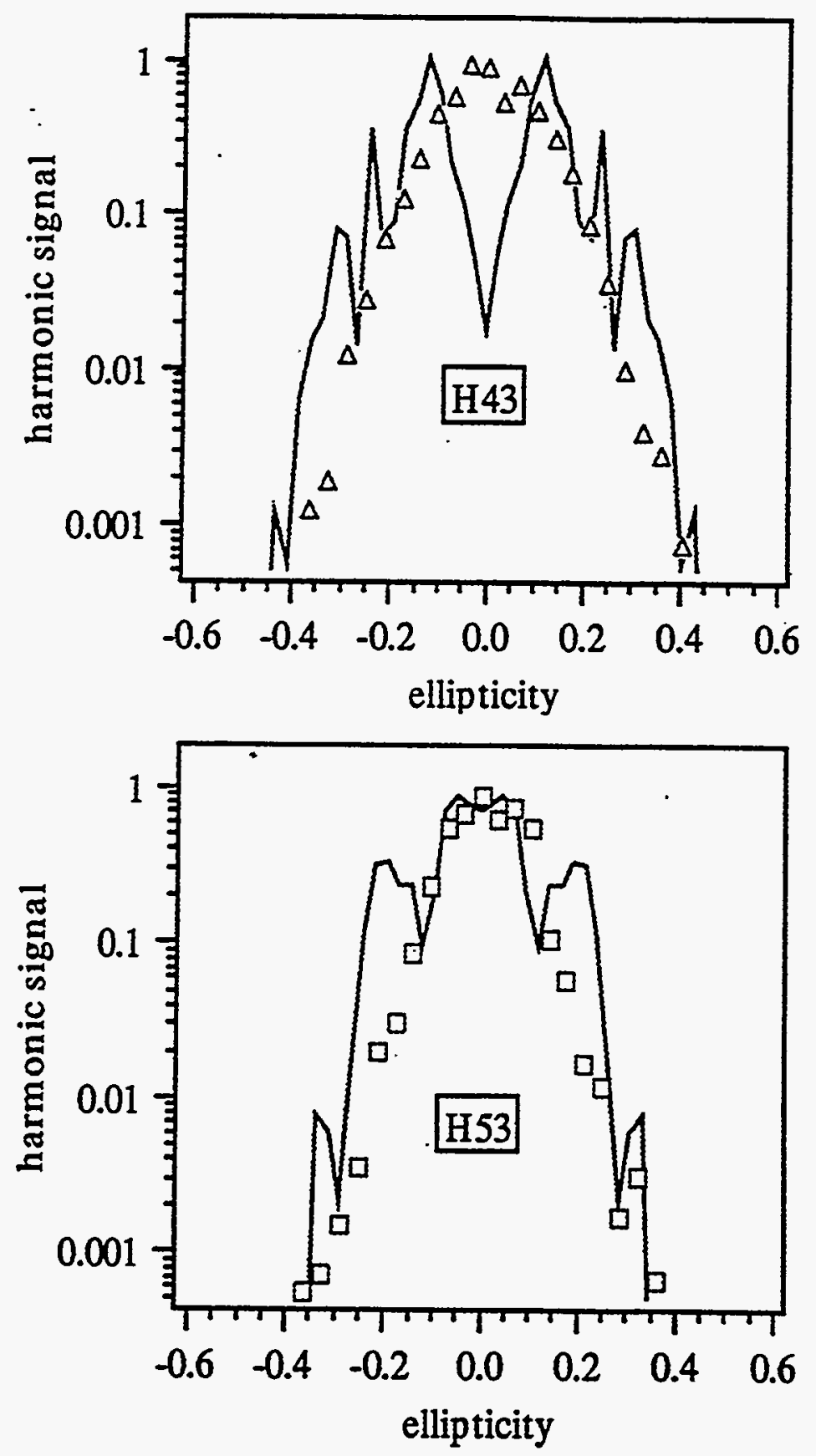

Figure 4.14: Harmonic signal as a function of ellipticity for the 43rd and 53rd harmonics produced in neon. The open symbols are experimental data and the solid line is the model calculation. 

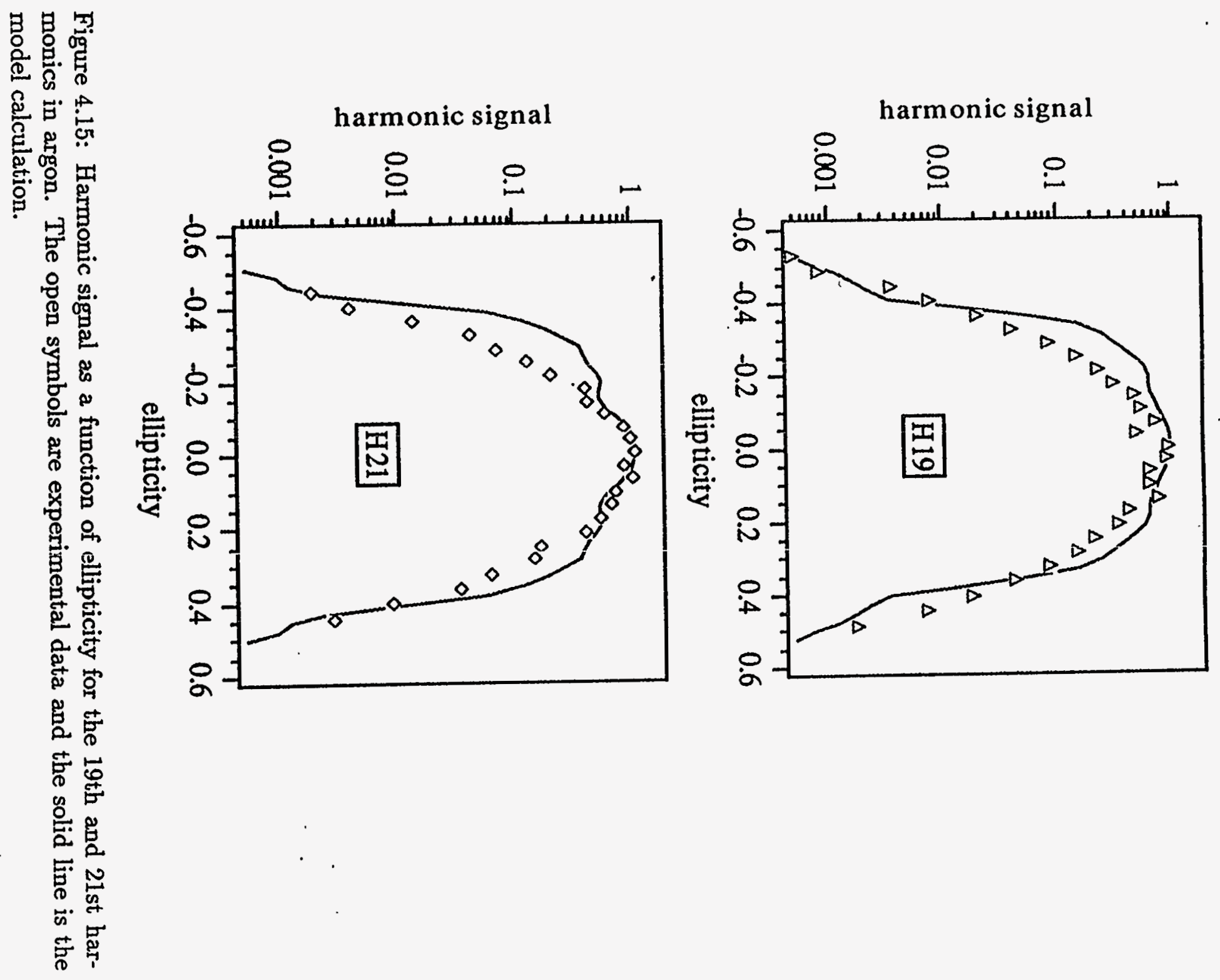
and the data are in very good agreement with the model predictions.

The final species studied was xenon. Figure 4.16 shows the seventeenth harmonic produced by the LiSAF laser. Because an extended plateau is not observed in xenon,

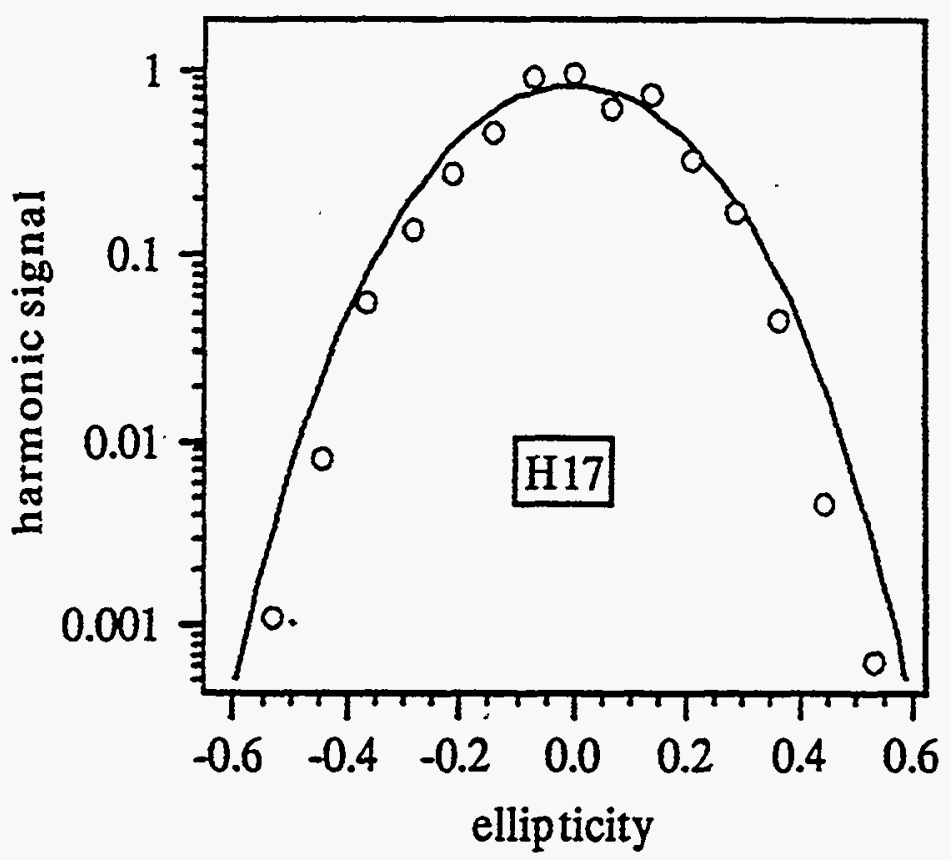

Figure 4.16: Harmonic signal as a function of ellipticity for the 17th harmonic in xenon. The open symbols are experimental data and the solid line is the model calculation.

this was the only harmonic modeled. Here the oscillations have completely vanished and the dependence of the harmonic signal on ellipticity is well matched by the model calculation.

Thus the quantum model described above provides good agreement with the experimental results obtained in the investigation of the influence of ellipticity on harmonic generation. Since this model recovers many of the conclusions of the two-step semiclassical model, the success of the quantum model tends to validate them both. 
The quantum model has the advantage of a smaller calculation and the direct inclusion of quantum mechanical effects which will be important in the two-dimensional case and would have to be added on to the classical calculation. 


\section{Chapter 5}

\section{The Harmonic "Source"}

\subsection{Background and Motivation}

Many areas of physics have long relied on spectroscopic techniques for investigating the characteristics of physical systems. Light sources providing radiation in the vacuum ultraviolet (VUV) and extreme ultraviolet (XUV) regions of the spectrum have allowed these techniques to be applied to an even larger class of problems. The construction of large synchrotron facilities has resulted in a renewed interest in VUV and XUV spectroscopy in studies of atomic and molecular states,[page88] molecular dynamics,[ng91] and surface states.[haigh93] Low order harmonics, typically third through ninth, have been utilized as sources of coherent VUV with both dye [miyaz89] and excimer [bokor83, egger80] lasers serving as the driver and sometimes exploiting intermediate two photon resonances. Since these experiments were performed in the regime where perturbation theory is valid, the rapidly decreasing conversion efficiency did not allow for their extension to higher order harmonics.

With the advent of high power lasers and the subsequent extension of harmonic generation to very high orders $(\geq 100 \mathrm{eV})$ interest in the applications of harmonic generation has been renewed. Optimization of the source for maximum conversion efficiency and best quality of the spatial profile of the harmonics was required as well as a system to deliver the harmonic radiation to the target chamber. 


\subsection{Characterization of the Source}

While a great deal had been learned about methods for optimizing the conversion efficiency of the source (optimal target density, laser power, phase matching conditions) little effort had been directed at examining the quality of the spatial profile of the harmonic radiation.[tisch94, peatr93] The spatial coherence of the harmonics is very important if they are to be utilized for holographic or lithographic purposes as well as any application where they will be focused. Therefore, a systematic study of the spatial coherence properties of high order harmonic radiation was undertaken.

In order to examine the spatial profile of the harmonics an imaging detection system was placed at the exit of the flat-field spectrometer. An imaging microchannel plate detector (Galileo VUV-25) was utilized to record the spatial and spectral profile of the harmonic beam. This consists of a CsI coated microchannel plate placed in . chevron configuration with a second uncoated plate. The electrons leaving the back plate are incident upon a phosphorus screen. The image on the phosphorus screen is then read by an 8-bit CCD camera and recorded with a PC-based data acquisition system. The detector is placed $13 \mathrm{~cm}$ beyond the focal plane of the spectrometer to prevent damage. The experimental apparatus is shown in Figure 5.1. Because the spectrometer is designed to produce a spectrum focused onto a flat field, several harmonics could easily be imaged at once. The vertical axis of images obtained in this manner shows the purely spatial characteristics of the harmonic. The horizontal axis is a convolution of the spectral characteristics with the spatial information since the harmonic is allowed to diverge past the focal plane of the spectrometer. In order to eliminate questions regarding the shot-to-shot fluctuations of the laser, an averaging routine was added to the data acquisition software. This allowed several laser shots to be taken within a given energy window (typically $\pm 10 \%$ ) and the images thus obtained were averaged. Given good enough energy discrimination, however, the images are very reproducible on a shot-to-shot basis and the averging served only to enhance the signal-to-noise ratio.

A contour plot of an image of the twenty-first, twenty-third, and twenty-fifth 


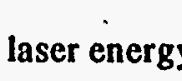

sample-and-hold amplifier and gated integrator

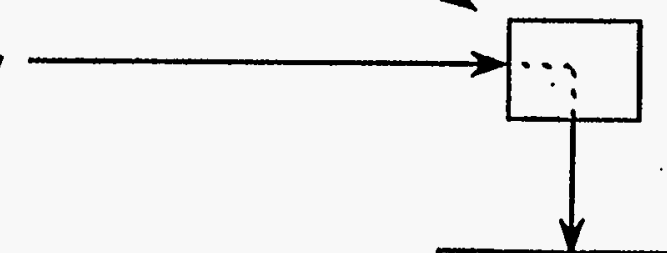

2-dimensional image of harmonic

pc-based data acquisition system

gold-coated variable groove density
plane diffraction grating

ccd microchannel camera plate detector
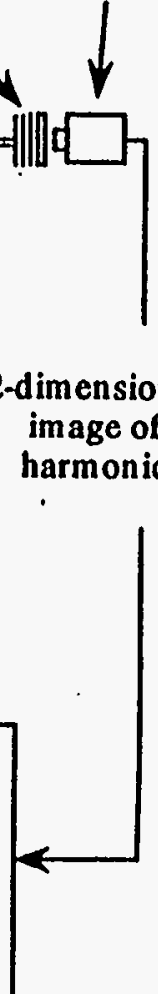
harmonics produced in argon with the LiSAF laser at an intensity of $1.2 \times 10^{14} \mathrm{~W} / \mathrm{cm}^{2}$ is shown in Figure 5.2. The ordinate contains information on the spatial distribution while the abscissa represents the spectral distribution. A vertical lineout integrated along the full spectral width of each harmonic was taken and is presented in Figure 5.3. The central contour for each harmonic represents the highest photon flux with each successive contour being a slightly lower signal level. As this detector has not been calibrated for absolute photon yield, the images presented depict signal level in relative units. The absolute harmonic yields for each of the rare gases are presented in Chapter 4. The peak signal has been normalized to one for all orders. The spatial profiles are symmetric and virtually without structure. A gaussian fit was applied to each of the profiles and shows good agreement with the experimental measurement. The divergence, defined as the full width of the profile at the $1 / e^{2}$ point, decreases from approximately $10 \mathrm{mrad}$ at the twenty-first harmonic to $\sim 6 \mathrm{mrad}$ at the twentyfifth.

The divergence of the LiSAF laser beam after focus was measured to be $55 \mathrm{mrad}$. [salie94] A simple estimate of the expected divergence can be made by assuming a gaussian distribution and calculating the far-field divergence angle expected. If we further assume that the intensity of the $q$ th harmonic varies as the $q$ th power of the incident laser intensity, the harmonic intensity profile is given by

$$
\mathrm{I}_{q \omega} \sim \mathrm{I}_{1 \omega}^{q}=\mathrm{I}_{o}^{q} e^{-2 q r^{2} / w_{o}^{2}}
$$

where $w_{0}$ is the gaussian waist of the beam.[siegm86] In the far field $\left(z \gg z_{R}\right)$ the divergence of a gaussian beam is given by

$$
\theta_{\mathrm{div}} \simeq \frac{w(z)}{z} \simeq \frac{\lambda}{\pi w_{0}}
$$

Since the wavelength of the harmonic is given by $\lambda_{q}=\lambda_{1 \omega} / q$ the divergence angle for the qth harmonic would be

$$
\theta_{q} \simeq \frac{\lambda_{q}}{\pi w_{q}}=\frac{\lambda_{1 \omega}}{q \pi\left(w_{0} / q^{1 / 2}\right)}=\frac{\theta_{1 \omega}}{q^{1 / 2}}
$$



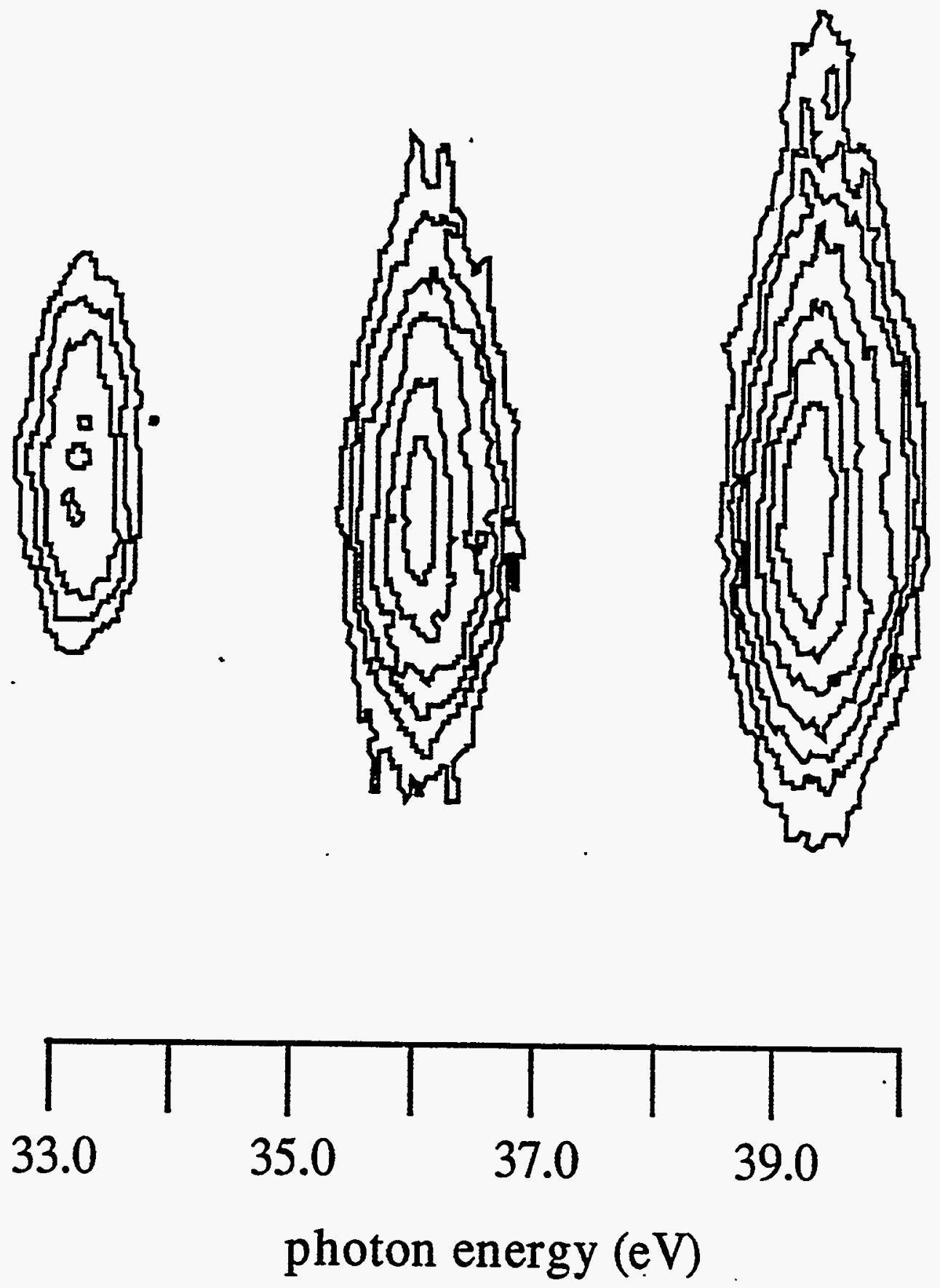

Figure 5.2: A contour plot of the 25th, 23rd and 21st harmonics produced in argon with the LiSAF laser at $1.2 \times 10^{14} \mathrm{~W} / \mathrm{cm}^{2}$. 

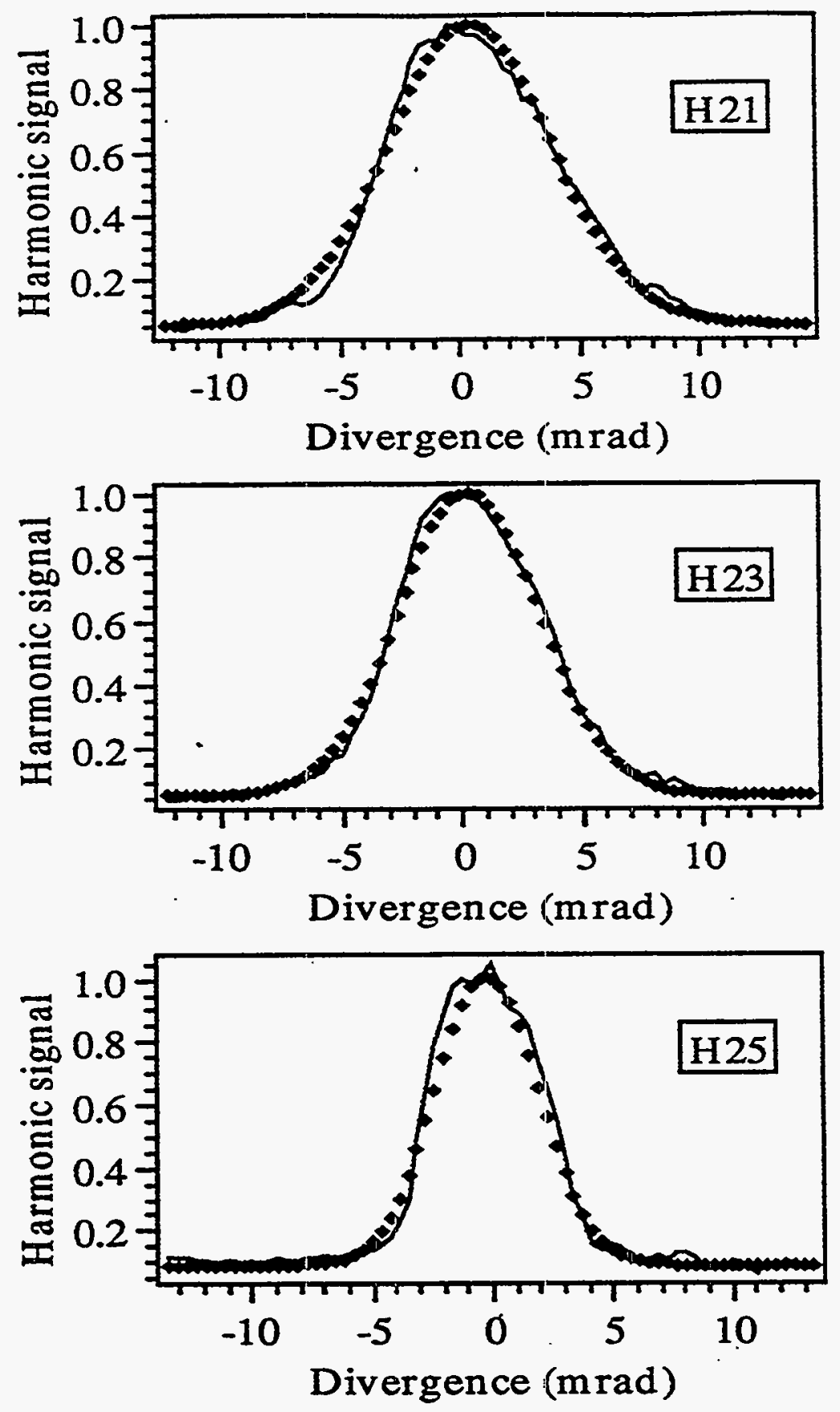

Figure 5.3: Integrated vertical lineouts for the 21st, 23rd, and 25th harmonics produced in argon with the LiSAF laser. The solid line is the experimentally measured spatial profile and the dotted line is a gaussian fit to the profile. 
However, it has already been established that the harmonic has associated with it an effective nonlinear order $p$ which would make the intensity of the $q$ th harmonic

$$
\mathrm{I}_{q \omega} \sim \mathrm{I}_{1 \omega}^{p}=\mathrm{I}_{o}^{p} e^{-2 p r^{2} / w_{0}^{2}} .
$$

Therefore, the divergence of the $q$ th harmonic would be given by

$$
\theta_{q} \simeq \frac{\lambda_{q}}{\pi w_{q}}=\frac{\lambda_{1 \omega}}{q \pi\left(w_{0} / p^{1 / 2}\right)}=\frac{p^{1 / 2} \theta_{1 \omega}}{q}
$$

For the twenty-fifth harmonic in argon the effective nonlinear order is $\sim 9$ yielding an expected divergence of $6.6 \mathrm{mrad}$, in good agreement with the experimental measurment. By this simple argument, the angular divergence of a given harmonic will depend strongly upon whether it is in the plateau or cutoff since the nonlinear order changes sharply between these two regions.

An image of the spatial profiles of the twenty-ninth through thirty-ninth harmonics obtained in neon with the LiSAF laser at an intensity of $2.5 \times 10^{14} \mathrm{~W} / \mathrm{cm}^{2}$ is shown in Figure 5.4. The results are very similar to those obtained in argon. The profiles are structureless and are well approximated by a gaussian fit. Lineouts of the spatial profiles of the twenty-ninth and thirty-ninth harmonics are shown in Figure 5.5 where the peak signal has been normalized to one. The full width of the profiles is narrowing as the wavelength gets shorter. The divergence predicted by the effective nonlinear order is $\sim 5 \mathrm{mrad}$ for the thirty-ninth harmonic which lies in the cutoff, $p \sim 13$, at this intensity. The predicted width for the twenty-ninth harmonic is $\sim 7 \mathrm{mrad}$ since is it positioned in the cutoff with a $p \sim 9$. These values are again in reasonable agreement with the experimental profiles.

An interesting feature was observed when the intensity was increased to above the saturation intensity for ionization of the target gas and is illustrated in Figure 5.6. The twenty-first harmonic produced in argon with the LiSAF laser is shown. The intensity was $8 \times 10^{14} \mathrm{~W} / \mathrm{cm}^{2}$, well above the saturation intensity of argon $\left(\mathrm{I}_{\text {sat }} \sim 3 \times 10^{14} \mathrm{~W} / \mathrm{cm}^{2}\right)$. The image is very distorted, exhibiting a crescent shape. The central portion of the harmonic ( $b$, on-axis with the laser-beam) exhibits a blue shift (shift toward shorter wavelength). Slightly off-axis $(\mathrm{a}, \mathrm{c})$ a high intensity feature 


\section{harmonic order}
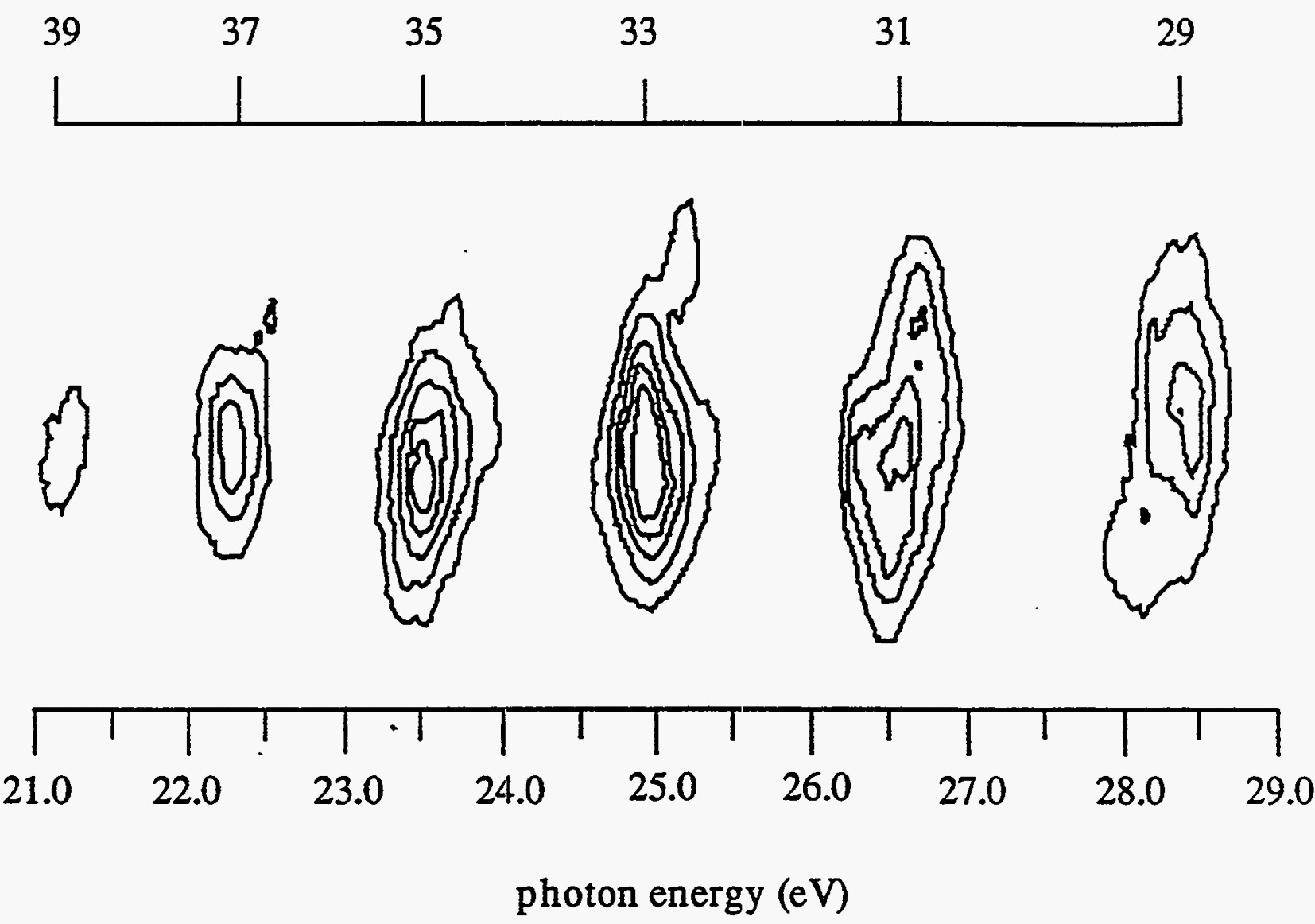

Figure 5.4: Spatial profiles of the 29th through 39th harmonics produced in neon with the LiSAF laser at an intensity of $2.5 \times 10^{14} \mathrm{~W} / \mathrm{cm}^{2}$.

is observed at the unshifted wavelength. This is consistent with previous experiments which reported blue-shifting of the harmonics as a direct result of the blue-shifting of the fundamental laser radiation in the plasma being formed.[1huil93b] The creation of free electrons in the focal volume causes a change in the index of refraction of the plasma which results in a shifting toward shorter wavelengths. The lower intensity portions of the laser profile are not ionizing the medium so do not see this effect.

In general, however, if the intensity of the incident laser is kept below the saturation intensity for ionization of the target gas then the spatial profiles of the harmonics 

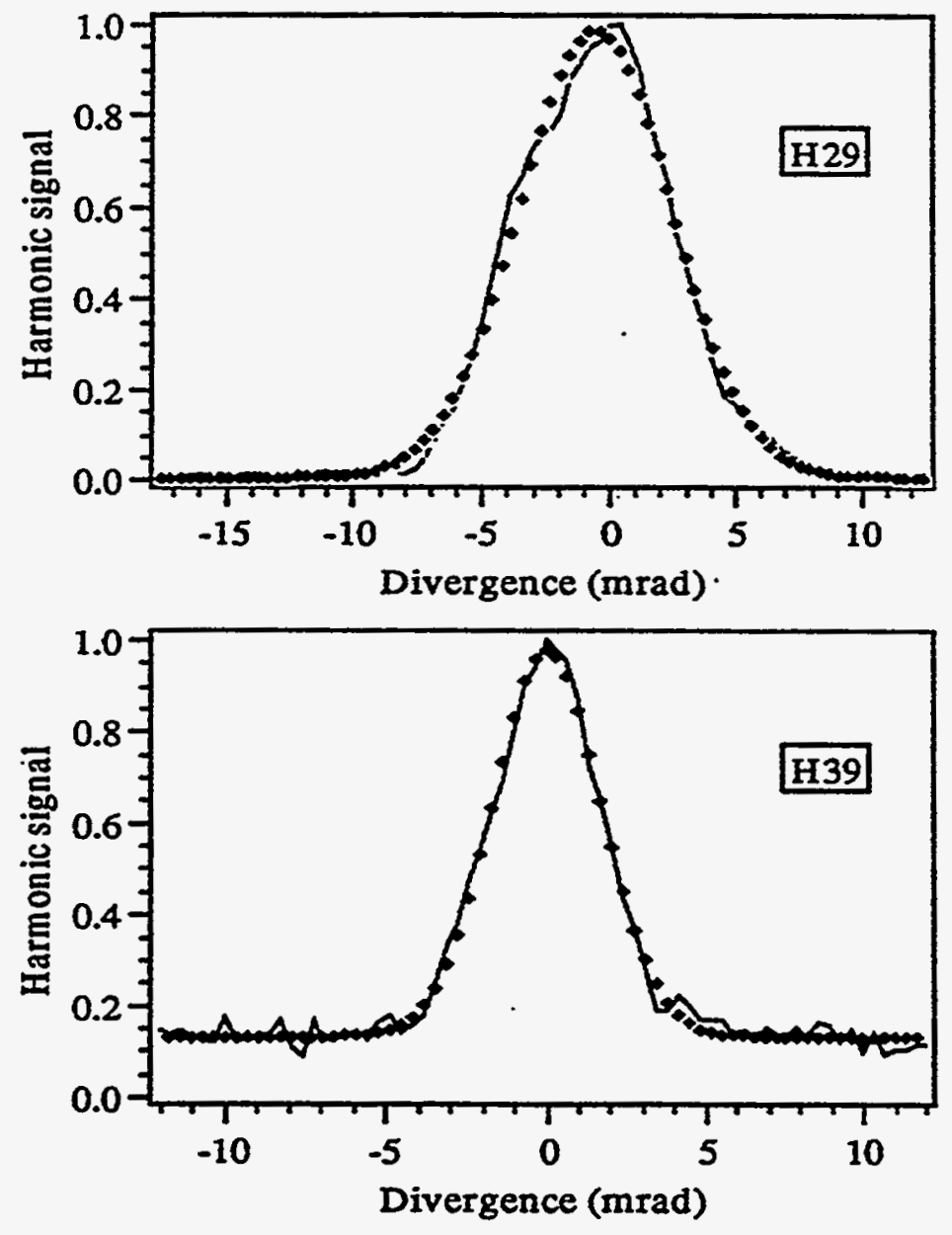

Figure 5.5: Spatial profiles of the 29th and 39th harmonics produced in neon. The solid line is the experimental profiles and the dotted line is a gaussian fit to the data. 


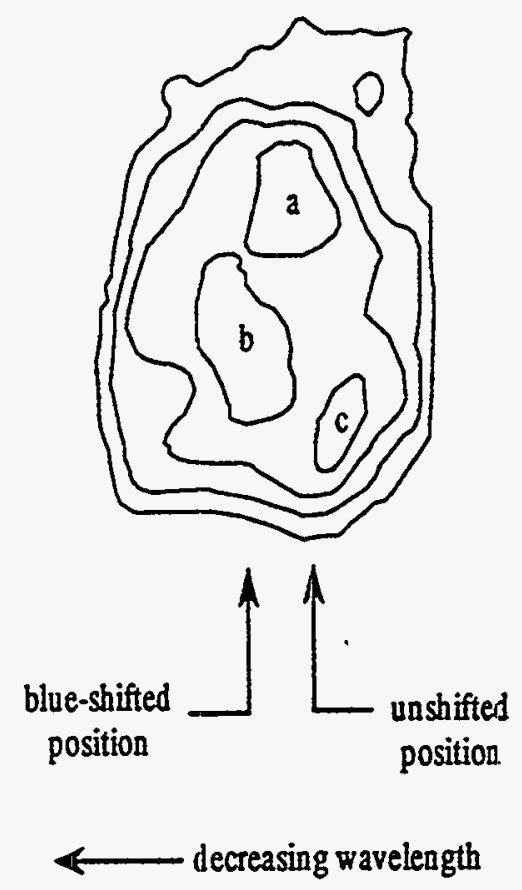

Figure 5.6: Contour plot of the spatial profile of the 21st harmonic produced in argon with the LiSAF laser at an intensity of $8 \times 10^{14} \mathrm{~W} / \mathrm{cm}^{2}$. The field-free and blueshifted positions of the harmonic are indicated. The contours labeled $a, b$, and $c$ are of equal intensity.

are well suited to secondary applications. They exhibit virtually no structure and their divergence is well-approximated by the divergence of a gaussian beam which would indicate that they can be refocused.

\subsection{Application of High Order Harmonic Gener- ation to Spectroscopy}

One of the goals of this research was to demonstrate the feasability of a high order harmonic "source" by examining the single-photon photoionization cross sections of the rare gases. Two primary features of this method-the high photon energy achievable and the ease of tunability of the source-were investigated.[balco93] The experimental apparatus is shown in Figure 5.7. The harmonic radiation is produced, as before, 


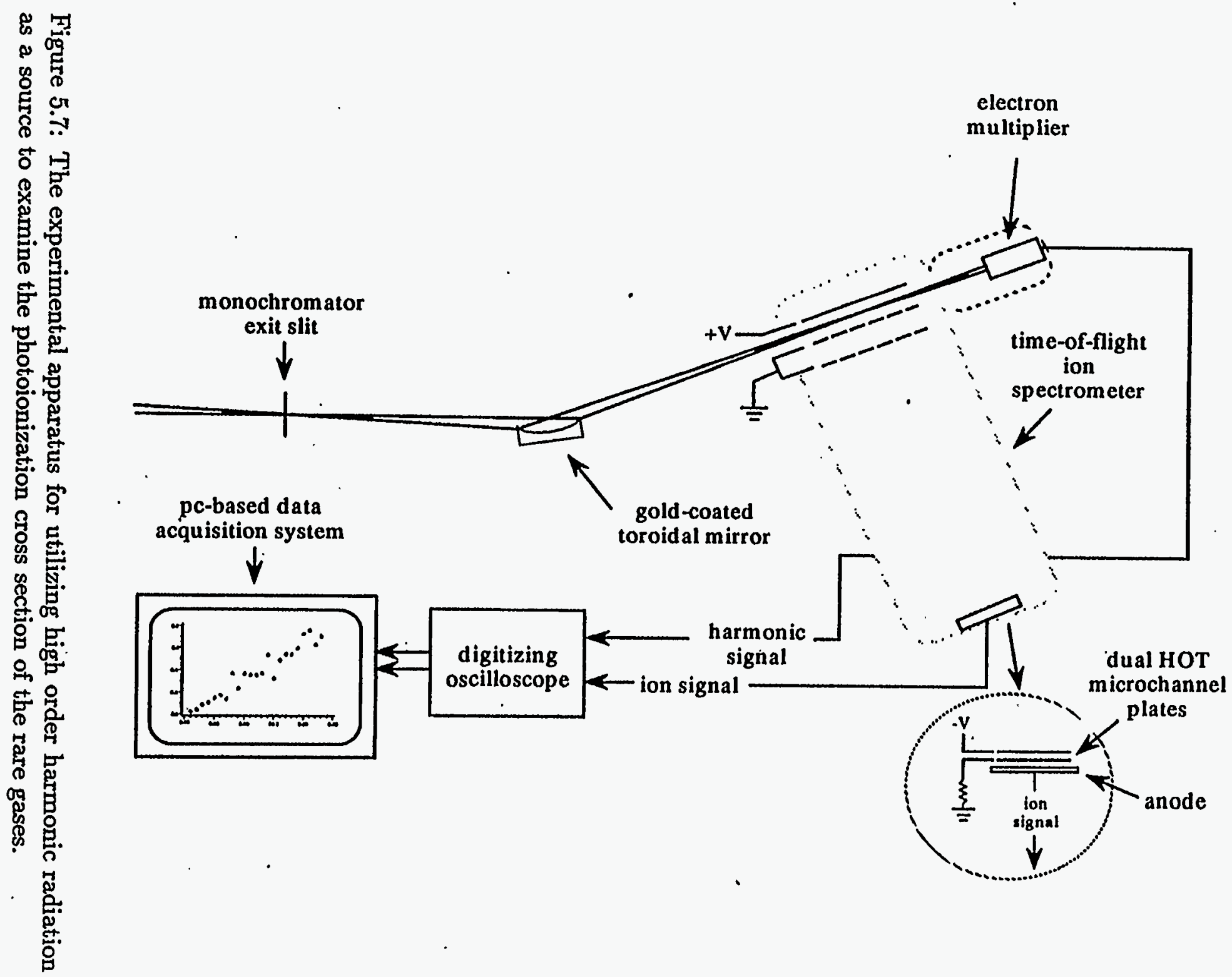


by focusing the incident laser radiation at the output of a pulsed gas jet. The harmonics produced are spectrally dispersed by a $1 \mathrm{~m}$ flat-field monochromator and an exit slit selects the wavelength to be utilized. The chosen harmonic is refocused by a $40 \mathrm{~cm}$ gold-coated toroidal mirror between the extraction grids of a time-of-flight ion spectrometer.

The target was provided by a second pulsed gas jet yielding atomic densities $\sim 10^{18}$ particles $/ \mathrm{cm}^{3}$ or a static pressure of gas which produced a target density of $10^{-4}$ Torr. Any ions that are produced are accelerated toward the flight tube by an extraction field, typically $300 \mathrm{~V} / \mathrm{cm}$, produced by a plate kept at a positive voltage, $600 \mathrm{~V}$, and a grounded wire grid separated by $2 \mathrm{~cm}$. The field-free region is $60 \mathrm{~cm}$ long with a microchannel plate detector at its end. The detector consists of two $4 \mathrm{~cm}$ diameter microchannel plates in chevron configuration with a $50 \Omega$ anode for detecting the electron current leaving the rear plate. Residual harmonic radiation passes through the interaction region onto an electron multiplier for detection. The outputs of the anode and electron multiplier are sent to a digitizing oscilloscope where they are integrated and collected by a PC-based data acquisition system which bins the number of ions produced with respect to the number of harmonic photons detected. The data are averaged over many shots per bin, typically twenty or more. By plotting the number of ions produced as a function of the incident harmonic flux the product $\int N \sigma_{a} d z$ may be extracted where $\sigma_{a}$ is the photoabsorption cross section. Figure 5.8 shows the number of ions produced in krypton as a function of the number of harmonic photons incident for the seventh harmonic of the dye laser operating at $604.5 \mathrm{~nm}$. The solid line is a least squares fit to the data, the slope of which yields the product mentioned above.

The first set of experiments performed utilized the picosecond dye laser system. Xenon was used as the generating medium due to its high efficiency. The photoionization of krypton was examined between 14 and $15 \mathrm{eV}$ by tuning the seventh harmonic of the incident laser by $.25 \mathrm{~nm}$ steps from 14.25 to $14.68 \mathrm{eV}$. The energy level structure of krypton in this region is shown in Figure 5.9. The ionization potentials of the ${ }^{2} P_{3 / 2}$ 


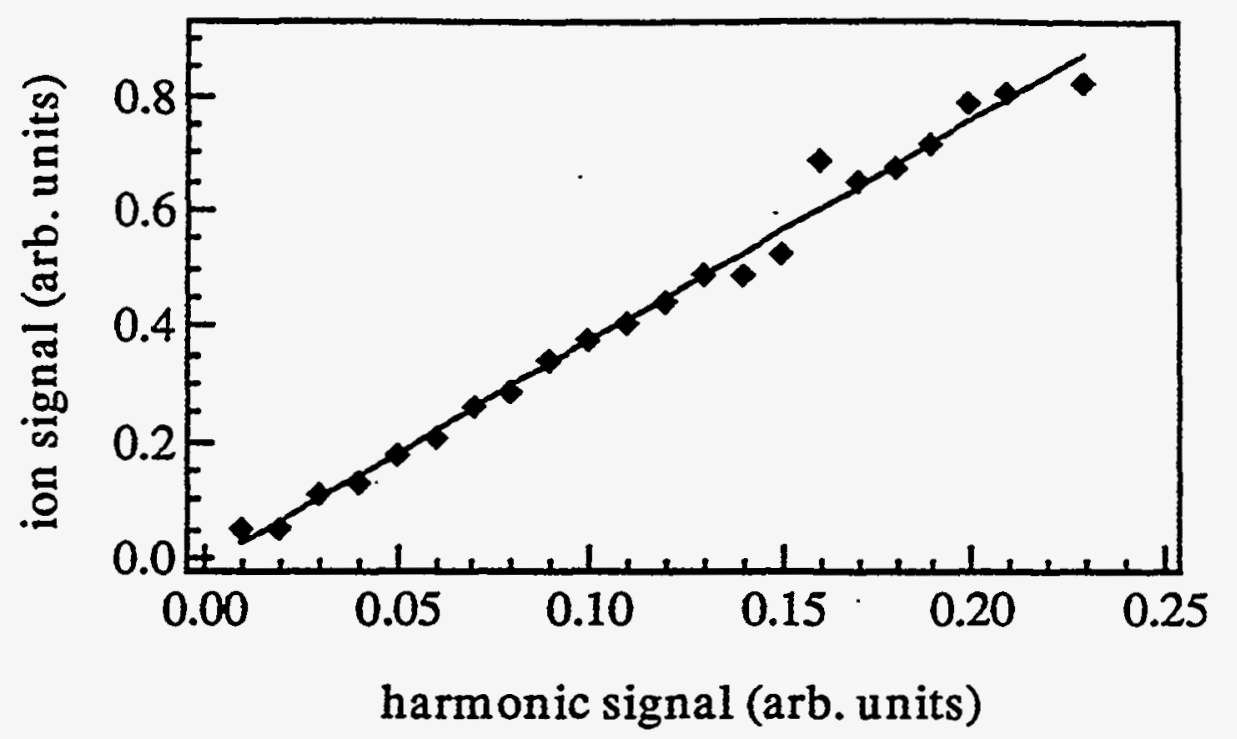

Figure 5.8: Number of ions produced in krypton as a function of number of harmonic photons produced by the seventh harmonic of the dye laser operating at $604.5 \mathrm{~nm}$. The solid line is a least squares fit to the data.

and ${ }^{2} P_{1 / 2}$ cores are $14.0^{\circ}$ and $14.67 \mathrm{eV}$ respectively and there are strong autoionizing resonances in the energy region between them. The data are shown in Figure 5.10 and characteristic Beutler-Fano profiles corresponding to $4 p^{6}-4 p^{5} n s^{\prime}$ and $4 p^{6}-4 p^{5} n d^{\prime}$ autoionizing transitions are observed.[chan92] For the dye laser system, the spectral linewidth has been calculated to be $0.014 \mathrm{~nm}$ at the seventh harmonic corresponding to a $\delta \nu / \nu=1.6 \times 10^{-4}$. The resolving power of the spectrometer, $\delta \nu / \nu=2 \times 10^{-3}$, is thus the limiting factor in the resolution of this scan.

The second experiment performed was to examine the photoionization cross section of the rare gases utilizing the Cr:LiSAF laser system and much higher order harmonic radiation. Figure 5.11 shows the absolute photoionization cross section of neon as a function of photon energy. The solid line represents the cross section measured by a high resolution dipole $(e, e)$ spectrometer [chan92b] and each experimental point is the value of the cross section for a specific harmonic. The agreement between this measurement and the previous measurement is quite good. This allows for a very accurate calibration of the spectrometer (via the exact wavelength of a given 


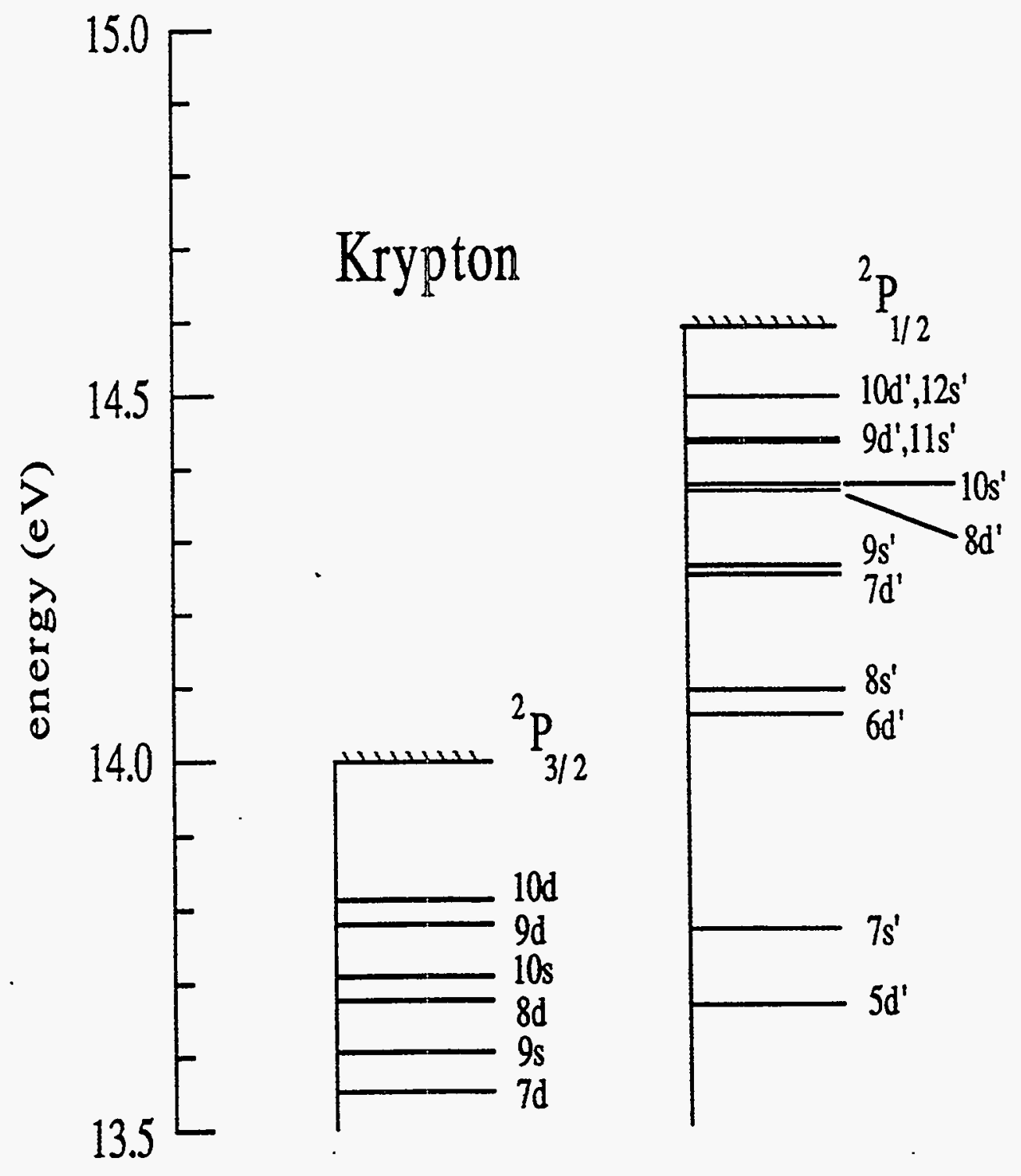

Figure 5.9: The energy level structure of krypton. 


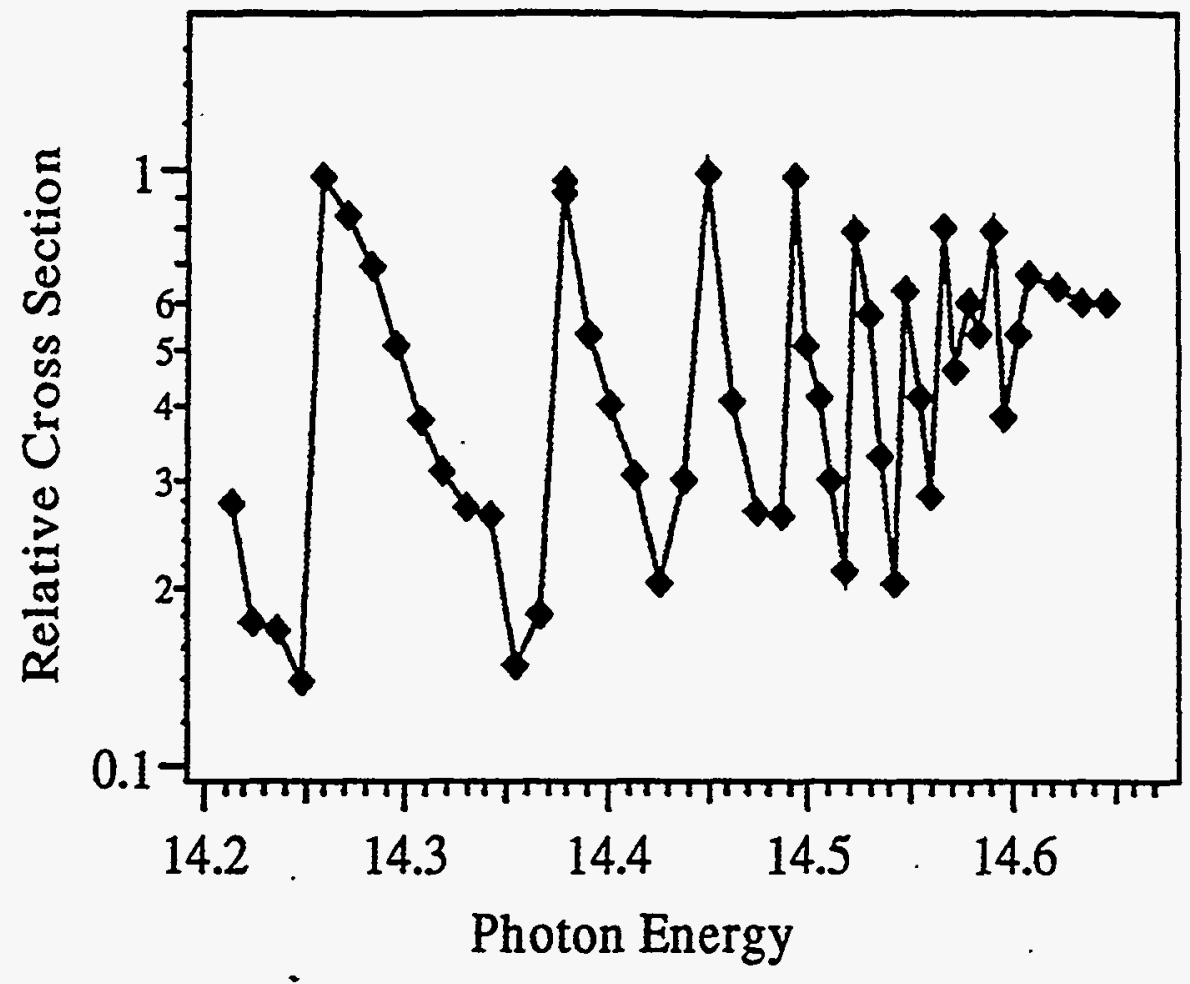

Figure 5.10: Relative photoionization cross section of krypton as a function of photon energy.

harmonic) as well as a direct measure of the number of harmonic photons produced.

There are several changes which could greatly improve the potential of this apparatus as a source of coherent XUV radiation. These experiments were performed at a 1-5 $\mathrm{Hz}$ repetition rate where the average power of the harmonic radiation is very low. However, by utilizing a higher repetition rate laser source, possibly $\geq 1 \mathrm{kHz}$, this limitation could be overcome. Better focusing optics could be used to focus the harmonics to a much smaller spot, thus increasing the intensity. A laser could be used as a high power pump beam with the harmonic beam acting as the low power probe for more sophisticated experiments and, in principle, the same laser could be used to generate the harmonics and to serve as the experimental pump. The many uses of this source are only beginning to be exploited. 


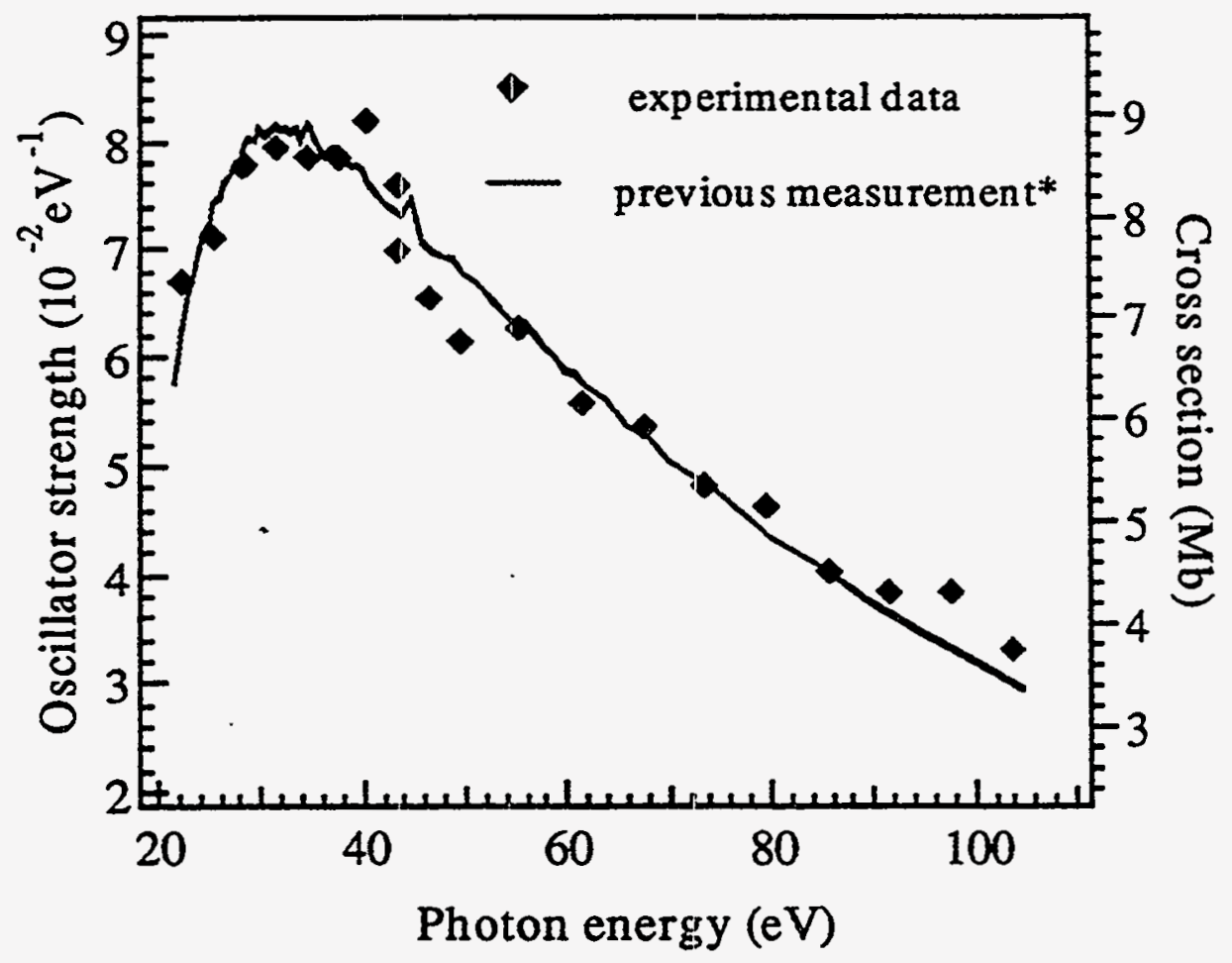

Figure 5.11: Absolute photoionization cross section of neon as a function of photon energy. The solid line is the cross section as measured by a dipole $(e, e)$ spectrometer. 


\section{Chapter 6}

\section{Summary}

The generation of short wavelength radiation has long been a topic of intense study. Recent experiments have demonstrated the production of harmonic radiation extending below $70 \AA$ [crane94] as well as conversion efficiencies in excess of $10^{-7}$ for harmonics extending to $200 \AA$ [ditmi94b]. Harmonic generation provides the possibility of a compact; coherent, tunable source of XUV radiation with a very high peak brightness. Two primary goals have been addressed herein. The first was to extend the understanding of the basic physics issues underlying the process of harmonic generation while the second focused on the development of this process as a usable "source".

\subsection{Conclusions}

Theory efforts based on the solution of the time-dependent Schrödinger equation have provided a tremendous amount of insight into the physics underlying harmonic generation. In an effort to provide a simple, intuitive model of the process which requires less computational sophistication, a semiclassical theory has been put forth which appears to capture the essence of harmonic generation while providing a very physical motivation for its conclusions. In this theory the atom first ionizes via tunneling and its subsequent motion in the laser field is treated classically. If during the course of its trajectory the electron reencounters the nucleus, it may recombine 
and emit a harmonic photon. Classically, the maximum energy an electron which is born at a phase where the ionization probability is significant may have when it passes the nucleus is $3.17 \mathrm{U}_{\mathrm{P}}$, thus recovering the phenomenological law for the position of the cutoff. A simple experimental test of this theory was done whereby the trajectory of the electron was perturbed by utilizing an elliptically polarized laser field. The twostep model predicts that increasing this ellipticity will very rapidly turn off harmonic generation and this effect was observed experimentally. The data were modeled using a simple quantum model and good agreement between the experiments and model calculations was observed.

The development of the harmonic "source" was the focus of the remaining experiments conducted. A systematic study of the spatial profiles of harmonic radiation was undertaken to investigate the potential of the source for applications requiring a high degree of spatial coherence. In contrast to earlier studies, the profiles were observed to be very smooth and structureless and to exhibit a divergence consistent with that expected from a calculation of the divergence of a gaussian beam with an effective nonlinear dependence of the driving field. The harmonics were then refocused into a secondary chamber containing a time-of-flight ion spectrometer and used to measure the photoabsorption cross section of several target species. The tunability of the source was verified by observing autoionizing resonances in krypton utilizing the seventh harmonic of the dye laser system produced in xenon. Harmonics with energies up to $103 \mathrm{eV}$ produced by the LiSAF laser system in neon were utilized to measure the photoabsorption cross section of neon. This measurement was in good agreement with previous measurements. These two experiments demonstrate the many exciting features which an XUV source based on high order harmonic generation can provide.

\subsection{Future Work}

The use of the harmonic source as a research tool in many areas of physics shows a great deal of promise. Applications in atomic and molecular physics, holography, 
microscopy, lithography, and $\mathrm{x}$-ray laser research are just a few of the possibilities. With laser technology continually moving forward new areas of research will almost certainly be added to this list. Currently, kilohertz repetition rate lasers offer the opportunity to extend this source to the regime of high average power applications, instead of those requiring only high peak power. Titanium sapphire lasers provide a good combination of high repetition rates, tunability, and moderate energy delivery and are becoming more and more common in laboratory settings.

A second extension of this work can be found in the two-step semiclassical model. This model has been extended to two dimensions to study the influence of elliptically polarized light on the production of harmonics. However, instead of having two perpendicular components of the same field, two fields of different frequencies, - polarized parallel or perpendicular to each other, could easily be studied by this method.[perry93] 


\section{Bibliography}

[agost79] P. Agostini, F. Fabre, G. Mainfray, G. Petite, and N. K. Rahman, "FreeFree Transitions Following Six-Photon Ionization of Xenon Atoms", Physical Review Letters 4:2, 1127-1130 (1979).

[ammos86] M. V. Ammosov, N. B. Delone, and V. P. Krainov, "Tunnel ionization of complex atoms and of atomic ions in an alternating electromagnetic field”, Soviet Physics JETP 64, 1191-1194 (1986).

[augst91] S. Augst, D. D. Meyerhofer, D. Strickland, and S. L. Chin, "Laser ionization of noble gases by Coulomb-barrier suppression", Journal of the Optical Society of America B 8, 858-867 (1991).

[balco93] P. Balcou, P. Salières, A. L'Huillier, K. S. Budil, T. Ditmire, and M. D. Perry, "The Application of High Order Harmonic Radiation to Photoionization Spectroscopy", submitted to Optics Letters.

[banda90] G. Bandarage, A. Maquet and J. Cooper, "Harmonic Generation by a Classical Hydrogen Atom in the Presence of an Intense Radiation Field", Physical Review A 41, 1744-1746 (1990).

[becke90] W. Becker, S. Long, and J. K. McIver, "Higher-harmonic production in a model atom with short-range potential" ${ }^{n}$, Physical Review $A$ 41, 41124115 (1990). 
[bethe77] H. A. Bethe and E. E. Salpeter, Quantum Mechanics of One- and TwoElectron Atoms, (Plenum, New York, 1977).

[biede89] L. C. Biedenharn, J. C. Solem, and G. A. Rinker, "Solvable Approximate Model for the Harmonic Radiation from Atoms Subjected to Oscillatory Electric Fields", Journal of the Optical Society of America B 6, 221-227 (1989).

[bjork75] G. C. Bjorklund, "Effects of focusing on third-order nonlinear processes in isotropic media", IEEE Journal of Quantum Electronics QE-11, 287-296 (1975).

[bokor83] J. Bokor, P. H. Bucksbaum, and R. R. Freeman, "Generation of $35.5 \mathrm{~nm}$ coherent radiation", Optics Letters 8, 217-219 (1983).

[budil93] K. S. Budil, P. Salières, A. L'Huillier, T. Ditmire, and M. D. Perry, "The Influence of Ellipticity on High Order Harmonic Generation", Physical Review A 48, R3437-R3440 (1993).

[chan92] W. F. Chan, G. Cooper, X. Guo, and C. E. Brion, "Absolute optical oscillator strengths for the electronic excitation of atoms at high resolution. III. The photoabsorption of argon, krypton, and xenon", Physical Review A 46, 149-171 (1992).

[chan92b] W. F. Chan, G. Cooper, X. Guo, and C. E. Brion, “Absolute optical oscillator strengths for the electronic excitation of atoms at high resolution. II. The photoabsorption of neon", Physical Review A 45, 1420-1433 (1992).

[chu90] S. I. Chu, K. Wang and E. Layton, "Nonperturbative Treatments of Level Shifts of Excited States and High Order Harmonic Generation in Strong Fields", Journal of the Optical Society of America B 7, 425-432 (1990). 
[corku93] P. B. Corkum, "Plasma Perspective on Strong-Field Multiphoton Ionization”, Physical Review Letters 71, 1994-1997 (1993).

[crane92] J. K. Crane, M. D. Perry, S. Herman, and R. W. Falcone, "High Field Harmonic Generation in Helium", Optics Letters 17, 1256-1258 (1992).

[crane94] J. K. Crane, H. Nguyen, and M. D. Perry, to be published.

[ditmi93] T. Ditmire and M. D. Perry, "Terawatt $\mathrm{Cr}: \mathrm{LiSrAlF}_{6}$ laser system", Optics Letters 18, 426-428 (1993).

[ditmi94a] T. Ditmire, H. Nguyen, and M. D. Perry, "Design and Performance of Multi-Terawatt $\mathrm{Cr}: \mathrm{LiSrAlF}_{6}$ Laser System", Journal of the Optical Society of America B 11, 580-590 (1994:).

[ditmi94b] T. Ditmire, K. S. Budil, J. K. Crane, H. Nguyen, and M. D. Perry, "Coherence properties of high order harmonic radiation", in the proceedings of Ultrafast Phenomena-Ninth International Meeting, May 1-5, 1994, Dana Point, California.

[eber189] J. H. Eberly, K. C. Kulander, Q. Su, B. W. Shore, J. Javanainen and L. Roso-Franco, "High-order harmonic generation during multiphoton ionization of gases", Journal of Modern Optics 36, 829-855 (1989).

[eber189b] J. H. Eberly, Q. Su, and J. Javanainen, "Nonlinear light scattering accompanying multiphoton ionization", Physical Review Letters 62, 881-884 (1989).

[egger80] H. Egger, R. T. Hawkins, J. Bokor, H. Pummer, M. Rothschild, and C. K. Rhodes, "Generation of high-spectral-brightness tunable XUV radiation at $83 \mathrm{~nm}^{\prime}$, Optics Letters 5, 282-284 (1980).

[ferra88] M. Ferray, A. L'Huillier, X. F. Li, L. A. Lompré, G. Mainfray, and C. Manus, "Multiple harmonic conversion of $1064 \mathrm{~nm}$ radiation in rare gases", Journal of Physics B 21, L31-L35 (1988). 
[frank61] P. A. Franken, A. E. Hill, C. W. Peters, and G.Weinreich, "Generation of optical harmonics", Physical Review Letters 7, 118-119 (1961).

[frant63] L. M. Frantz and J. S. Nodvik, "Theory of pulse propagation in a laser amplifier", Journal of Applied Physics 34, 2346-2349 (1963).

[goppe31] M. Göppert-Mayer, "Two Quantum Processes", Annalen der Physik 9, 273-294 (1931).

[haigh93] R. Haight and D. R. Peale, "Antibonding State on the Ge(111)-As Surface-Spectroscopy and Dynamics”, Physical Review Letters 70, 39793982 (1993).

[jacks75] J. D. Jackson, Classical Electrodynamics, (John Wiley \& Sons, New York, 1975).

[kaise61] W. Kaiser and C. G. B. Garrett, "Two-photon excitation in $\mathrm{CaF}_{2} \mathrm{Eu}^{2+»}$, Physical Review Letters 7, 229-231 (1961).

[keldy65] L. V. Keldysh, "Ionization in the Field of a Strong Electromagnetic Wave", Soviet Physics JETP 20, 1307-1314 (1965).

[klein62] D. A. Kleinman, "Theory of second harmonic generation of light", Physical Review 128, 1761-1775 (1987).

[kraus92] J. L. Krause, K. J. Schafer, and K. C. Kulander, "Calculation of photoemission from atoms subject to intense laser fields", Physical Review $A$ 45, 4998-5010 (1992).

[kraus92b] J. L. Krause, K. J. Schafer and K. C. Kulander, "High-order harmonic generation from atoms and ions in the high intensity regime", Physical Review Letters 68, 3535-3538 (1992). 
[kruit81] P. Kruit, J. Kimman, H. G. Muller, and M. J. van der Wiel, "Absorption of additional photons in the multiphoton ionisation continuum of xenon

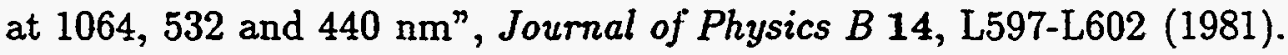

[kulan89] K. C. Kulander, and B. W. Shore, "Calculations of multiple-harmonic conversion of $1064 \mathrm{~nm}$ radiation in Xe", Physical Review Letters 62, 524526 (1989).

[kulan93] K. C. Kulander, K. J. Schafer, and J. L. Krause, Proceedings of the Workshop SILAP III, (Plenum Press, New York), B. Piraux editor, in press.

[lhuil91] A. L'Huillier, K. J. Schafer, and K. C. Kulander, "High-order harmonic generation in xenon at $1064 \mathrm{~nm}$ : the role of phase matching", Physical Review Letters 66, 2200-2203 (1991).

[lhuil92] Anne L'Huillier, Philippe Balcou, and L. A. Lompré, "Coherence and Resonance Effects in High-Order Harmonic Generation", Physical Review Letters 68, 166-169 (1992).

[lhuil93] A. L'Huillier and Ph. Balcou, "High-Order Harmonic Generation in Rare Gases with a 1 ps 1053 nm Laser", Physical Review Letters 70, 774-777 (1993).

[lhuil93b] A. L'Huillier, M. Lewenstein, P. Saliéres, $\mathrm{Ph}$. Balcou, J. Larsson, and C. G. Wahlström, "High-order harmonic generation cutoff", Physical Review A 48, R3433-R3436 (1993).

[lewen94] M. Lewenstein, Ph. Balcou, M. Yu. Ivanov, A. L'Huillier, and P. B. Corkum, "Theory of high harmonic generation by. low frequency laser fields", Physical Review A 49, 2117-2132 (1994).

[lewen94b] M. Lewenstein and A. L'Huillier, private communication. 
[li89] X. F. Li, A. L'Huillier, M. Ferray, L. A. Lompré, and G. Mainfray, "Multiple-harmonic generation in rare gases at high laser intensity", Physical Review A 39, 5751-5761 (1989).

[liang94] Y. Liang, M. V. Ammosov, and S. L. Chin, "High-order Harmonic Generation in Argon by Elliptically Polarized Picosecond Dye Laser Pulses", Journal of Physics B 27, 1269-1276 (1994).

[lompr88] L. A. Lompré, M. Ferray, A. L'Huillier, X. F. Li, and G. Mainfray, "Optical determination of the characteristics of a pulsed-gas jet", Journal of Applied Physics 63, 1791-1793 (1988).

[mack193] J. J. Macklin, J. D. Kmetec, and C. L. Gordon III, "High-Order Harmonic Generation Using Intense Femtosecond Pulses", Physical Review Letters 70, 766-769 (1993).

[maine88] P. Maine, D:Strickland, P. Bado, M. Pessot, and G. Mourou, "Generation of Ultrahigh Peak Power Pulses by Chirped Pulse Amplification", IEEE Journal of Quantum Electronics QE-24, 398-403 (1988).

[marti87] O. E. Martinez, "3000 Times Grating Compressor with Positive Group Velocity Dispersion: Application to Fiber Compensation in the 1.3-1.6 $\mu \mathrm{m}$ Region", IEEE Journal of Quantum Electronics QE-23; 59-64 (1987).

[mcphe87] A. McPherson, G. Gibson, H. Jara, U. Johann, T. S. Luk, I. A. McIntyre, K. Boyer, and C. K. Rhodes, "Studies of multiphoton production of vacuum-ultraviolet radiation in the rare gases", Journal of the Optical Society of America B 4, 595-601 (1987).

[miyaz89] K. Miyazaki, H. Sakai, and T. Sato, "Two-photon resonances in Xe and $\mathrm{Kr}$ for the generation of tunable coherent extreme UV radiation", Applied Optics 28, 699-702 (1989). 
[miyaz92] K. Miyazaki and H. Sakai, "High order harmonic generation in rare gases with intense subpicosecond dye laser pulses", Journal of Physics B 25, L83-L89 (1992).

[manak80] N. L. Manakov and V. D. Ovsyannikov, "Higher-order nonlinear susceptibilities for generation of optical radiation harmonics in atomic gases", Soviet Physics JETP 52, 895-900 (1980).

[ng91] C. Y. Ng, Vacuum ultraviolet photoionization and photodissociation of molecules and clusters, (World Scientific, Singapore,1991).

[orr71] B. J. Orr and J. F. Ward, "Perturbation theory of the non-linear optical polarization of an isolated system", Molecular Physics 20, 513-526 (1971).

[page88] R. H. Page, R. J. Larkin, Y. R. Shen, and Y. T. Lee, "High-Resolution Photoionization Spectrum of Water Molecules in a Supersonic Beam", Journal of Chemical Physics 88, 2249-2263 (1988).

[pax88] P. Pax and J. Weston, "Novel large mode volume resonator", IEEE Journal of Quantum Electronics 27, 1242-1246 (1991).

[payne89] S. A. Payne, L. L. Chase, L. K. Smith, W. L. Kway, and H. W. Newkirk, "Laser Performance of LiSrAlF 6 : $\mathrm{Cr}^{3+»}$, Journal of Applied Physics 66, 1051-1056 (1989).

[peatr93] J. Peatross and D. D. Meyerhofer, "Measurement of the angular distributions of high-order harmonics", in Short Wavelength V: Physics with Intense Laser Pulses, M. D. Perry and P. B. Corkum, editors, Optical Society of America (1993).

[perry88] M. D. Perry, A. Szöke, O. L. Landen, and E. M. Campbell, "Nonresonant multiphoton ionization of the noble gases: theory and experiment", Physical Review Letters 60, 1270-1273 (1988). 
[perry89] M. D. Perry, O. L. Landen, J. Weston, and R. Ettelbrick, "Design and performance of a high-power, synchronized Nd:YAG-dye laser system", Optics Letters 14, $42-44$ (1989).

[perry92] M. D. Perry, D. Strickland, T. Ditmire, and F. G. Patterson, “Cr:LiSrAlF 6 regenerative amplifier”, Optics Letters 17, 604-606 (1992).

[perry92b] M. D. Perry, C. Darrow, C. Coverdale, and J. K. Crane, "Measurement of the local electron density by means of stimulated Raman scattering in a laser-produced gas jet plasma", Optics Letters 17, 523-525 (1992).

[perry93] M. D. Perry and J. K. Crane, "High-order harmonic emission from mixed fields", Physical Review A 48, R4051-R4054 (1993).

[potv189] R. M. Potvliege and R. Shakeshaft, "Multiphoton Processes in an Intense Laser Field-Harmonic Generation and Total Ionization Rates for Atomic Hydrogen", Physical Review A 40, 3061-3079 (1989).

[reint78] J. Reintjes and C. Y. She, "Comparison of fifth and third harmonic conversion in helium", Optics Communications 27, 469-474 (1978).

[reint84] J. F. Reintjes, Nonlinear Optical Parametric Processes in Liquids and Gases, (Orlando, Academic Press, Inc., 1984).

[salie94] P. Salières, A. L'Huillier, T. Ditmire, K. S. Budil, and M. D. Perry, "Spatial Profiles of High Order Harmonics Generated by a Short Pulse Cr:LiSAF laser", Journal of Physics B.

[saruk91] N. Sarukura, K. Hata, T. Adachi, R. Nodomi, M. Watanabe, and S. Watanabe, "Coherent Soft-X-Ray Generation by the Harmonics of an Ultrahigh-Power KrF Laser”, Physical Review A 43, 1669-1672 (1991). 
[schaf93] K. J. Schafer and K. C. Kulander, "Quantum and Quasi-Classical Analyses of High Order Emission Processes", in OSA Proceedings on Shortwavelength V: Physics with Intense Laser Pulses ('93), P. B. Corkum and M. D. Perry editors, (1993).

[shore87] B. W. Shore and P. L. Knight, "Enhancement of high optical harmonics by excess-photon ionization", Journal of Physics B 20, 413-423 (1987).

[siegm86] A. E. Siegman, Lasers, (Mill Valley, University Science Books, 1986).

[sizer81] T. Sizer, II, J. D. Kafka, A. Krisiloff, and G. Mourou, "Generation and amplification of sub-picosecond pulses using a frequency-doubled neodymium YAG pumping source”, Optics Communications 39, 259-262 (1981).

[stric85] D. Strickland and G. Mourou, "Compression of Amplified Chirped Optical Pulses", Optics Communications 56, 219-221 (1985).

[sunda90] B. Sundaram and P. W. Milloni, "High-Order Harmonic-GenerationSimplified Model and Relevance of Single-Atom Theories to Experiment", Physical Review A 41, 6571-6573 (1990).

[tisch94] J. W. G. Tisch, R. A. Smith, J. E. Muffett, M. Ciarrocca, J. P. Marangos, and M. H. R. Hutchinson, "Angularly resolved high-order harmonic generation in helium", Physical Review A 49, R28-R31 (1994).

[ward69] J. F. Ward and G. H. C. New, "Optical third harmonic generation in gases by a focused laser beam", Physical Review 185, 57-72 (1969).

[wokau82] A. Wokaun, P. F. Liao, R. R. Freeman, and R. H. Storz, "High-energy picosecond pulses: design of a dye-laser-amplifier system", Optics Letters 7, 13-15 (1982). 
[wyatt81] R. Wyatt and E. E. Marinero, "Versatile Single-Shot Background-Free Pulse Duration Measurement Technique, for Pulses of Subnanosecond to Picosecond Duration", Applied Physics 25, 297-301 (1981). 